\title{
Linear Perturbations of Quaternionic Metrics
}

\author{
Sergei Alexandrov ${ }^{1}$, Boris Pioline ${ }^{2,3}$, Frank Saueressig ${ }^{4}$, \\ Stefan Vandoren ${ }^{5}$ \\ ${ }^{1}$ Laboratoire de Physique Théorique \& Astroparticules ${ }^{\star}$, \\ Université Montpellier II, 34095 Montpellier Cedex 05, France. \\ E-mail: alexandrov@1pta.univ-montp2.fr \\ 2 Laboratoire de Physique Théorique et Hautes Energies ${ }^{\star \star}$, \\ Université Pierre et Marie Curie, 4 place Jussieu, 75252 Paris cedex 05, France. \\ E-mail: pioline@1pthe.jussieu.fr \\ 3 Laboratoire de Physique Théorique de l'Ecole Normale Supérieure ${ }^{\star \star \star}$, \\ 24 rue Lhomond, 75231 Paris cedex 05, France \\ ${ }^{4}$ Institut de Physique Théorique ${ }^{\star \star \star}$, CEA, F-91191 Gif-sur-Yvette, France. \\ E-mail: frank.saueressig@cea.fr \\ 5 Institute for Theoretical Physics and Spinoza Institute, \\ Utrecht University, Leuvenlaan 4, 3508 TD Utrecht, The Netherlands. \\ E-mail: S.J.G.Vandoren@uu.nl
}

Received: 25 November 2008 / Accepted: 18 December 2009

Published online: 25 February 2010 - (C) The Author(s) 2010. This article is published with open access at Springerlink.com

\begin{abstract}
We extend the twistor methods developed in our earlier work on linear deformations of hyperkähler manifolds [1] to the case of quaternionic-Kähler manifolds. Via Swann's construction, deformations of a $4 d$-dimensional quaternionic-Kähler manifold $\mathcal{M}$ are in one-to-one correspondence with deformations of its $4 d+4$-dimensional hyperkähler cone $\mathcal{S}$. The latter can be encoded in variations of the complex symplectomorphisms which relate different locally flat patches of the twistor space $\mathcal{Z}_{\mathcal{S}}$, with a suitable homogeneity condition that ensures that the hyperkähler cone property is preserved. Equivalently, we show that the deformations of $\mathcal{M}$ can be encoded in variations of the complex contact transformations which relate different locally flat patches of the twistor space $\mathcal{Z}_{\mathcal{M}}$ of $\mathcal{M}$, by-passing the Swann bundle and its twistor space. We specialize these general results to the case of quaternionic-Kähler metrics with $d+1$ commuting isometries, obtainable by the Legendre transform method, and linear deformations thereof. We illustrate our methods for the hypermultiplet moduli space in string theory compactifications at tree- and one-loop level.
\end{abstract}

\section{Contents}

1. Introduction . . . . . . . . . . . . . . . . . . . . . . 354

2. Quaternionic-Kähler Geometry and Twistors . . . . . . . . . . . . . . . . . . . . . 357

2.1 Bottom-up: from QK to HKC . . . . . . . . . . . . . . . . . . . . . . . . . . . . . 357

2.2 Top down: from $\mathrm{HKC}$ to QK . . . . . . . . . . . . . . . . . 360

2.3 Patchwork construction of twistor spaces of HK manifolds - a summary 361

2.4 Conditions for superconformal invariance . . . . . . . . . . . . 363

* Unité mixte de recherche du CNRS UMR 5207.

$\star$ Unité mixte de recherche du CNRS UMR 7589.

$\star \star \star$ Unité mixte de recherche du CNRS UMR 8549.

$\star \star \star \star$ Unité de recherche associée au CNRS URA 2306. 
2.5 Homogeneous symplectic vs. contact geometry . . . . . . . . . . . 367

3. Quaternionic Geometry with Commuting Isometries . . . . . . . . . . . . 371

3.1 Tri-holomorphic isometries and superconformal invariance . . . . . . 371

3.2 Superconformal quotient . . . . . . . . . . . . . . . . 376

3.3 Contact twistor lines . . . . . . . . . . . . . . . . . . 378

4. The Perturbative Hypermultiplet Moduli Space . . . . . . . . . . . . . . . . 380

4.1 Tree-level geometry . . . . . . . . . . . . . . . . . . . . . . 380

4.2 One-loop correction . . . . . . . . . . . . . . . . . . 383

4.3 Superconformal quotient . . . . . . . . . . . . . . . . . . . 384

5. Linear Deformations of $\mathcal{O}(2)$ Quaternionic-Kähler Spaces . . . . . . . . . . 386

5.1 Linear deformations of $\mathcal{O}(2)$ hyperkähler cones . . . . . . . . . . . . . 386

5.2 Perturbed contact twistor lines . . . . . . . . . . . . . . . . . . . . . 389

A. Infinitesimal $S U(2)$ Transformations . . . . . . . . . . . . . . . . . 394

B. An alternative Formulation for Hypermultiplet Moduli Spaces . . . . . . . . 395

C. Deformed Superconformal Quotient . . . . . . . . . . . . . . . . . . . . . . 397

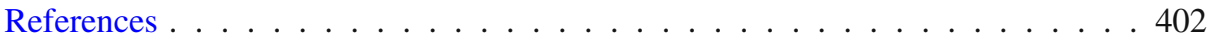

\section{Introduction}

Quaternionic-Kähler (QK) manifolds play an important role in string and supergravity theories, primarily because the hypermultiplet moduli spaces appearing in string theory backgrounds with 8 supercharges fall into this class. In this work, we study general aspects of QK manifolds and of their twistor spaces, and provide a general formalism for describing linear perturbations of $4 d$-dimensional QK manifolds with $d+1$ commuting isometries. For this purpose we build on our previous study of linear deformations of hyperkähler (HK) manifolds obtainable by the Legendre transform method [1].

A key fact for the present study is the (local) one-to-one correspondence between $4 d$-dimensional QK manifolds $\mathcal{M}$ and $4 d+4$-dimensional "hyperkähler cones" (HKC) $\mathcal{S}$, i.e. $4 d+4$-dimensional HK manifolds with a homothetic Killing vector and an isometric $S U$ (2) action rotating the three complex structures (see Fig. 1 for orientation). In particular, Swann's construction produces $\mathcal{S}$ as a $\mathbb{C}^{2} / \mathbb{Z}^{2}$ bundle over $\mathcal{M}$, twisted by the $S U$ (2) part of the spin connection on $\mathcal{M}$ [2]. The converse relation goes under the name of "superconformal quotient" in the physics literature [3,4]. Moreover, any isometry of $\mathcal{M}$ can be lifted to a tri-holomorphic isometry of $\mathcal{S}$, see e.g. [5,6]. Therefore, the formalism of [1] is directly applicable to the Swann bundle $\mathcal{S}$, with a suitable restriction to ensure the hyperkähler cone (or "superconformal invariance") property.

For this purpose, one introduces the twistor space $\mathcal{Z}_{\mathcal{S}}=\mathcal{S} \times \mathbb{C} P^{1}$ of the HK manifold $\mathcal{S}$, an open covering $\hat{\mathcal{U}}_{i}$ of $\mathcal{Z}_{\mathcal{S}}$ projecting to open disks $\mathcal{U}_{i}$ on $\mathbb{C} P^{1}$, and a local Darboux coordinate system $\left(v_{[i]}^{I}, \mu_{I}^{[i]}\right)(I=b, 0, \ldots, d-1)$ for the $\mathcal{O}(2)$-twisted complex symplectic structure $\Omega^{[i]}=\mathrm{d} \mu_{I}^{[i]} \wedge \mathrm{d} \nu_{[i]}^{I}$ on $\hat{\mathcal{U}}_{i}$. Since ${ }^{1} \Omega^{[i]}=f_{i j}^{2} \Omega^{[j]} \bmod \mathrm{d} \zeta$ on the overlap $\hat{\mathcal{U}}_{i} \cap \hat{\mathcal{U}}_{j}$, the coordinate systems $\left(v_{[i]}^{I}, \mu_{I}^{[i]}\right)$ and $\left(v_{[j]}^{I}, \mu_{I}^{[j]}\right)$ must be related by a symplectomorphism on $\hat{\mathcal{U}}_{i} \cap \hat{\mathcal{U}}_{j}$; the latter can be parametrized in the usual way by a generating function $S^{[i j]}\left(v_{[i]}^{I}, \mu_{I}^{[j]}, \zeta\right)$. The set of all $S^{[i j]}$, subject to consistency relations, reality conditions and gauge equivalence, encodes the complex symplectic structure on $\mathcal{Z}_{\mathcal{S}}$, and therefore the $\mathrm{HK}$ metric on $\mathcal{S}$.

\footnotetext{
${ }^{1}$ Here $f_{i j}$ are the transition functions of the $\mathcal{O}(1)$ bundle on $\mathbb{C} P^{1}$ with coordinate $\zeta$.
} 


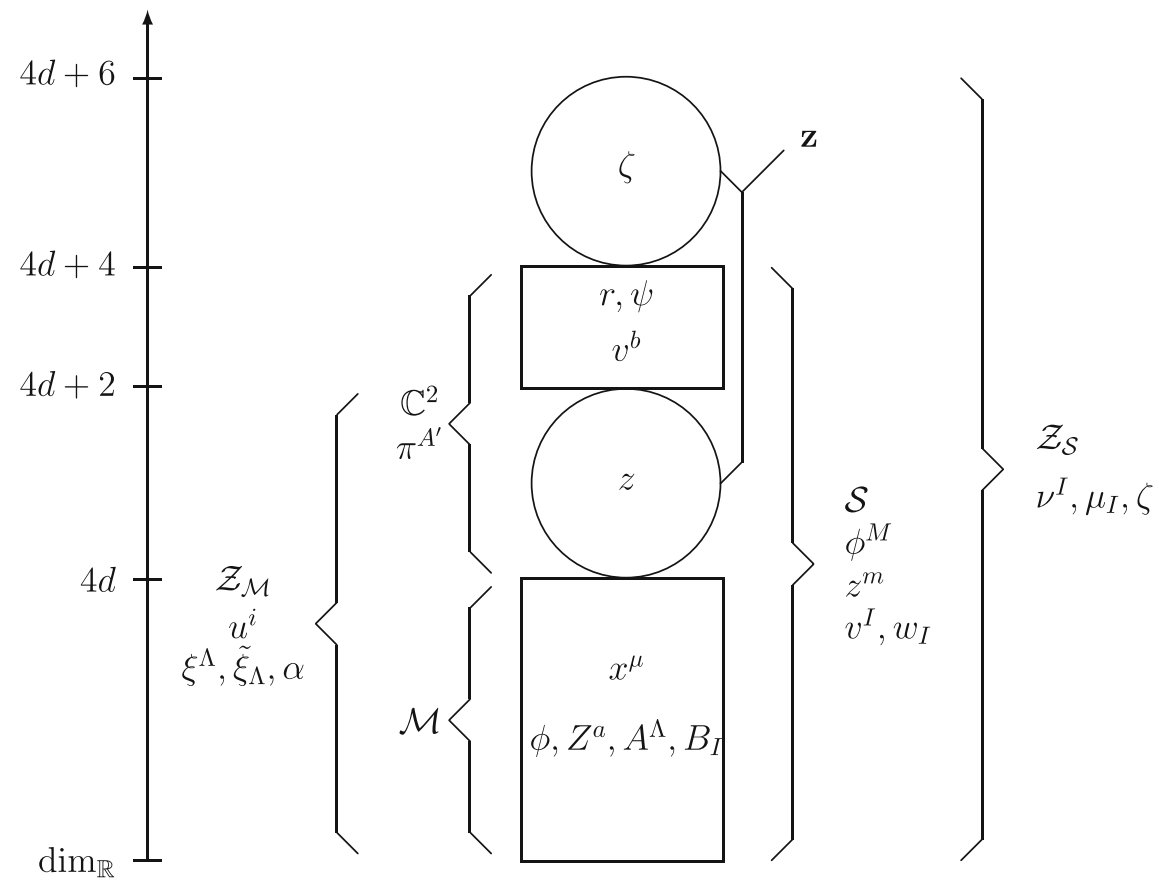

Fig. 1. Summary of various coordinate systems on the $\mathrm{QK}$ space $\mathcal{M}$, its twistor space $\mathcal{Z}_{\mathcal{M}}$, its Swann bundle $\mathcal{S}$ and the twistor space of the Swann bundle $\mathcal{Z}_{\mathcal{S}}$

As we show in Sect. 2.4 below, superconformal invariance restricts $S^{[i j]}$ to be a function of $f_{i j}^{-2 n} \nu_{[i]}^{I}$ and $\mu_{I}^{[i]}$ only, with no further $\zeta$ dependence, and to be homogeneous of degree one when $\left(v_{[i]}^{I}, \mu_{I}^{[i]}\right)$ are rescaled with weight $(n, 1-n)$, respectively ${ }^{2}$. The integer $n$ characterizes the transformation rules of the local coordinates under both dilations and $S U$ (2) rotations. For $n=1$, the relevant case for QK manifolds with isometries, the $\mathcal{O}(0)$ sections $\mu_{I}^{[i]}$ may acquire anomalous scaling dimensions, and the homogeneity condition may be relaxed into a "quasi-homogeneity" property, as explained further in Sect. 2.4.

Deformations of $\mathcal{S}$ correspond to variations $H_{(1)}^{[i j]}\left(v_{[i]}^{I}, \mu_{I}^{[i]}, \zeta\right)$ of the generating functions $S^{[i j]}$, subject to the consistency, reality and quasi-homogeneity conditions and gauge equivalence. When $\mathcal{S}$ is obtainable by the Legendre transform method, which is the case when $\mathcal{M}$ admits $d+1$ commuting isometries, the deformed twistor lines and hyperkähler potential are easily computed to first order in the perturbation. The deformed QK metric may in principle be obtained by the standard superconformal quotient procedure. In Appendix C, we construct a natural set of coordinates on the deformed QK manifold, but stop short of writing the deformed metric explicitly, as the expressions would be too cumbersome.

While the strategy outlined above is conceptually straightforward, it is rather unpractical. As we explain in detail in Sect. 2.5, one may by-pass the twistor space of the Swann bundle $\mathcal{Z}_{\mathcal{S}}$, and work directly with the twistor space $\mathcal{Z}_{\mathcal{M}}$ of the QK manifold $\mathcal{M}$, as

\footnotetext{
2 See [7-9] for other discussions of superconformal invariance in projective superspace and [10,11] for an analysis in components.
} 
emphasized in particular by Salamon and Lebrun [12,14-16]. While $\mathcal{Z}_{\mathcal{S}}$ carries a complex $\mathcal{O}(2)$-valued symplectic structure and a HK metric degenerate along the $\mathbb{C} P^{1}$ fiber, $\mathcal{Z}_{\mathcal{M}}$ carries a complex $\mathcal{O}(2)$-valued contact structure and a non-degenerate Kähler-Einstein metric ${ }^{3}$ [12]. Conversely, Fano contact manifolds with a Kähler-Einstein metric are twistor spaces of QK manifolds $[13,14]^{4}$. Similarly to $\mathcal{Z}_{\mathcal{S}}$, the complex contact structure $\mathcal{X}$ on $\mathcal{Z}_{\mathcal{M}}$ admits local Darboux coordinates $\xi_{[i]}^{\Lambda}, \tilde{\xi}_{\Lambda}^{[i]}, \alpha^{[i]}(\Lambda=0, \ldots, d-1)$ such that locally, the contact one-form takes the canonical form $\mathcal{X}^{[i]}=\mathrm{d} \alpha^{[i]}+\xi_{[i]}^{\Lambda} \mathrm{d} \tilde{\xi}_{\Lambda}^{[i]}$. These Darboux coordinates on $\mathcal{Z}_{\mathcal{M}}$ are essentially the projectivization ${ }^{5}$ of the Darboux coordinates $v_{[i]}^{I}, \mu_{I}^{[i]}$ on $\mathcal{Z}_{\mathcal{S}}$. More precisely, we show that the projectivized complex Darboux coordinates depend on the coordinates $\left(\zeta, \pi^{1}, \pi^{2}\right)$ on the $\mathbb{C} P^{1} \times\left(\mathbb{C}^{2} / \mathbb{Z}_{2}\right)$ fiber over a given point on $\mathcal{M}$ only through the ratio $\mathbf{z}$ defined in (2.57) below. Together with the projectivization, this provides the desired reduction from $\mathcal{Z}_{\mathcal{S}}$ to $\mathcal{Z}_{\mathcal{M}}$. The homogeneous generating functions $S^{[i j]}\left(v_{[i]}^{I}, \mu_{I}^{[i]}, \zeta\right)$ of complex symplectomorphisms on $\mathcal{Z}_{\mathcal{S}}$ yield generating functions $\hat{S}^{[i j]}\left(\xi_{[i]}^{\Lambda}, \tilde{\xi}_{\Lambda}^{[i]}, \alpha^{[i]}\right)$ of complex contact transformations on $\mathcal{Z}$. Their deformations can be encoded in functions $\hat{H}_{(1)}^{[i j]}$ of the same variables, subject to consistency relations, reality conditions, and gauge equivalence. This recovers Lebrun's assertion that the QK deformations of $\mathcal{M}$ are classified by the Čech cohomology group $H^{1}\left(\mathcal{Z}_{\mathcal{M}}, \mathcal{O}(2)\right)$ [14]. The deformed QK metric on $\mathcal{M}$ can then be extracted in a systematic way from the knowledge of the "contact twistor lines" (referred to as the "twistor map" in [17]), i.e. the complex coordinates $\xi_{[i]}^{\Lambda}, \tilde{\xi}_{\Lambda}^{[i]}, \alpha^{[i]}$ on $\mathcal{Z}_{\mathcal{M}}$ expressed as functions of the coordinates $\mathbf{z}$ on $\mathbb{C} P^{1}$ and $x^{\mu}$ on the base $\mathcal{M}$.

We end this introduction with an important remark. In string theory or supergravity, only QK manifolds with negative scalar curvature appear as a consequence of supersymmetry [18]. Such QK spaces are generically non-compact. The linear deformation theory set up in this paper is local and applies to both compact and non-compact manifolds. Possible obstructions to extend and integrate infinitesimal deformations into finite global deformations, however, depend strongly on the (non-)compactness. For instance, it is known that complete QK manifolds with positive scalar curvature admit no deformations, see e.g. $[15,16]$. In contrast, the hypermultiplet moduli spaces arising from string theory compactifications are in general deformed by quantum corrections, as explained e.g. in the introduction of [1] and to be discussed further in [19].

This paper is organized as follows. • In Sect. 2, we review general aspects of QK manifolds, their twistor spaces, HKC and twistor spaces thereof, and study the consequences of superconformal invariance on the symplectomorphisms used in the patchwork construction of the complex symplectic structure. In particular, in Sect. 2.5, we explain in detail how the homogeneous complex symplectic structure on $\mathcal{Z}_{\mathcal{S}}$ reduces to a complex contact structure on $\mathcal{Z}_{\mathcal{M}}$, thus allowing to by-pass the Swann bundle and its twistor space. $\bullet$ In Sect. 3 we specify the case when the $4 d$-dimensional QK space has $d+1$ commuting isometries, i.e. when its Swann bundle is obtainable by the Legendre transform construction. We find the corresponding restriction on the symplectomorphisms, perform the superconformal quotient and obtain the contact twistor lines. • In Sect. 4, we illustrate these methods on the example of the hypermultiplet moduli space in type

\footnotetext{
3 Moreover, in contrast to $\mathcal{Z}_{\mathcal{S}}$, the projection from $\mathcal{Z}_{\mathcal{M}}$ to $\mathbb{C} P^{1}$ is not holomorphic.

4 In fact, our local analysis seems to support Lebrun's conjecture [14] that every Fano contact manifold is a twistor space.

5 The equivalence between contact structures and homogeneous symplectic structures is a standard trick in contact geometry, see e.g. [14] and references therein.
} 
II string theory, both at tree and one-loop level, and in the process strengthen the case for the absence of perturbative corrections beyond one-loop. • Section 5 studies deformations of QK manifolds with $d+1$ commuting isometries. We determine the allowed linear perturbations which preserve superconformal invariance, and find the deformed twistor lines and contact twistor lines. These results will be applied to the hypermultiplet moduli space of type II string theories in [19]. • In Appendix A, we spell out the $S U(2)$ action on the various multiplets at the infinitesimal level. In Appendix B, we briefly discuss an alternative description of the hypermultiplet moduli space using a different choice of contour, and show that it is related to the one in Sect. 4 by a local symplectomorphism. In Appendix C we generalize the superconformal quotient of Sect. 3.2 to the perturbed case, and provide an independent check on the results of Sect. 5.2.

\section{Quaternionic-Kähler Geometry and Twistors}

In this section, we review the relation between quaternionic-Kähler $(\mathrm{QK})$ manifolds and hyperkähler cones (HKC). This relation is one-to-one up to coverings (Theorem (5.9) in [2]), and can be established "bottom up", by constructing the Swann bundle $\mathcal{S}$ over the QK manifold $\mathcal{M}$, or "top down", by performing the superconformal quotient of $\mathcal{S}$. These two constructions are summarized in Sects. 2.1 and 2.2 following $[4,17]$. In Sect. 2.3, we recall the patchwork construction of the twistor space $\mathcal{Z}_{\mathcal{S}}$ of the HK space $\mathcal{S}$ developed in our previous work [1]. In Sect. 2.4 we derive the restrictions on the transition functions imposed by superconformal invariance. In Sect. 2.5, we study the reduction of the homogeneous complex symplectic structure on $\mathcal{Z}_{\mathcal{S}}$ to a complex contact structure on $\mathcal{Z}_{\mathcal{M}}$. The reader will find it helpful to refer to Fig. 1 for the various coordinate systems involved in these constructions.

2.1. Bottom-up: from $Q K$ to $H K C$. A quaternionic-Kähler manifold $\mathcal{M}$ is a $4 d$-dimensional manifold with Riemannian metric $g_{\mathcal{M}}$ and Levi-Civita connection $\nabla$ whose holonomy group is contained in $U S p(d) \times S U(2)$ [12]. $\mathcal{M}$ admits a triplet of almost complex Hermitian structures $\vec{J}$ (defined up to $S U(2)$ rotations) satisfying the algebra of the unit imaginary quaternions. The quaternionic two-forms $\vec{\omega}_{\mathcal{M}}(X, Y)=g_{\mathcal{M}}(\vec{J} X, Y)$ are covariantly closed with respect to the $S U(2)$ part $\vec{p}$ of the Levi-Civita connection, and are proportional with a fixed coefficient $v$ to the curvature of $\vec{p}$,

$$
\mathrm{d} \vec{\omega}_{\mathcal{M}}+\vec{p} \times \vec{\omega}_{\mathcal{M}}=0, \quad \mathrm{~d} \vec{p}+\frac{1}{2} \vec{p} \times \vec{p}=\frac{1}{2} v \vec{\omega}_{\mathcal{M}}
$$

where we use the notation $(\vec{a} \times \vec{b})_{i}=\epsilon_{i j k} a_{j} \wedge b_{k}$. As a consequence, the metric on $\mathcal{M}$ is Einstein, with constant Ricci scalar curvature $R=4 d(d+2) v$. HK manifolds are degenerate limits of QK manifolds, where $v=0$. We are mainly concerned in this work with the case of negative curvature, $v<0$.

The Swann bundle $\mathcal{S}$ associated to $\mathcal{M}$ is the total space of a $\mathbb{C}^{2}$ bundle (more precisely $\mathbb{C}^{2} / \mathbb{Z}_{2}$ with the zero section deleted) over $\mathcal{M}$. It is a hyperkähler manifold of dimension $4(d+1)$ with an $S U(2)$ isometric action which rotates the complex structures into each other, and a homothetic Killing vector. The homothetic Killing vector ensures that the hyperkähler manifold is actually a cone, and the $S U(2)$ isometries guarantee that this is a cone over a three-Sasakian space with $S^{3}$ fibres over the quaternionic base $\mathcal{M}$ [20]. In physics terminology, these properties follow from $\mathcal{N}=2$ superconformal 
invariance of the associated sigma model [4]. We denote by $\pi^{A^{\prime}}$ the complex coordinates on the $\mathbb{C}^{2} / \mathbb{Z}^{2}$ fiber, $\bar{\pi}_{A^{\prime}} \equiv\left(\pi^{A^{\prime}}\right)^{*}$ their complex conjugate, and use the antisymmetric tensor $\epsilon_{A^{\prime} B^{\prime}}$ to raise and lower the indices. ${ }^{6}$ The HK metric on $\mathcal{S}$ is given by

$$
\mathrm{d} s_{\mathcal{S}}^{2}=\left|D \pi^{A^{\prime}}\right|^{2}+\frac{v}{4} r^{2} \mathrm{~d} s_{\mathcal{M}}^{2}
$$

where $\mathrm{d} s_{\mathcal{M}}^{2}$ is the QK metric on $\mathcal{M}, r^{2} \equiv\left|\pi^{1}\right|^{2}+\left|\pi^{2}\right|^{2}=\pi^{A^{\prime}} \bar{\pi}_{A^{\prime}}$ is the squared norm on the fiber, and

$$
D \pi^{A^{\prime}} \equiv \mathrm{d} \pi^{A^{\prime}}+p^{A^{\prime}}{ }_{B^{\prime}} \pi^{B^{\prime}},
$$

is the covariant differential of $\pi^{A^{\prime}}$. The isometric $S U(2)$ action on $\mathcal{S}$ is given by the infinitesimal transformations

$$
\delta \pi^{A^{\prime}}=\frac{\mathrm{i}}{2} \epsilon_{3} \pi^{A^{\prime}}+\epsilon_{+} \bar{\pi}_{A^{\prime}}, \quad \delta \bar{\pi}_{A^{\prime}}=-\frac{\mathrm{i}}{2} \epsilon_{3} \bar{\pi}_{A^{\prime}}+\epsilon_{-} \pi_{A^{\prime}} .
$$

In particular, the norm $r^{2}$ is $S U(2)$ invariant. The homothetic Killing vector $r \partial_{r}=$ $\pi^{A^{\prime}} \partial_{\pi^{A^{\prime}}}+\bar{\pi}_{A^{\prime}} \partial_{\bar{\pi}_{A^{\prime}}}$ corresponds to dilations of the fiber. With respect to the complex structure $J^{3}$, where $D \pi^{A^{\prime}}$ are $(1,0)$ forms, the Kähler form is

$$
\omega_{\mathcal{S}}^{3}=\mathrm{i}\left(\mathrm{D} \pi^{A^{\prime}} \wedge \mathrm{D} \bar{\pi}_{A^{\prime}}+\frac{v}{2} \pi_{A^{\prime}} \bar{\pi}_{B^{\prime}} \omega_{\mathcal{M}}^{A^{\prime} B^{\prime}}\right)
$$

while the holomorphic symplectic form $\omega_{\mathcal{S}}^{+}=-\frac{1}{2}\left(\omega_{\mathcal{S}}^{1}-\mathrm{i} \omega_{\mathcal{S}}^{2}\right)$ is given by

$$
\omega_{\mathcal{S}}^{+}=\mathrm{D} \pi^{A^{\prime}} \wedge \mathrm{D} \pi_{A^{\prime}}+\frac{v}{2} \pi_{A^{\prime}} \pi_{B^{\prime}} \omega_{\mathcal{M}}^{A^{\prime} B^{\prime}}=\mathrm{d}\left(\pi_{A^{\prime}} \mathrm{D} \pi^{A^{\prime}}\right) .
$$

This construction directly defines the HKC, or Swann bundle $\mathcal{S}$, given a QK manifold $\mathcal{M}$, see [17] for more details.

For many purposes, it is useful to decompose the construction above in two steps, by first introducing the twistor space $\mathcal{Z}_{\mathcal{M}}[12]$, a $\mathbb{C} P^{1}$ bundle over $\mathcal{M}$, and then obtaining the $S$ wann bundle $\mathcal{S}$ as a $\mathbb{C}^{\times}$bundle over $\mathcal{Z}_{\mathcal{M}}$. The twistor space $\mathcal{Z}_{\mathcal{M}}$ over $\mathcal{M}$ should not be confused with the twistor space $\mathcal{Z}_{\mathcal{S}}$ of $\mathcal{S}$ itself, to be introduced in Sect. 2.3 below. $\mathcal{Z}_{\mathcal{M}}$ is a complex manifold with a canonical Kähler-Einstein metric and a complex contact structure ${ }^{7}$. Introducing a complex coordinate $z$ on $\mathbb{C} P^{1}$, the line element is given by

$$
\mathrm{d} s_{\mathcal{Z}_{\mathcal{M}}}^{2}=\frac{|\mathrm{d} z+\mathcal{P}|^{2}}{(1+z \bar{z})^{2}}+\frac{v}{4} \mathrm{~d} s_{\mathcal{M}}^{2},
$$

while the Kähler form on $\mathcal{Z}_{\mathcal{M}}$ is given by

$$
\omega_{\mathcal{Z}_{\mathcal{M}}}=\mathrm{i}\left(\frac{(\mathrm{d} z+\mathcal{P}) \wedge(\mathrm{d} \bar{z}+\overline{\mathcal{P}})}{(1+z \bar{z})^{2}}+\frac{v}{2} \frac{(1-z \bar{z}) \omega_{\mathcal{M}}^{3}-2 \mathrm{i} z \omega_{\mathcal{M}}^{+}+2 \mathrm{i} \bar{z} \omega_{\mathcal{M}}^{-}}{1+z \bar{z}}\right),
$$

\footnotetext{
${ }^{6}$ We use conventions in which $\epsilon_{12}=1=-\epsilon_{21}$ and $\bar{\pi}_{A^{\prime}}=\bar{\pi}^{B^{\prime}} \epsilon_{B^{\prime} A^{\prime}}$.

7 Recall that a complex contact form on a complex manifold of complex dimension $2 d+1$ is a holomorphic one-form $\hat{\mathcal{X}}$, defined globally, such that $\hat{\mathcal{X}} \wedge(\mathrm{d} \hat{\mathcal{X}})^{d}$ is a nowhere vanishing holomorphic top form. A contact structure corresponds to the case where $\hat{\mathcal{X}}$ is a local one-form defined up to multiplication by a nowhere vanishing smooth function.
} 
where $\omega_{\mathcal{M}}^{ \pm}=-\frac{1}{2}\left(\omega_{\mathcal{M}}^{1} \mp \mathrm{i} \omega_{\mathcal{M}}^{2}\right), \omega_{\mathcal{M}}^{+}=\left(\omega_{\mathcal{M}}^{-}\right)^{*}$. In these expressions, $\mathcal{P}$ stands for the "projectivized connection", defined from the $S U(2)$ connection $p^{A^{\prime}} B^{\prime}$ as

$$
\mathcal{P}=p_{+}-\mathrm{i} p_{3} z+p_{-} z^{2}
$$

where $p_{+} \equiv p_{2}{ }_{2}, p_{3} \equiv \mathrm{i}\left(p^{1}{ }_{1}-p^{2}{ }_{2}\right), p_{-} \equiv-p^{2}{ }_{1}$, with $p_{3}$ real and $\left(p_{-}\right)^{*}=p_{+}$.

The complex contact structure on $\mathcal{Z}_{\mathcal{M}}$ is induced from the Liouville form $\mathcal{X}$ on $\mathcal{S}$,

$$
\mathcal{X} \equiv \pi_{A^{\prime}} \mathrm{D} \pi^{A^{\prime}}=\frac{1}{2}\left(\pi^{2}\right)^{2}(\mathrm{~d} z+\mathcal{P})
$$

and, as apparent from the overall factor of $\left(\pi^{2}\right)^{2}$, is a section ${ }^{8}$ of the $\mathcal{O}(2)$ line bundle on $\mathbb{C} P^{1}$. From the complex contact structure one may easily extract the $S U(2)$ connection $\vec{p}$, and therefore the triplet of quaternionic two-forms $\vec{\omega}_{\mathcal{M}}$ via Eq. (2.1). Thus, the knowledge of the complex structure and contact structure on $\mathcal{Z}_{\mathcal{M}}$ is sufficient to reconstruct the quaternionic-Kähler metric.

To construct the Swann bundle $\mathcal{S}$ we introduce two more real coordinates $r$ and $\psi$ parametrizing the fiber of a $\mathbb{C}^{\times}$bundle over $\mathcal{Z}_{\mathcal{M}}$, with metric ${ }^{9}$

$$
\mathrm{d} s_{\mathcal{S}}^{2}=\mathrm{d} r^{2}+r^{2}\left[(D \psi)^{2}+\mathrm{d} s_{\mathcal{Z}_{\mathcal{M}}}^{2}\right]
$$

where

$$
D \psi=\mathrm{d} \psi+\frac{\mathrm{i}}{2(1+z \bar{z})}\left[(z \mathrm{~d} \bar{z}-\bar{z} \mathrm{~d} z)-\mathrm{i}(1-z \bar{z}) p_{3}+2 z p_{-}-2 \bar{z} p_{+}\right]
$$

The metrics (2.2) and (2.11) are identical, provided the coordinates $r, \psi, z, \bar{z}$ are related to $\pi^{A^{\prime}}, \bar{\pi}_{A^{\prime}}$ via

$$
r^{2}=\left|\pi^{1}\right|^{2}+\left|\pi^{2}\right|^{2}, \quad e^{i \psi}=\sqrt{\pi^{2} / \bar{\pi}_{2}}, \quad z=\frac{\pi^{1}}{\pi^{2}}, \quad \bar{z}=\frac{\bar{\pi}_{1}}{\bar{\pi}_{2}},
$$

or conversely

$$
\left(\begin{array}{l}
\pi^{1} \\
\pi^{2}
\end{array}\right)=\frac{r e^{\mathrm{i} \psi}}{\sqrt{1+z \bar{z}}}\left(\begin{array}{l}
z \\
1
\end{array}\right)
$$

For more details, we again refer the reader to [17].

\footnotetext{
${ }^{8}$ More precisely, $\mathcal{X}$ is defined on $\mathcal{S}$. In (2.23) we define a contact one-form $\hat{\mathcal{X}}$ proportional to $\mathcal{X}$, which does live on $\mathcal{Z}_{\mathcal{M}}$.

9 We follow the conventions of [17], but with a slightly different notation. E.g. the coordinate $\psi$ here is denoted by $\phi$ in [17]. Also, in [17], the $S U(2)$ index in $\pi^{A^{\prime}}$ was not lowered after complex conjugation.
} 
2.2. Top down: from $H K C$ to $Q K$. The characterizing property of an $\mathrm{HKC}$ is that there exists a function $\chi$ on $\mathcal{S}$, known as the hyperkähler potential, such that the metric, in local (real) coordinates $\phi^{M}, M=1, \ldots, 4(d+1)$, satisfies [2,4]

$$
g_{M N}=D_{M} \partial_{N} \chi(\phi) .
$$

For any Hermitian complex structure, in adapted complex coordinates $z^{m}, m=1, \ldots$, $2(d+1),(2.15)$ implies that

$$
g_{m \bar{n}}=\partial_{m} \partial_{\bar{n}} \chi(z, \bar{z}), \quad D_{m} \partial_{n} \chi(z, \bar{z})=0 .
$$

In particular, $\chi$ provides a Kähler potential in any complex structure. The dilation and $S U(2)$ symmetries are generated by the vector fields

$$
\chi^{M}=g^{M N} \chi_{N}, \quad \vec{k}^{M}=\vec{J}_{N}^{M} \chi^{N},
$$

where $\chi_{M} \equiv \partial_{M} \chi, g^{M N}$ is the inverse HKC metric, and $\vec{J}$ is a triplet of complex structures. The $S U(2)$ Killing vector fields are not tri-holomorphic but rotate the complex structures into each other. It follows from (2.15) that the four vector fields $\chi^{M}$ and $\vec{k}^{N}$ satisfy

$$
D_{M} \chi^{N}=\delta_{M}^{N}, \quad D_{M} \vec{k}^{N}=\vec{J}_{M}^{N} .
$$

In particular, $\chi^{m} \partial_{z^{m}}$ is holomorphic. One can also express the hyperkähler potential in terms of the metric and the homothetic Killing vector fields,

$$
\chi=\frac{1}{2} \chi^{M} g_{M N} \chi^{N}=\frac{1}{2} \chi^{M} \partial_{M} \chi
$$

consistent with (2.15). It is easy to check that this form of the hyperkähler potential is $S U$ (2) invariant. In the coordinates that appear in the construction of the Swann bundle, the homothetic Killing vector is generated by the vector field $\chi^{M} \partial_{M}=r \partial_{r}$, and so (2.19) yields

$$
\chi=r^{2}=\pi^{A^{\prime}} \bar{\pi}_{A^{\prime}} .
$$

One can descend from the HKC $\mathcal{S}$ to the twistor space $\mathcal{Z}_{\mathcal{M}}$ by performing a $U(1)$ Kähler quotient. For any choice of complex structure $\vec{n} \cdot \vec{J}$ with $\vec{n}$ a unit vector, $\vec{n} \cdot \vec{k}$ is a holomorphic Killing vector. The Kähler quotient of $\mathcal{Z}_{\mathcal{M}}$ with respect to $\vec{n} \cdot \vec{k}$ provides a Kähler manifold of real dimension $4 d+2$, independent of the choice of $\vec{n}$, which is just the twistor space $\mathcal{Z}_{\mathcal{M}}$. By Frobenius' theorem, one may choose a set of independent complex coordinates $\lambda, u^{i}, i=1, \ldots, 2 d+1$ adapted to the action of the holomorphic vector field $\chi^{M}$,

$$
\chi^{m}(z) \partial_{m}=\left.\partial_{\lambda}\right|_{u^{i}}
$$

The Kähler potential on $\mathcal{Z}_{\mathcal{M}}$ is then determined from the hyperkähler potential $\chi$ by means of

$$
\chi(\lambda, \bar{\lambda}, u, \bar{u})=e^{\lambda+\bar{\lambda}+K_{\mathcal{Z}_{\mathcal{M}}}}(u, \bar{u}) .
$$

Defining the $\mathcal{O}(2)$-twisted holomorphic contact form on $\mathcal{Z}_{\mathcal{M}}$,

$$
\hat{\mathcal{X}} \equiv e^{-2 \lambda} \mathcal{X}=e^{\bar{\lambda}-\lambda+2 \mathrm{i} \psi+K_{\mathcal{Z}}} \frac{\mathrm{d} z+\mathcal{P}}{2(1+z \bar{z})},
$$


one may rewrite the metric on the fiber as the modulus square of the contact form [3],

$$
\frac{|\mathrm{d} z+\mathcal{P}|^{2}}{(1+z \bar{z})^{2}}=4 e^{-2 K_{\mathcal{Z}}}|\hat{\mathcal{X}}|^{2} .
$$

Note that $\psi$ is not an independent coordinate, but rather will be determined in terms of $\lambda, z, x^{\mu}$ in Eq. (2.79) below, in such a way that $\bar{\lambda}-\lambda+2 \mathrm{i} \psi$ is a function on $\mathcal{Z}_{\mathcal{M}}$ only.

The QK metric on $\mathcal{M}$ can be computed from the holomorphic contact form $\mathcal{X}$ as indicated below (2.10), or by decomposing the metric on the twistor space as in (2.7), see [4,5] for more details. To express the metric on $\mathcal{M}$ in closed form, one needs to express the complex coordinates $z^{m}$ on $\mathcal{S}$ (or $u^{i}$ on $\mathcal{Z}_{\mathcal{M}}$ ) in terms of $4 d$ independent real coordinates, corresponding to $\mathbb{R}^{+} \times S U(2)$ invariant combinations of $\phi^{M}$, and coordinates on the $\mathbb{C}^{2}$ fiber $z, \bar{z}, \lambda, \bar{\lambda}$. As we shall see shortly, this problem is a $\mathrm{QK}$ analog of the problem of "parametrizing the twistor lines" in HK geometry.

2.3. Patchwork construction of twistor spaces of HK manifolds - a summary. As explained e.g. in [1,21-25], HK geometry is equivalent to complex symplectic geometry on the twistor space, compatible with the real structure. This, of course, also applies to the HKC metric on the Swann bundle $\mathcal{S}$, with suitable restrictions on the complex symplectic structure to ensure the HK cone property. In this subsection, we briefly review the twistorial description of general HK manifolds $\mathcal{S}$ following [1], before studying the implications of superconformal invariance in Sect. 2.4.

In contrast to the quaternionic-Kähler case described in Sect. 2.1, the twistor space $\mathcal{Z}_{\mathcal{S}}$ over a $4 d+4$-dimensional $\mathrm{HK}$ manifold ${ }^{10} \mathcal{S}$ is a trivial product $\mathcal{Z}_{\mathcal{S}}=\mathcal{S} \times \mathbb{C} P^{1}$. Its structure was developed from a physics viewpoint in [22,24], and its relation to projective superspace was recently further analysed in [25].

We denote by $\zeta$ a complex coordinate on the projective line $\mathbb{C} P^{1}$ around the north pole $\zeta=0 . \mathcal{Z}_{\mathcal{S}}$ carries an integrable complex structure given by

$$
J(\zeta, \bar{\zeta})=\frac{1-\zeta \bar{\zeta}}{1+\zeta \bar{\zeta}} J^{3}+\frac{\zeta+\bar{\zeta}}{1+\zeta \bar{\zeta}} J^{2}+i \frac{\zeta-\bar{\zeta}}{1+\zeta \bar{\zeta}} J^{1}
$$

on the base $\mathcal{S}$ (where $J_{i}$ are the three complex structures on $\mathcal{S}$ ) and the standard complex structure on $\mathbb{C} P^{1}$. Moreover, in this complex structure, $\mathcal{Z}_{\mathcal{S}}$ carries a holomorphic two-form (more accurately, a section of $\Lambda^{2} T_{F}^{*}(2)$, see [22]) and a Kähler form given locally by

$$
\Omega(\zeta)=\omega_{\mathcal{S}}^{+}-\mathrm{i} \zeta \omega_{\mathcal{S}}^{3}+\zeta^{2} \omega_{\mathcal{S}}^{-}
$$

and

$$
\omega(\zeta, \bar{\zeta})=\frac{1}{1+\zeta \bar{\zeta}}\left[(1-\zeta \bar{\zeta}) \omega_{\mathcal{S}}^{3}-2 \mathrm{i} \zeta \omega_{\mathcal{S}}^{+}+2 \mathrm{i} \bar{\zeta} \omega_{\mathcal{S}}^{-}\right]
$$

where $\omega_{\mathcal{S}}^{ \pm}=-\frac{1}{2}\left(\omega_{\mathcal{S}}^{1} \mp \mathrm{i} \omega_{\mathcal{S}}^{2}\right)$. Note that, in contrast to the quaternionic-Kähler case, both of these forms are degenerate along the $\mathbb{C} P^{1}$ fiber direction $\mathrm{d} \zeta$. The Kähler form $\omega$ coincides with $\omega_{\mathcal{S}}^{3}$ at the north pole $\zeta=0$, and with $-\omega_{\mathcal{S}}^{3}$ at the south pole $\zeta=\infty$.

\footnotetext{
$\mathcal{M}$.

${ }^{10}$ For obvious reasons, we deviate from the notations of [1] which considered $4 d$ dimensional HK manifolds
} 
The holomorphic two-form $\Omega$ however, while coinciding with $\omega_{\mathcal{S}}^{+}$at the north pole, diverges with a second order pole at $\zeta=\infty$. As explained in [1], it is useful to introduce a set of patches $\hat{\mathcal{U}}_{i}, i=1, \ldots, N$ on $\mathcal{Z}_{\mathcal{S}}$, which project to open disks ${ }^{11} \mathcal{U}_{i}$ on $\mathbb{C} P^{1}$, and a local section $\Omega^{[i]}$ which is regular on each patch. In order for the holomorphic section $\Omega$ to be well defined, one must require that, on the overlap $\hat{\mathcal{U}}_{i} \cap \hat{\mathcal{U}}_{j}$,

$$
\Omega^{[i]}=f_{i j}^{2}(\zeta) \Omega^{[j]} \quad \bmod \mathrm{d} \zeta .
$$

The factor $f_{i j}(\zeta)$ corresponds to the transition function of the $\mathcal{O}(1)$ bundle on $\mathbb{C} P^{1}$, and was discussed in detail in [1]. In particular, we recall that

$$
f_{i j} f_{j k}=f_{i k}, \quad f_{i i}=1, \quad \overline{\tau\left(f_{i j}^{2}\right)}=f_{\bar{l} \bar{j}}^{2},
$$

where $\tau$ is the antipodal map $[\tau(\nu)](\zeta) \equiv v(-1 / \bar{\zeta})$, and $\bar{l}$ labels the patch $\mathcal{U}_{\bar{l}}$ opposite to the patch $\mathcal{U}_{i}$ under the involution $\tau$. Defining $\Omega^{[0]}=\Omega$ and using $f_{0 \infty}=\zeta$ one finds that

$$
\Omega^{[\infty]} \equiv \zeta^{-2} \Omega^{[0]}=\omega_{\mathcal{S}}^{-}-\mathrm{i} \omega_{\mathcal{S}}^{3} \zeta^{-1}+\omega_{\mathcal{S}}^{+} \zeta^{-2}
$$

is regular at the south pole $\zeta=\infty$.

Now, we may choose the covering $\hat{\mathcal{U}}_{i}$ such that, on each patch, the holomorphic section $\Omega^{[i]}$ takes the Darboux form

$$
\Omega^{[i]}=\mathrm{d} \mu_{I}^{[i]} \wedge \mathrm{d} \nu_{[i]}^{I},
$$

where $\left(v_{[i]}^{I}, \mu_{I}^{[i]}, \zeta\right)$ is a local complex coordinate system on $\mathcal{Z}_{\mathcal{S}}$, regular throughout the patch $\hat{\mathcal{U}}_{i}$ (here $I$ runs over $d+1$ values, which we shall denote $b, 0, \ldots, d-1$ ). Equation (2.28) implies that on the overlap of two patches, $\left(v_{[i]}^{I}, \mu_{I}^{[i]}\right)$ and $\left(v_{[j]}^{I}, \mu_{I}^{[j]}\right)$ must be related by a complex $(\mathcal{O}(2)$-twisted) symplectomorphism. This is conveniently encoded by a generating function $S^{[i j]}$ of the initial "position" and final "momentum" coordinates, such that

$$
v_{[j]}^{I}=\partial_{\mu_{I}^{[j]}} S^{[i j]}\left(v_{[i]}, \mu^{[j]}, \zeta\right), \quad \mu_{I}^{[i]}=f_{i j}^{2} \partial_{v_{[i]}^{I}} S^{[i j]}\left(v_{[i]}, \mu^{[j]}, \zeta\right) .
$$

To check (2.28), one may use the identity

$$
\mathrm{d} S^{[i j]}=v_{[j]}^{I} \mathrm{~d} \mu_{I}^{[j]}+f_{i j}^{-2} \mu_{I}^{[i]} \mathrm{d} \nu_{[i]}^{I} \quad \bmod \mathrm{d} \zeta .
$$

The transition functions $S^{[i j]}$ are restricted by consistency conditions which ensure that the symplectomorphisms compose properly (see [1] for more details). As a result, the holomorphic symplectic structure on the twistor space $\mathcal{Z}_{\mathcal{S}}$ is entirely specified by $N-1$ freely chosen functions $S^{[0 i]}\left(\nu_{[0]}, \mu^{[i]}, \zeta\right)$. In order to ensure the reality of the resulting metric, it is also necessary to require that the sections $v_{[i]}^{I}, \mu_{I}^{[i]}$ transform under the real structure as

$$
\overline{\tau\left(v_{[i]}^{I}\right)}=-v_{[\bar{i}]}^{I}, \quad \overline{\tau\left(\mu_{I}^{[i]}\right)}=-\mu_{I}^{[\bar{l}]} .
$$

11 In principle, one should introduce a local coordinate $\zeta^{[i]}$ on each connected disk; to avoid cluttering we shall use a single coordinate $\zeta$ to parametrize all patches at once, with each connected disk $\mathcal{U}_{i}$ being centered at $\zeta=\zeta_{i}$. 
The condition (2.34) requires that the functions $S^{[i \bar{i}]}$ are related by complex conjugation to their Legendre transform [1].

For a suitably generic choice of such transition functions, it is a general property of HK manifolds that the space of solutions of (2.32) has dimension $4 d+4$, i.e. all $v_{[i]}^{I}$, $\mu_{I}^{[i]}$ can be expressed as infinite Taylor series around $\zeta=\zeta_{i}$ whose coefficients are all functions of $4 d+4$ parameters. The moduli space of solutions is isomorphic to the $\mathrm{HK}$ base $\mathcal{S}$, and the map $\zeta \mapsto\left(v_{[i]}^{I}, \mu_{I}^{[i]}\right)$ defines the "twistor lines", i.e. realizes the $\mathbb{C} P^{1}$ fiber over any point in $\mathcal{S}$ as a rational curve in $\mathcal{Z}_{\mathcal{S}}$.

Having found the twistor lines, the geometry of $\mathcal{S}$ can be computed by Taylor expanding the holomorphic section $\Omega$ around any point $\zeta \in \mathbb{C} P^{1}$. When $\mathcal{S}$ is a HKC, as we discuss further in the next section, all points of $\mathbb{C} P^{1}$ are equivalent, and we can therefore expand around $\zeta=0$. Since $\Omega$ is a global section of $\mathcal{O}(2)$, the Taylor expansion stops at quadratic order,

$$
\Omega^{[0]}=\mathrm{d} w_{I} \wedge \mathrm{d} v^{I}-\mathrm{i} \omega_{\mathcal{S}}^{3} \zeta+\mathrm{d} \bar{w}_{I} \wedge \mathrm{d} \bar{v}^{I} \zeta^{2},
$$

where $v^{I}, w_{I}$ are the complex coordinates in the complex structure $J^{3}=J(0,0)$,

$$
v^{I}=v_{[0]}^{I}(\zeta=0), \quad w_{I}=\mu_{I}^{[0]}(\zeta=0) .
$$

Knowing the complex coordinates and the Kähler form $\omega_{\mathcal{S}}^{3}$, it is straightforward to obtain the metric and a Kähler potential. When $\mathcal{S}$ is a HKC, as we will discuss in the next section, it is always possible to choose the Kähler potential such that it is invariant under $S U(2)$, and therefore equal to the hyperkähler potential $\chi$.

2.4. Conditions for superconformal invariance. We now discuss the implications of superconformal invariance for the general construction of the twistor space $\mathcal{Z}_{\mathcal{S}}$ of a HK manifold $\mathcal{S}$. We recall from Sect. 2.2 that superconformal invariance requires the existence of a homothetic Killing vector and an $S U(2)$ group of Killing vectors that rotates the complex structures and commutes with the dilations.

As follows from the first equation in (2.18), the dilations rescale the hyperkähler cone metric and leave the complex structures invariant. We normalize the action of the dilations such that the metric has weight 2 ,

$$
g^{\prime}=\Lambda^{2} g, \quad \vec{J}^{\prime}=\vec{J} .
$$

This implies that all the two-forms $\vec{\omega}_{\mathcal{S}}$ on the Swann bundle $\mathcal{S}$ scale with weight two. The action of the dilations can be extended to the twistor space $\mathcal{Z}_{\mathcal{S}}$ by assigning a scaling weight zero to $\zeta$. In this way, the holomorphic two-form $\Omega$ from (2.26) transforms uniformly throughout the $\zeta$ plane,

$$
\Omega^{\prime[i]}=\Lambda^{2} \Omega^{[i]} .
$$

The local Darboux coordinates $v_{[i]}$ and $\mu^{[i]}$ must transform in such a way that $(2.38)$ is obeyed, so we postulate ${ }^{12}$

$$
v_{[i]}^{\prime I}=\Lambda^{2 n} v_{[i]}^{I}, \quad \mu_{I}^{\prime[i]}=\Lambda^{(2-2 n)} \mu_{I}^{[i]},
$$

\footnotetext{
12 One may also consider giving a different scaling weight $n_{I}$ for each conjugate pair $\left(v^{I}, \mu_{I}\right)$. The generalization of the following discussion is immediate.
} 
for some constant $n$. This is a symmetry of the gluing conditions (2.32) provided the generating functions are homogenous functions of degree one when $v$ and $\mu$ are scaled with degree $n$ and $1-n$ respectively,

$$
S^{[i j]}\left(\Lambda^{2 n} v_{[i]}, \Lambda^{(2-2 n)} \mu^{[j]}, \zeta\right)=\Lambda^{2} S^{[i j]}\left(v_{[i]}, \mu^{[j]}, \zeta\right)
$$

We now turn to the $S U(2)$ action. In order for the complex structure $J(\zeta, \bar{\zeta})$ given in $(2.25)$ to be invariant, one should compensate the rotation of $\vec{J}$ by a rotation on the $\mathbb{C} P^{1}$ fiber. Thus the fiber coordinate $\zeta$ must transform as

$$
\zeta^{\prime}=\frac{\alpha \zeta+\beta}{-\bar{\beta} \zeta+\bar{\alpha}}, \quad \alpha \bar{\alpha}+\beta \bar{\beta}=1
$$

Under this transformation, $\Omega$ should transform as a $\mathcal{O}(2)$ section,

$$
\Omega^{\prime[0]}(\zeta)=(-\bar{\beta} \zeta+\bar{\alpha})^{2} \Omega^{[0]}\left(\zeta^{\prime}\right)
$$

Here, we have written the action in the patch $\mathcal{U}_{0}$ around the north pole of $\mathbb{C} P^{1}$, parametrized by the local coordinate $\zeta=\zeta^{[0]}$. The action in the patch $\mathcal{U}_{i}$ can be obtained by replacing $\zeta \rightarrow \zeta^{[i]}, \Omega^{[0]} \rightarrow \Omega^{[i]}$. If we continue to use $\zeta$ as a coordinate in $\mathcal{U}_{i}$, then from (2.28) the transformation of $\Omega^{[i]}$ becomes

$$
\Omega^{\prime[i]}(\zeta)=\left[\frac{f_{i 0}(\zeta)}{f_{i 0}\left(\zeta^{\prime}\right)}\right]^{2}(-\bar{\beta} \zeta+\bar{\alpha})^{2} \Omega^{[i]}\left(\zeta^{\prime}\right)
$$

In order to ensure that $\Omega$ transforms as (2.43) in every patch, we postulate that the local Darboux coordinates $v_{[i]}^{I}, \mu_{I}^{[i]}$ transform locally as $\mathcal{O}(2 n)$ and $\mathcal{O}(2-2 n)$ sections

$$
\begin{aligned}
& v_{[i]}^{\prime I}(\zeta)=\left[\frac{f_{i 0}(\zeta)}{f_{i 0}\left(\zeta^{\prime}\right)}(-\bar{\beta} \zeta+\bar{\alpha})\right]^{2 n} v_{[i]}^{I}\left(\zeta^{\prime}\right), \\
& \mu_{I}^{\prime[i]}(\zeta)=\left[\frac{f_{i 0}(\zeta)}{f_{i 0}\left(\zeta^{\prime}\right)}(-\bar{\beta} \zeta+\bar{\alpha})\right]^{2-2 n} \mu_{I}^{[i]}\left(\zeta^{\prime}\right)
\end{aligned}
$$

This is a symmetry of the gluing equations (2.32) provided

$$
S^{[i j]}\left(v_{[i]}^{\prime}(\zeta), \mu^{\prime}[j](\zeta), \zeta\right)=\left[\frac{f_{j 0}(\zeta)}{f_{j 0}\left(\zeta^{\prime}\right)}\right]^{2}(-\bar{\beta} \zeta+\bar{\alpha})^{2} S^{[i j]}\left(v_{[i]}\left(\zeta^{\prime}\right), \mu^{[j]}\left(\zeta^{\prime}\right), \zeta^{\prime}\right)
$$

Using the homogeneity property (2.40), this translates into

$$
S^{[i j]}\left[\frac{f_{i j}^{2 n}(\zeta)}{f_{i j}^{2 n}\left(\zeta^{\prime}\right)} v_{[i]}\left(\zeta^{\prime}\right), \mu^{[j]}\left(\zeta^{\prime}\right), \zeta\right]=S^{[i j]}\left[v_{[i]}\left(\zeta^{\prime}\right), \mu^{[j]}\left(\zeta^{\prime}\right), \zeta^{\prime}\right] .
$$

This equation fixes the $\zeta$ dependence to be of the form

$$
S^{[i j]}\left(v_{[i]}, \mu^{[j]}, \zeta\right)=\hat{S}^{[i j]}\left(f_{i j}^{-2 n} v_{[i]}, \mu^{[j]}\right) .
$$


In particular, note that the special case where $v_{[i]}^{I}$ and $\mu_{I}^{[i]}$ are global sections of $\mathcal{O}(2 n)$ and $\mathcal{O}(2-2 n)$,

$$
S^{[i j]}\left(v_{[i]}, \mu^{[j]}, \zeta\right)=f_{i j}^{-2 n} v_{[i]}^{I} \mu_{I}^{[j]}
$$

solves the conditions of superconformal invariance. In addition, as in [1], one must impose the reality conditions

$$
\overline{\tau\left(S^{[i j]}\left(\nu_{[i]}, \mu^{[j]}, \zeta^{[i]}\right)\right)}=S^{[\bar{i} \bar{j}]}\left(\nu_{[\bar{i}]}, \mu^{[\bar{j}]}, \zeta^{[\bar{i}]}\right) .
$$

Thus, we conclude that superconformal invariance is guaranteed provided the generating functions $S^{[i j]}\left(v_{[i]}, \mu^{[j]}, \zeta\right)$ are functions of $f_{i j}^{-2 n} v_{[i]}$ and $\mu^{[j]}$, without explicit dependence on $\zeta$, homogeneous of degree 1 when their first and second arguments are scaled with weight $n$ and $1-n$, respectively, and satisfying the reality condition (2.49).

Anomalous $\mathcal{O}(0)$ multiplets. In fact, the above conditions are sufficient but not strictly speaking necessary. Indeed, we have assumed that the Darboux coordinates are adapted to the superconformal action, in the sense that dilations and $S U(2)$ act canonically as in (2.39) and (2.44), respectively. Clearly, a local gauge transformation depending on $\zeta$ only would not affect the existence of an isometric $S U(2)$ action, but would just make it look more complicated. More importantly, when $n=1$ (or equivalently $n=0$, after exchanging $\mu_{I}$ with $v^{I}$ ), it is possible that $\mu_{I}$ transforms anomalously under dilations, namely

$$
\mu_{I}^{\prime[i]}=\mu_{I}^{[i]}-c_{I}^{[i]} \log \Lambda^{2}
$$

for some constants $c_{I}^{[i]}$, which we shall refer to as "anomalous dimensions". This anomalous transformation may be generated from the standard transformation (2.39) with $n=0$ by a local symplectomorphism generated by

$$
T^{[i]}=\tilde{\mu}_{I}^{[i]} v_{[i]}^{I}-c_{I}^{[i]} v_{[i]}^{I} \log v_{[i]}^{b},
$$

where $v_{[i]}^{b}$ is any one of the $v_{[i]}^{I}$. This however need not be a regular gauge transformation in the patch $\mathcal{U}_{i}$, and so the geometry will in general depend non-trivially on $c_{I}^{[i]}$. After this local symplectomorphism, the generating functions $S^{[i j]}$ are now of the form ${ }^{13}$

$$
S^{[i j]}=f_{i j}^{-2}\left[\hat{S}^{[i j]}\left(v_{[i]}, \mu_{I}^{[j]}+c_{I}^{[j]} \log \left(f_{i j}^{-2} v_{[i]}^{b}\right)\right)-c_{I}^{[i]} v_{[i]}^{I} \log v_{[i]}^{b}\right],
$$

where $\hat{S}^{[i j]}$ is a homogeneous function of degree one in its first argument. In particular, $S^{[i j]}$ satisfy a "quasi-homogeneity condition"

$$
\begin{aligned}
S^{[i j]}\left(\Lambda^{2} v_{[i]}^{I}, \mu_{I}^{[j]}-c_{I}^{[j]} \log \Lambda^{2}, \zeta\right)= & \Lambda^{2}\left[S^{[i j]}\left(v_{[i]}^{I}, \mu_{I}^{[j]}, \zeta\right)\right. \\
& \left.-f_{i j}^{-2} c_{I}^{[i]} v_{[i]}^{I} \log \Lambda^{2}\right] .
\end{aligned}
$$

13 The $\hat{S}^{[i j]}$ appearing in this equation differs from the one in (2.47), the relation between the two being transcendental in general. 
Such generating functions are consistent with $S U(2)$ invariance and dilations provided $\mu_{I}^{[i]}$ transforms in the same way as $-c_{I}^{[i]} \log v_{[i]}^{b}$, namely as (2.50) under dilations and

$$
\mu_{I}^{\prime[i]}(\zeta)=\mu_{I}^{[i]}\left(\zeta^{\prime}\right)-2 c_{I}^{[i]} \log \left[\frac{f_{i 0}(\zeta)}{f_{i 0}\left(\zeta^{\prime}\right)}(-\bar{\beta} \zeta+\bar{\alpha})\right]
$$

under rotations. Anomalous transformations play an important role, e.g., in describing the one-loop correction to the hypermultiplet metric in Sect. 4.2.

Note that the constants $c_{I}^{[i]}$ are not arbitrary. Firstly, they must satisfy the reality conditions $\left(c_{I}^{[i]}\right)^{*}=-c_{I}^{[\bar{i}]}$. Besides, they are also subject to additional consistency constraints, which follow from the requirement that the open contours around the logarithmic branch cuts in $\zeta$ plane (as discussed in [1]) combine consistently into closed contours. This requires in particular that the anomalous dimensions associated with the patches containing the zeros of $v_{[i]}^{b}$ are real. In this paper we assume that $v_{[i]}^{b}$ has always two first order zeros $\zeta_{ \pm}$in the patches $\mathcal{U}_{ \pm}$related by the antipodal map, and therefore demand that $c_{I}^{[+]}=-c_{I}^{[-]}$are real constants. For a similar reason, we impose the same condition on $c_{I}^{[0]}=-c_{I}^{[\infty]}$ (see footnote 18$)$.

For later reference, we give the action of the symplectomorphism generated by (2.52),

$$
\begin{aligned}
& v_{[j]}^{I}=f_{i j}^{-2} \partial_{\mu_{I}^{[j]}} \hat{S}^{[i j]}, \\
& \mu_{I}^{[i]}=\partial_{v_{[i]}^{I}} \hat{S}^{[i j]}-c_{I}^{[i]} \log v_{[i]}^{b}+\delta_{I}^{b}\left(\frac{1}{v_{[i]}^{b}} c_{J}^{[j]} \partial_{\mu_{J}^{[j]}} \hat{S}^{[i j]}-\frac{v_{[i]}^{J}}{v_{[i]}^{b}} c_{J}^{[i]}\right) .
\end{aligned}
$$

From $\mathbb{C} P_{\zeta}^{1} \times \mathbb{C}_{\pi}^{2}$ to $\mathbb{C} P_{\mathbf{z}}^{1}$. We close this discussion of $S U(2)$ transformations with an important observation, which will be instrumental for understanding the relation between the twistor spaces $\mathcal{Z}_{\mathcal{S}}$ and $\mathcal{Z}_{\mathcal{M}}$. Notice that the isometric $S U$ (2) action on $\mathcal{S}$ corresponds to an $S U$ (2) action on the fiber coordinates $\pi^{A^{\prime}}, \bar{\pi}_{A^{\prime}}(2.4)$, at a fixed position on the QK base $\mathcal{M}$. Thus, any local $\mathcal{O}(2 n)$ section $v_{[i]}$, viewed as a function of $\left(\zeta, \pi^{A^{\prime}}, \bar{\pi}_{A^{\prime}}\right)$ and $x^{\mu}$, satisfies differential equations

$$
\begin{aligned}
\left(\partial_{\zeta}+\bar{\pi}_{1} \partial_{\pi^{2}}-\bar{\pi}_{2} \partial_{\pi^{1}}\right)\left(f_{i 0}^{-2 n} v_{[i]}\right) & =0, \\
\left(2 \zeta \partial_{\zeta}-2 n+\pi^{1} \partial_{\pi^{1}}+\pi^{2} \partial_{\pi^{2}}-\bar{\pi}_{1} \partial_{\bar{\pi}_{1}}-\bar{\pi}_{2} \partial_{\bar{\pi}_{2}}\right)\left(f_{i 0}^{-2 n} v_{[i]}\right) & =0 \\
\left(\zeta^{2} \partial_{\zeta}-2 n \zeta+\pi^{1} \partial_{\bar{\pi}_{2}}-\pi^{2} \partial_{\bar{\pi}_{1}}\right)\left(f_{i 0}^{-2 n} v_{[i]}\right) & =0 .
\end{aligned}
$$

It follows that there exists a function $\tilde{v}_{[i]}\left(\mathbf{z}, x^{\mu}\right)$ of the coordinates $x^{\mu}$ on $\mathcal{M}$ and of the ratio

$$
\mathbf{z} \equiv \frac{\bar{\pi}_{2} \zeta+\pi^{1}}{-\bar{\pi}_{1} \zeta+\pi^{2}}
$$

such that

$$
v_{[i]}\left(\zeta, \pi^{A^{\prime}}, \bar{\pi}_{A^{\prime}}, x^{\mu}\right)=f_{i 0}^{2 n}\left(\pi^{2}-\zeta \bar{\pi}_{1}\right)^{2 n} \tilde{v}_{[i]}\left(\mathbf{z}, x^{\mu}\right) .
$$

For anomalous $\mathcal{O}(0)$ sections, the same argument guarantees the existence of a function $\tilde{\mu}^{[i]}\left(\mathbf{z}, x^{\mu}\right)$ such that

$$
\mu^{[i]}\left(\zeta, \pi^{A^{\prime}}, \bar{\pi}_{A^{\prime}}, x^{\mu}\right)=\tilde{\mu}^{[i]}\left(\mathbf{z}, x^{\mu}\right)-c_{I}^{[i]} \log \left(f_{i 0}^{2}\left(\pi^{2}-\zeta \bar{\pi}_{1}\right)^{2}\right) .
$$


Moreover, under the action of the antipodal map,

$$
\overline{\tau(\mathbf{z})}=-1 / \mathbf{z}, \quad \overline{\tau\left(\tilde{v}_{[i]}\right)}=-\tilde{v}_{[\bar{i}]} / \mathbf{z}^{2 n}, \quad \overline{\tau\left(\tilde{\mu}^{[i]}\right)}=-\tilde{\mu}^{[\bar{l}]}-2 c^{[\bar{l}]} \log \mathbf{z} .
$$

The coordinate $\mathbf{z}$ can be viewed as a coordinate on the $\mathbb{C} P^{1}$ fiber of the twistor space $\mathcal{Z}_{\mathcal{M}}$. After an appropriate $S U(2)$ rotation on the $\mathbb{C}^{2} / \mathbb{Z}_{2}$ fiber, we can always assume that the zero and the pole of (2.57) occur at the zeros $\zeta_{ \pm}$of the singled-out section $v^{b}$,

$$
\mathbf{z}=-\frac{1}{\bar{z}} \frac{\zeta-\zeta_{+}}{\zeta-\zeta_{-}}, \quad \zeta_{+}=-\frac{\pi^{1}}{\bar{\pi}_{2}}, \quad \zeta_{-}=\frac{\pi^{2}}{\bar{\pi}_{1}}, \quad z=\frac{\pi^{1}}{\pi^{2}}
$$

In particular, the points $\left(0, \zeta_{+}, \zeta_{-}, \infty\right)$ in the $\zeta$ plane are mapped to $(z, 0, \infty,-1 / \bar{z})$ in the $\mathbf{z}$ plane, respectively. Since $\nu_{[i]}$ is assumed to be regular at $\zeta=\zeta_{i}, \tilde{v}_{[i]}\left(\mathbf{z}, x^{\mu}\right)$ is regular at the point $\mathbf{z}_{i} \equiv \mathbf{z}\left(\zeta_{i}\right)$, except when $i=-$, where the factors $\left(\pi^{2}-\zeta \bar{\pi}_{1}\right)$ introduce extra singularities at $\mathbf{z}=\infty$.

In the next subsection, we elaborate on these observations and relate the symplectic and contact structures on the twistor spaces $\mathcal{Z}_{\mathcal{S}}$ and $\mathcal{Z}_{\mathcal{M}}$.

2.5. Homogeneous symplectic vs. contact geometry. Having understood the constraints of superconformal invariance on the transition functions $S^{[i j]}$, we now explain how the homogeneous symplectic structure on $\mathcal{Z}_{\mathcal{S}}$ descends to a contact structure on $\mathcal{Z}_{\mathcal{M}}$, in effect rederiving the inverse construction of [13]. For definiteness, and since this is the case of most physical interest, we restrict to twistor spaces with $n=1$ from this section onward.

From homogeneous symplectic to contact. Let us return to (2.33): the term proportional to $\mathrm{d} \zeta$, usually unspecified in HK geometry, can be computed explicitly in the case of HKC manifolds. Indeed, by differentiating the factors $f_{i j}$ appearing explicitly in (2.52), and integrating by parts, one obtains

$$
\begin{aligned}
\mathrm{d}\left(S^{[i j]}-f_{i j}^{-2} \mu_{I}^{[i]} v_{[i]}^{I}\right)= & v_{[j]}^{I} \mathrm{~d} \mu_{I}^{[j]}-f_{i j}^{-2} v_{[i]}^{I} \mathrm{~d} \mu_{I}^{[i]} \\
& +\left(\hat{S}^{[i j]}+c_{I}^{[j]} \partial_{\mu_{I}^{[j]}} \hat{S}^{[i j]}-c_{I}^{[i]} v_{[i]}^{I} \log v_{[i]}^{\mathrm{b}}-\mu_{I}^{[i]} v_{[i]}^{I}\right) \mathrm{d}\left(f_{i j}^{-2}\right) .
\end{aligned}
$$

Re-expressing $\mu_{I}^{[i]}$ using (2.55) and using the homogeneity property of $\hat{S}^{[i j]}$, one concludes that

$$
\mathcal{X}_{[i]}=f_{i j}^{2} \mathcal{X}_{[j]}
$$

where $\mathcal{X}_{[i]}$ is the $\mathcal{O}(2)$-valued complex Liouville form

$$
\mathcal{X}_{[i]}=v_{[i]}^{I} \mathrm{~d} \mu_{I}^{[i]}+c_{I}^{[i]} \mathrm{d} v_{[i]}^{I},
$$

satisfying the reality condition $\overline{\tau\left(\mathcal{X}_{[i]}\right)}=\mathcal{X}_{[\bar{i}]}$. 
Let us now introduce the dilation-invariant $\mathcal{O}(0)$ sections $^{14}$

$$
\xi_{[i]}^{I} \equiv \frac{v_{[i]}^{I}}{v_{[i]}^{b}}, \quad \tilde{\xi}_{I}^{[i]} \equiv \mu_{I}^{[i]}+c_{I}^{[i]} \log v_{[i]}^{b},
$$

where we have singled out one coordinate ${ }^{15} v_{[i]}^{b}$, and denoted by $v_{[i]}^{\Lambda}$ the remaining $d$ coordinates. In this trivialization, the Liouville form $\mathcal{X}^{[i]}$ leads to a contact form $\hat{\mathcal{X}}^{[i]}$,

$$
\mathcal{X}^{[i]}=v_{[i]}^{b} \hat{\mathcal{X}}^{[i]}, \quad \hat{\mathcal{X}}^{[i]} \equiv \mathrm{d} \tilde{\xi}_{b}^{[i]}+\xi_{[i]}^{\Lambda} \mathrm{d} \tilde{\xi}_{\Lambda}^{[i]}+c_{\Lambda}^{[i]} \mathrm{d} \xi_{[i]}^{\Lambda} .
$$

The term linear in $c_{I}^{[i]}$ may be reabsorbed by defining

$$
\alpha^{[i]} \equiv \tilde{\xi}_{b}^{[i]}+c_{I}^{[i]} \xi_{[i]}^{I}, \quad \hat{\mathcal{X}}^{[i]}=\mathrm{d} \alpha^{[i]}+\xi_{[i]}^{\Lambda} \mathrm{d} \tilde{\xi}_{\Lambda}^{[i]} .
$$

The gluing condition (2.63) becomes

$$
\hat{\mathcal{X}}^{[i]}=\hat{f}_{i j}^{2} \hat{\mathcal{X}}^{[j]}, \quad \hat{f}_{i j}^{2} \equiv f_{i j}^{2} v_{[j]}^{b} / v_{[i]}^{b},
$$

while $\hat{\mathcal{X}}$ satisfies the reality condition $\overline{\tau\left(\hat{\mathcal{X}}^{[i]}\right)}=-\hat{\mathcal{X}}^{[\bar{i}]}$.

According to the remark at the end of the previous section, $\xi_{[i]}^{\Lambda}, \tilde{\xi}_{I}^{[i]}$ and $\hat{f}_{i j}^{2}$ are all functions of the $\mathbb{C} P^{1}$ coordinate $\mathbf{z}$ defined in (2.57) and of the coordinates $x^{\mu}$ on $\mathcal{M}$,

$$
\xi_{[i]}^{\Lambda}=\frac{\tilde{v}_{[i]}^{\Lambda}\left(x^{\mu}, \mathbf{z}\right)}{\tilde{v}_{[i]}^{b}\left(x^{\mu}, \mathbf{z}\right)}, \quad \tilde{\xi}_{I}^{[i]}=\tilde{\mu}_{I}^{[i]}\left(x^{\mu}, \mathbf{z}\right)+c_{I}^{[i]} \log \tilde{v}_{[i]}^{b}\left(x^{\mu}, \mathbf{z}\right), \quad \hat{f}_{i j}^{2}=\frac{\tilde{v}_{[j]}^{b}\left(x^{\mu}, \mathbf{z}\right)}{\tilde{v}_{[i]}^{b}\left(x^{\mu}, \mathbf{z}\right)} .
$$

Thus, the sections $\left(\xi_{[i]}^{\Lambda}, \tilde{\xi}_{I}^{[i]}\right)$ provide local complex Darboux coordinates for the complex contact structure $\hat{\mathcal{X}}$ on $\mathcal{Z}_{\mathcal{M}}$. They satisfy the reality conditions

$$
\overline{\tau\left(\xi_{[i]}^{\Lambda}\right)}=\xi_{[\bar{i}]}^{\Lambda}, \quad \overline{\tau\left(\tilde{\xi}_{I}^{[i]}\right)}=-\tilde{\xi}_{I}^{[\bar{i}]}+\mathrm{i} \pi c_{I}^{[\bar{l}]} .
$$

On the overlap of two patches, the Darboux coordinates are related by contact transformations following directly from (2.55),

$$
\begin{aligned}
& \xi_{[j]}^{\Lambda}=\hat{f}_{i j}^{-2} \partial_{\tilde{\xi}_{\Lambda}^{[j]}} \hat{S}^{[i j]}, \quad \tilde{\xi}_{\Lambda}^{[i]}=\partial_{\xi_{[i]}^{\Lambda}} \hat{S}^{[i j]}, \\
& \tilde{\xi}_{b}^{[i]}=\hat{S}^{[i j]}-\xi_{[i]}^{\Lambda} \partial_{\xi_{[i]}^{\Lambda}} \hat{S}^{[i j]}+c_{I}^{[j]} \partial_{\tilde{\xi}_{I}^{[j]}} \hat{S}^{[i j]}-c_{I}^{[i]} \xi_{[i]}^{I},
\end{aligned}
$$

where $\hat{S}^{[i j]}$ is a general function of $\xi_{[i]}^{\Lambda}$ and $\tilde{\xi}_{I}^{[j]}+c_{I}^{[j]} \log \hat{f}_{i j}^{-2}$, related to the original quasi-homogeneous generating function $S^{[i j]}$ via

$$
\begin{aligned}
S^{[i j]}\left(v_{[i]}^{I}, \mu_{I}^{[j]}, \zeta\right)= & f_{i j}^{-2}\left[v_{[i]}^{b} \hat{S}^{[i j]}\left(\xi_{[i]}^{\Lambda}, \tilde{\xi}_{I}^{[j]}+c_{I}^{[j]} \log \left(\hat{f}_{i j}^{-2}\right)\right)\right. \\
& \left.-c_{I}^{[i]} \xi_{[i]}^{I} v_{[i]}^{b} \log v_{[i]}^{b}\right],
\end{aligned}
$$

14 Our notations are related to the ones in [17] via $\xi^{\Lambda, N P V}=\xi_{[0]}^{\Lambda}, \tilde{\xi}_{\Lambda}^{\mathrm{NPV}}=-2 \mathrm{i} \tilde{\xi}_{\Lambda}^{[0]}, \alpha^{\mathrm{NPV}}=4 \mathrm{i} \tilde{\xi}_{b}^{[0]}+$ $2 \mathrm{i} \xi_{[0]}^{\Lambda} \tilde{\xi}_{\Lambda}^{[0]}$, where the quantities on the r.h.s. are evaluated at $\zeta=0, \mathbf{z}=z$.

15 Equation (2.65) is singular at the zeros of $v_{[i]}^{b}$, and one should in principle single out a second coordinate $v_{[i]}^{0}$ to cover these patches. Rather than doing so, we allow for poles and logarithmic cuts in $\xi_{[i]}^{\Lambda}$ and $\tilde{\xi}_{I}^{[i]}$, as in (2.80) below, in effect trivializing the $\mathcal{O}(2)$ bundle over $\mathbb{C} P_{\mathbf{z}}^{1}$. 
and it is understood that $\xi_{[i]}^{b}=1$. In particular, note that the transition functions $\hat{f}_{i j}^{2}$ are holomorphic functions on $\mathcal{Z}_{\mathcal{M}}$ given by

$$
\hat{f}_{i j}^{2}=\partial_{\tilde{\xi}_{b}^{[j]}} \hat{S}^{[i j]},
$$

and are equal to one if and only if $v^{b}$ is a global $\mathcal{O}(2)$ section.

Recovering the metric from the contact twistor lines. The functions $\xi_{[i]}^{\Lambda}\left(\mathbf{z}, x^{\mu}\right)$ and $\tilde{\xi}_{I}^{[i]}\left(\mathbf{z}, x^{\mu}\right)$ specify the twistor fiber over each point in $\mathcal{M}$, and are the analogs of the twistor lines $v_{[i]}^{I}(\zeta), \mu_{I}^{[i]}(\zeta)$ on $\mathcal{S}$. The knowledge of these "contact twistor lines" allows to reconstruct the Kähler-Einstein metric on $\mathcal{Z}_{\mathcal{M}}$ and the quaternionic-Kähler metric on $\mathcal{M}$, in the following manner.

First, identifying $\mathcal{X}^{[0]}=\mathcal{X}$ in (2.10) and using (2.58), the holomorphic contact form in any patch $\mathcal{U}_{i}$ may be written as

$$
\hat{\mathcal{X}}^{[i]}=\frac{\mathcal{X}^{[0]}}{f_{0 i}^{2} v_{[i]}^{b}}=\frac{1}{2}\left(\frac{\pi^{2}}{\pi^{2}-\zeta \bar{\pi}_{1}}\right)^{2} \frac{\mathrm{d} z+\mathcal{P}}{\tilde{v}_{[i]}^{b}\left(x^{\mu}, \mathbf{z}\right)},
$$

Since $\hat{\mathcal{X}}^{[i]}$ depends on the fiber coordinates $\pi^{A^{\prime}}, \bar{\pi}_{A^{\prime}}, \zeta$ only through the combination $\mathbf{z}$, we may set $\zeta=0, \mathbf{z}=z$ in this expression, and obtain

$$
\frac{\mathrm{d} \mathbf{z}}{\mathbf{z}}+\frac{p_{+}}{\mathbf{z}}-\mathrm{i} p_{3}+p_{-} \mathbf{z}=\frac{1}{2} e^{-\Phi_{[i]}} \hat{\mathcal{X}}^{[i]},
$$

where we define the "contact potential",

$$
e^{-\Phi_{[i]}\left(x^{\mu}, \mathbf{z}\right)} \equiv 4 \tilde{v}_{[i]}^{b}\left(x^{\mu}, \mathbf{z}\right) / \mathbf{z}, \quad \overline{\tau\left(\Phi_{[i]}\right)}=\Phi_{[\bar{i}]} .
$$

Applying (2.24), we conclude that the Kähler potential on $\mathcal{Z}_{\mathcal{M}}$ is given by

$$
K_{\mathcal{Z}_{\mathcal{M}}}=\log \frac{4(1+z \bar{z})}{|z|}+\operatorname{Re} \Phi_{[i]}\left(x^{\mu}, z\right)+\log \left|\hat{f}_{0 i}\right|^{2} .
$$

Since $\hat{f}_{0 i}$ is a holomorphic function, the last term in (2.77) can be absorbed by a Kähler transformation, leading to a Kähler potential valid in the patch $\mathcal{U}_{i}$. In order to derive the metric on $\mathcal{Z}$, one could therefore express $z, \bar{z}$ and $\Phi_{[i]}$ in terms of the complex coordinates $\left(\xi_{[i]}^{\Lambda}, \tilde{\xi}_{\Lambda}^{[i]}, \alpha^{[i]}\right)$ in $\hat{\mathcal{U}}_{i}$. For the purpose of computing the QK metric on $\mathcal{M}$, this step is unnecessary and it suffices to study the contact twistor lines, as we show below. For later reference, we record the hyperkähler potential which follows from (2.22) using $v^{b}=e^{2 \lambda}$

$$
\chi=4\left|v^{b} \hat{f}_{0 i}^{2}\right| \frac{1+z \bar{z}}{|z|} e^{\operatorname{Re}\left[\Phi_{[i]}\left(x^{\mu}, z\right)\right]} .
$$

By comparing (2.23) with (2.75) for $i=0$, we can also relate the coordinate $\psi$ in (2.14) to the coordinates $v^{b}, z, x^{\mu}$ on $\mathcal{S}$,

$$
e^{2 \mathrm{i} \psi}=\sqrt{\frac{v^{b} \bar{z}}{\bar{v}^{b} z}} e^{\mathrm{i} \operatorname{Im}\left[\Phi_{[0]}\left(x^{\mu}, z\right)\right]} .
$$


We now restrict our attention to the patches $\hat{\mathcal{U}}_{+}$and $\hat{\mathcal{U}}_{-}$around $\mathbf{z}=0$ and $\mathbf{z}=\infty$, respectively, corresponding to $\zeta=\zeta_{+}$and $\zeta=\zeta_{-}$. Using (2.65), (2.58), (2.59) and $f_{0+} \sim \zeta-\zeta_{-}$, we find that the contact twistor lines behave near $\mathbf{z}=0$ as

$$
\begin{aligned}
\xi_{[+]}^{\Lambda} & =\xi_{[+]}^{\Lambda,-1} \mathbf{z}^{-1}+\xi_{[+]}^{\Lambda, 0}+\xi_{[+]}^{\Lambda, 1} \mathbf{z}+\mathcal{O}\left(\mathbf{z}^{2}\right), \\
\tilde{\xi}_{\Lambda}^{[+]} & =c_{\Lambda}^{[+]} \log \mathbf{z}+\tilde{\xi}_{\Lambda, 0}^{[+]}+\tilde{\xi}_{\Lambda, 1}^{[+]} \mathbf{z}+\mathcal{O}\left(\mathbf{z}^{2}\right), \\
\alpha^{[+]} & =c_{b}^{[+]} \log \mathbf{z}+c_{\Lambda}^{[+]} \xi_{[+]}^{\Lambda,-1} \mathbf{z}^{-1}+\alpha_{0}^{[+]}+\alpha_{1}^{[+]} \mathbf{z}+\mathcal{O}\left(\mathbf{z}^{2}\right) .
\end{aligned}
$$

Similarly, near $\mathbf{z}=\infty$,

$$
\begin{aligned}
& \xi_{[-]}^{\Lambda}=\xi_{[-]}^{\Lambda,-1} \mathbf{z}+\xi_{[-]}^{\Lambda, 0}+\xi_{[-]}^{\Lambda, 1} \mathbf{z}^{-1}+\mathcal{O}\left(\mathbf{z}^{-2}\right), \\
& \xi_{\Lambda}^{[-]}=-c_{\Lambda}^{[-]} \log \mathbf{z}+\tilde{\xi}_{\Lambda, 0}^{[-]}+\tilde{\xi}_{\Lambda, 1}^{[-]} \mathbf{z}^{-1}+\mathcal{O}\left(\mathbf{z}^{-2}\right), \\
& \alpha^{[-]}=-c_{b}^{[-]} \log \mathbf{z}+c_{\Lambda}^{[-]} \xi_{[-]}^{\Lambda,-1} \mathbf{z}+\alpha_{0}^{[-]}+\alpha_{1}^{[-]} \mathbf{z}^{-1}+\mathcal{O}\left(\mathbf{z}^{-2}\right),
\end{aligned}
$$

where the Laurent coefficients are related to those at $\mathbf{z}=0$ by the reality conditions (2.70). It is also useful to specify the Laurent expansion of the contact potentials,

$$
\begin{aligned}
\Phi_{[+]} & =\phi_{[+]}^{0}+\phi_{[+]}^{1} \mathbf{z}+\mathcal{O}\left(\mathbf{z}^{2}\right), \\
\Phi_{[-]} & =\phi_{[-]}^{0}+\phi_{[-]}^{1} \mathbf{z}^{-1}+\mathcal{O}\left(\mathbf{z}^{-2}\right),
\end{aligned}
$$

related by the antipodal map $\Phi_{[-]}=\overline{\tau\left(\Phi_{[+]}\right)}$.

For generic choices of contact transformations, we expect ${ }^{16}$ that similarly to the HK case [22], the moduli space of solutions to the gluing conditions (2.71) and reality conditions (2.70) is of real dimension $4 d+1$, and can be parametrized by the lowest Laurent coefficients $\xi_{[+]}^{\Lambda,-1}=-\left(\xi_{[-]}^{\Lambda,-1}\right)^{*}, \tilde{\xi}_{\Lambda, 0}^{[+]}=-\left(\tilde{\xi}_{\Lambda, 0}^{[-]}\right)^{*}$ and the real coefficient $\mathrm{i}\left(\alpha_{0}^{[+]}+\alpha_{0}^{[-]}\right)$. This parameter space admits a $U(1)$ action induced by phase rotations of $\mathbf{z}$, which can be quotiented out to produce the $\mathrm{QK}$ manifold $\mathcal{M}$ itself.

Expanding the contact form (2.67) for $i= \pm$ at $\mathbf{z}=0, \infty$ and identifying the coefficients of $\mathbf{z}^{n}$ on either side of (2.75) allows to extract the $S U$ (2) connection,

$$
\begin{aligned}
p_{ \pm} & =\frac{1}{2} e^{-\phi_{[ \pm]}^{0}}\left(\xi_{[ \pm]}^{\Lambda,-1} \mathrm{~d} \tilde{\xi}_{\Lambda, 0}^{[ \pm]}+c_{\Lambda}^{[ \pm]} \mathrm{d} \xi_{[ \pm]}^{\Lambda,-1}\right), \\
p_{3} & =\frac{\mathrm{i}}{2} e^{-\phi_{[+]}^{0}}\left(\mathrm{~d} \alpha_{0}^{[+]}+\xi_{[+]}^{\Lambda, 0} \mathrm{~d} \tilde{\xi}_{\Lambda, 0}^{[+]}+\xi_{[+]}^{\Lambda,-1} \mathrm{~d} \tilde{\xi}_{\Lambda, 1}^{[+]}\right)-\mathrm{i} \phi_{[+]}^{1} p_{+},
\end{aligned}
$$

and to express the Laurent coefficients of the contact potentials in terms of the Laurent coefficients of the contact twistor lines,

$$
\begin{aligned}
e^{\phi_{[ \pm]}^{0}} & = \pm \frac{1}{2}\left(\xi_{[ \pm]}^{\Lambda,-1} \tilde{\xi}_{\Lambda, 1}^{[ \pm]}+c_{\Lambda}^{[ \pm]} \xi_{[ \pm]}^{\Lambda, 0}+c_{b}^{[ \pm]}\right), \\
\phi_{[ \pm]}^{1} & = \pm \frac{1}{2} e^{-\phi_{[ \pm]}^{0}}\left(\alpha_{1}^{[ \pm]}+2 \xi_{[ \pm]}^{\Lambda,-1} \tilde{\xi}_{\Lambda, 2}^{[ \pm]}+\xi_{[ \pm]}^{\Lambda, 0} \tilde{\xi}_{\Lambda, 1}^{[ \pm]}+c_{\Lambda}^{[ \pm]} \xi_{[ \pm]}^{\Lambda, 1}\right) .
\end{aligned}
$$

Via (2.1), one obtains the triplet of quaternionic forms $\vec{\omega}_{\mathcal{M}}$, in particular

$$
\omega_{\mathcal{M}, 3}=\frac{2}{v}\left(\mathrm{~d} p_{3}+2 \mathrm{i} p_{+} \wedge p_{-}\right) .
$$

\footnotetext{
${ }^{16}$ In the case where $\mathcal{M}$ admits $d+1$ commuting isometries, or for perturbations thereof, this will be demonstrated in Sects. 3 and 5 below.
} 
As anticipated above, the $U(1)$ action induced by phase rotations of $\mathbf{z}$ shifts $p_{3}$ by a total derivative and acts on $p_{ \pm}$in opposite ways, so lies in the kernel of $\omega_{3}$.

In order to obtain the metric from $\omega_{\mathcal{M}, 3}$, it is still necessary to specify the almost complex structure $J_{3}$. This is achieved by expanding the holomorphic one-forms $\mathrm{d} \xi_{[+]}^{\Lambda}$ and $\mathrm{d} \tilde{\xi}_{I}^{[+]}$around $\mathbf{z}=0$, and projecting them along the base $\mathcal{M}$ :

$$
\begin{aligned}
\mathrm{d} \xi_{[+]}^{\Lambda} & =\xi_{[+]}^{\Lambda,-1} p_{+} \mathbf{z}^{-2}+\mathcal{V}^{\Lambda} \mathbf{z}^{-1}+\mathcal{O}\left(\mathbf{z}^{0}\right) \quad \bmod D \mathbf{z}, \\
\mathrm{d} \tilde{\xi}_{I}^{[+]} & =-c_{I}^{[+]} p_{+} \mathbf{z}^{-1}+\tilde{\mathcal{V}}_{I}+\mathcal{O}\left(\mathbf{z}^{1}\right) \quad \bmod D \mathbf{z},
\end{aligned}
$$

where $D \mathbf{z}=d \mathbf{z}+p_{+}-\mathrm{i} p_{3} \mathbf{z}+p_{-} \mathbf{z}^{2}$ and

$$
\mathcal{V}^{\Lambda} \equiv\left(\mathrm{d}-\mathrm{i} p_{3}\right) \xi_{[+]}^{\Lambda,-1}, \quad \tilde{\mathcal{V}}_{I} \equiv \mathrm{d} \tilde{\xi}_{I, 0}^{[+]}-\tilde{\xi}_{I, 1}^{[+]} p_{+}+\mathrm{i} c_{I}^{[+]} p_{3}
$$

$(1,0)$ forms with respect to the almost complex structure $J_{3}$ on $\mathcal{M}$ can then be obtained by forming linear combinations of $\mathrm{d} \xi_{[+]}^{\Lambda}$ and $\mathrm{d} \tilde{\xi}_{I}^{[+]}$which are regular at $\mathbf{z}=0$, and setting $\mathbf{z}=0$ in the corresponding expressions. Thus, singling the index 0 out of $\Lambda$, a basis of $(1,0)$ forms on $\mathcal{M}$ is given by

$$
\Pi^{a}=\xi_{[+]}^{0,-1} \mathcal{V}^{a}-\xi_{[+]}^{a,-1} \mathcal{V}^{0}, \quad \tilde{\Pi}_{I}=\xi_{[+]}^{0,-1} \tilde{\mathcal{V}}_{I}+c_{I}^{[+]} \mathcal{V}^{0},
$$

where $a$ runs from 1 to $d-1$. Note that the $(1,0)$ form $p_{+}$is not linearly independent from those, as it satisfies

$$
\xi_{[+]}^{0,-1}\left(1-\xi_{[+]}^{\Lambda,-1} \tilde{\xi}_{\Lambda, 1}^{[+]}\right) p_{+}=\frac{1}{2} e^{-\phi_{[+]}^{0}}\left(\xi_{[+]}^{a,-1} \tilde{\Pi}_{a}+c_{\Lambda}^{[+]} \Pi^{\Lambda}\right)
$$

Having determined $J_{3}$ in this way, the QK metric then follows from $\omega_{3}(X, Y)=$ $g_{\mathcal{M}}\left(J_{3} X, Y\right)$. Of course, the $S U(2)$ connection and almost complex structure can equivalently be obtained by expanding near $\mathbf{z}=\infty$.

Before closing this section, let us note that the above discussion simplifies considerably in the special case where $v^{b}$ is a global $\mathcal{O}(2)$ section: in this case, the transition functions $\hat{f}_{i j}^{2}$ become equal to one, and the contact potentials $\Phi_{i}\left(x^{\mu}, \mathbf{z}\right)$ become independent of $\mathbf{z}$, defining a real function on $\mathcal{M}$.

\section{Quaternionic Geometry with Commuting Isometries}

In this section, we study aspects of the twistor space $\mathcal{Z}_{\mathcal{S}}$ of a HKC $\mathcal{S}$ (of real dimension $4 d+4$ ) with $d+1$ commuting tri-holomorphic isometries. As explained in the introduction, this situation arises when $\mathcal{S}$ is the Swann bundle of a QK manifold $\mathcal{M}$ with $d+1$ commuting isometries.

3.1. Tri-holomorphic isometries and superconformal invariance. As explained in [1], the moment maps associated to the $d+1$ commuting tri-holomorphic isometries provide $d+1$ global $\mathcal{O}(2)$ sections, which can be taken as the "position" coordinates $v^{I}$ $(I=b, 0, \ldots, d-1)$ for the holomorphic section $\Omega$. The fact that $v^{I}$ are global $\mathcal{O}(2)$ sections restricts the form of the transition functions $S^{[i j]}$ to

$$
S^{[i j]}\left(v_{[i]}, \mu^{[j]}, \zeta\right)=f_{i j}^{-2} v_{[i]}^{I} \mu_{I}^{[j]}-\tilde{H}^{[i j]}\left(v_{[i]}, \zeta\right),
$$


in such a way that, on the overlap of two patches,

$$
v_{[j]}^{I}=f_{i j}^{-2} v_{[i]}^{I}, \quad \mu_{I}^{[i]}=\mu_{I}^{[j]}-f_{i j}^{2} \partial_{v_{[i]}^{I}} \tilde{H}^{[i j]}\left(v_{[i]}, \zeta\right) .
$$

The condition of superconformal invariance (2.47) further restricts $\tilde{H}^{[i j]}\left(v_{[i]}, \zeta\right)$ to be of the form

$$
\tilde{H}^{[i j]}\left(v_{[i]}, \zeta\right)=f_{i j}^{-2} \hat{H}^{[i j]}\left(v_{[i]}\right),
$$

where $\hat{H}^{[i j]}\left(v_{[i]}\right)$ is a homogeneous function of degree one in $v_{[i]}{ }^{17}$. Following [1], we like to express $\tilde{H}^{[i j]}$ in terms of the standard $\mathcal{O}(2)$ multiplet

$$
\eta^{I}(\zeta) \equiv \zeta^{-1} v_{[0]}^{I}(\zeta)=\frac{v^{I}}{\zeta}+x^{I}-\bar{v}^{I} \zeta
$$

Thus, we define (cf. Eq. (3.7) in [1])

$$
H^{[i j]}\left(\eta^{I}, \zeta\right) \equiv \zeta^{-1} f_{0 j}^{2} \tilde{H}^{[i j]}\left(\zeta f_{0 i}^{-2} \eta, \zeta\right)
$$

Using (3.3), this reduces to

$$
H^{[i j]}\left(\eta^{I}, \zeta\right)=\hat{H}^{[i j]}\left(\eta^{I}\right) \equiv H^{[i j]}\left(\eta^{I}\right)
$$

In terms of $H^{[i j]}(\eta)$, the gluing conditions (3.2) simply become

$$
\mu_{I}^{[i]}=\mu_{I}^{[j]}-\partial_{\eta^{I}} H^{[i j]}(\eta) .
$$

The consistency conditions on $H^{[i j]}(\eta, \zeta)$ were analyzed in [1], and just need to be restricted to the superconformal case. Thus, we require that

$$
H^{[j i]}=-H^{[i j]}, \quad H^{[i k]}+H^{[k j]}=H^{[i j]},
$$

subject to the equivalence relation

$$
H^{[i j]} \mapsto H^{[i j]}+G^{[i]}-G^{[j]},
$$

and reality conditions

$$
\overline{\tau\left(H^{[i j]}\right)}=-H^{[\bar{\imath} \bar{\jmath}]},
$$

where all quantities are $\zeta$-independent functions of $\eta^{I}$, homogeneous of degree one. As in [1], we shall abuse notation and define $\hat{H}^{[i j]}$ away from the overlap $\hat{\mathcal{U}}_{i} \cap \hat{\mathcal{U}}_{j}$ (in particular when the two patches do not intersect) using analytic continuation and the second equation in (3.8) to interpolate from $\hat{\mathcal{U}}_{i}$ to $\hat{\mathcal{U}}_{j}$.

\footnotetext{
17 R. Ionas and A. Neitzke have independently shown that the condition that the generalized prepotential is a section of $\mathcal{O}(2)$ implies that $\mathcal{S}$ is HKC [27].
} 
Anomalous $\mathcal{O}(0)$ multiplets. As discussed in the previous section, it is possible to relax the homogeneity condition (2.47) into the "quasi-homogeneity" condition (2.52). In this case, $\tilde{H}^{[i j]}$ is restricted to be of the form

$$
\tilde{H}^{[i j]}\left(v_{[i]}, \zeta\right)=f_{i j}^{-2}\left[\hat{H}^{[i j]}\left(v_{[i]}\right)+c_{I}^{[i]} v_{[i]}^{I} \log v_{[i]}^{b}-c_{I}^{[j]} v_{[i]}^{I} \log \left(f_{i j}^{-2} v_{[i]}^{b}\right)\right],
$$

where $\hat{H}^{[i j]}\left(v_{[i]}\right)$ is again homogeneous of degree one in its argument. Defining $H^{[i j]}$ as in (3.5), we find

$$
H^{[i j]}(\eta, \zeta)=\hat{H}^{[i j]}(\eta)+c_{I}^{[i]} \eta^{I} \log \left(\zeta f_{0 i}^{-2} \eta^{b}\right)-c_{I}^{[j]} \eta^{I} \log \left(\zeta f_{0 j}^{-2} \eta^{b}\right) .
$$

The explicit dependence on $\zeta$ may be removed by a local symplectomorphism

$$
G^{[i]}=-c_{I}^{[i]} \eta^{I} \log \left(\zeta f_{0 i}^{-2}\right)+c_{I}^{[0]} \eta^{I} \log \zeta,
$$

where the second, $i$ independent term was added to ensure regularity in the patches $i=0$ and $i=\infty$. $^{18}$ After this gauge transformation, we find

$$
H^{[i j]}(\eta, \zeta)=\hat{H}^{[i j]}(\eta)+c_{I}^{[i j]} \eta^{I} \log \eta^{b} \equiv H^{[i j]}(\eta)
$$

where $c_{I}^{[i j]} \equiv c_{I}^{[i]}-c_{I}^{[j]}$, while the momentum coordinate $\mu^{[i]}$ is replaced by

$$
\mu_{T ; I}^{[i]}=\mu_{I}^{[i]}+c_{I}^{[i]} \log \left(\zeta f_{0 i}^{-2}\right)-c_{I}^{[0]} \log \zeta .
$$

Note that (3.14) is no longer a homogeneous function of $\eta^{I}$, but rather satisfies the quasi-homogeneity condition

$$
\left(\eta^{I} \partial_{\eta^{I}}-1\right) H^{[i j]}=c_{I}^{[i j]} \eta^{I}
$$

Complex contact structure. As in Sect. 2.5, using the (quasi)-homogeneity property of the transition functions $H^{[i j]}$, one may reduce the complex symplectic structure on $\mathcal{Z}_{\mathcal{S}}$ to a complex contact structure on $\mathcal{Z}_{\mathcal{M}}$. One should only be careful that due to the gauge transformation (3.13), the anomalous $\mathcal{O}(0)$ sections satisfy

$$
\mu_{T ; I}^{[i]}\left(\zeta, \pi^{A^{\prime}}, \bar{\pi}_{A^{\prime}}, x^{\mu}\right)=\tilde{\mu}_{T ; I}^{[i]}\left(\mathbf{z}, x^{\mu}\right)-c_{I}^{[i]} \log \left[\left(\pi^{2}-\zeta \bar{\pi}_{1}\right)^{2}\right]-c_{I}^{[0 i]} \log \zeta
$$

rather than (2.59), while $\tilde{\xi}_{I}^{[i]}\left(\mathbf{z}, x^{\mu}\right)$ defined in (2.65) becomes

$$
\tilde{\xi}_{I}^{[i]} \equiv \mu_{T ; I}^{[i]}(\zeta)+c_{I}^{[i]} \log \eta^{b}+c_{I}^{[0]} \log \zeta
$$

Using the fact that $\hat{f}_{i j}=1$ when $v^{b}$ is a globally defined $\mathcal{O}(2)$ section, the transition function (3.1) with $H^{[i j]}$ as in (3.14) then leads to

$$
\hat{S}^{[i j]}\left(\xi_{[i]}^{\Lambda}, \tilde{\xi}_{I}^{[j]}\right)=\tilde{\xi}_{b}^{[j]}+\xi_{[i]}^{\Lambda} \tilde{\xi}_{\Lambda}^{[j]}-\hat{H}^{[i j]}\left(\xi_{[i]}^{\Lambda}\right) .
$$

18 This is the place where the additional reality condition $c_{I}^{[0]}=-c_{I}^{[\infty]}$ becomes necessary. 
The section $\xi^{\Lambda} \equiv \xi_{[j]}^{\Lambda}$ is globally well defined, and therefore takes the form

$$
\xi^{\Lambda}=\mathbf{z}^{-1} Y_{+}^{\Lambda}+A^{\Lambda}-\mathbf{z} Y_{-}^{\Lambda},
$$

where $A^{\Lambda}$ is real and $\left(Y_{+}^{\Lambda}\right)^{*}=Y_{-}^{\Lambda}$. The vector $\left(2 \operatorname{Re}\left(Y_{+}^{\Lambda}\right), 2 \operatorname{Im}\left(Y_{+}^{\Lambda}\right), A^{\Lambda}\right)$ is in fact the generalized moment map for the translational isometry along $A^{\Lambda}$, as defined in [26]. The relation between $A^{\Lambda}, Y_{+}^{\Lambda}$ and $x^{I}, v^{I}$ will be discussed in Sect. 3.3 below.

On the other hand, the sections $\tilde{\xi}_{I}^{[i]}$ are defined only in the patch $\mathcal{U}_{i}$, and are related on the overlap of two patches by the complex contact transformation

$$
\begin{aligned}
& \tilde{\xi}_{\Lambda}^{[i]}=\tilde{\xi}_{\Lambda}^{[j]}-\partial_{\xi^{\Lambda}} \hat{H}^{[i j]}\left(\xi^{\Lambda}\right), \\
& \tilde{\xi}_{b}^{[i]}=\tilde{\xi}_{b}^{[j]}-\hat{H}^{[i j]}\left(\xi^{\Lambda}\right)+\xi^{\Lambda} \partial_{\xi^{\Lambda}} \hat{H}^{[i j]}\left(\xi^{\Lambda}\right)-c_{I}^{[i j]} \xi^{I} .
\end{aligned}
$$

It should be noted that the term proportional to $c_{\Lambda}^{[i j]}$ in this expression disappears when $\tilde{\xi}_{b}^{[i]}$ is traded for $\alpha^{[i]}$ as in (2.67),

$$
\alpha^{[i]}=\alpha^{[j]}-\hat{H}^{[i j]}\left(\xi^{\Lambda}\right)+\xi^{\Lambda} \partial_{\xi^{\Lambda}} \hat{H}^{[i j]}\left(\xi^{\Lambda}\right) .
$$

Lagrangian, hyperkähler potential and twistor lines. As explained in [1], the transition functions $H^{[i j]}(\eta)$ determine the holomorphic symplectic structure of $\mathcal{Z}_{\mathcal{S}}$, and therefore a HK metric on $\mathcal{S}$. The latter can be computed from the "Lagrangian", a function of the components $v^{I}, x^{I}, \bar{v}^{I}$ of $\eta^{I}$ defined by the contour integral

$$
\mathcal{L}=\sum_{j} \oint_{C_{j}} \frac{\mathrm{d} \zeta}{2 \pi \mathrm{i} \zeta} H^{[0 j]}(\eta(\zeta))
$$

where the contours $C_{j}$ encircle the centered disks $\mathcal{U}_{j}$ in the complex $\zeta$-plane. Note that due to the consistency conditions (3.8), the index 0 on the right-hand side of this expression may be substituted with any other value without changing the result. A Kähler potential for the HK metric on $\mathcal{S}$ is then obtained by Legendre transformation with respect to $x^{I}$ [22],

$$
\chi\left(v^{I}, w_{I}, \bar{v}^{I}, \bar{w}_{I}\right)=\mathcal{L}-x^{I} \partial_{x^{I}} \mathcal{L}, \quad \partial_{x^{I}} \mathcal{L}=w_{I}+\bar{w}_{I} .
$$

As shown in [1], the "momentum" coordinates $\mu_{I}^{[i]}(\zeta)$ are given by a single expression valid for all patches $i$,

$$
\mu_{T ; I}^{[i]}(\zeta)=\frac{\mathrm{i}}{2} \varrho_{I}+\sum_{j} \oint_{C_{j}} \frac{\mathrm{d} \zeta^{\prime}}{2 \pi \mathrm{i} \zeta^{\prime}} \frac{\zeta+\zeta^{\prime}}{2\left(\zeta^{\prime}-\zeta\right)} \partial_{\eta^{I}} H^{[0 j]}\left(\eta\left(\zeta^{\prime}\right)\right),
$$

provided $\zeta$ lies in the open disk $\mathcal{U}_{i}$. In particular, it is manifestly regular in $\mathcal{U}_{i}$. The coordinates $\varrho_{I} \equiv-\mathrm{i}\left(w_{I}-\bar{w}_{I}\right)$ correspond to overall additive constants unconstrained by (3.7), and are adapted to the tri-holomorphic isometries $\partial_{\varrho_{I}}$ of $\mathcal{S}$.

We now discuss the homogeneity and $S U(2)$ transformation properties of $\mathcal{L}$ and $\chi$. Taking into account the quasi-homogeneous property (3.16), we readily find the scaling relation

$$
\mathcal{L}\left(\Lambda^{2} v^{I}, \Lambda^{2} \bar{v}^{I}, \Lambda^{2} x^{I}\right)=\Lambda^{2}\left[\mathcal{L}\left(v^{I}, \bar{v}^{I}, x^{I}\right)-2 c_{I}^{[0]} x^{I} \log \Lambda^{2}\right] .
$$


On the other hand, the hyperkähler potential satisfies

$$
\chi\left(\Lambda^{2} v^{I}, \Lambda^{2} \bar{v}^{I}, w_{I}-c_{I}^{[0]} \log \Lambda^{2}, \bar{w}_{I}-c_{I}^{[0]} \log \Lambda^{2}\right)=\Lambda^{2} \chi\left(v^{I}, \bar{v}^{I}, w_{I}, \bar{w}_{I}\right) .
$$

The $S U$ (2) action on $v^{I}$ and $w_{I}$ can be obtained from (A.5) and (A.6), in Appendix A, leading to

$$
\begin{gathered}
\delta v^{I}=\mathrm{i} \epsilon_{3} v^{I}+\epsilon_{+} x^{I}, \quad \delta \bar{v}^{I}=-\mathrm{i} \epsilon_{3} \bar{v}^{I}+\epsilon_{-} x^{I}, \\
\delta w_{I}=\epsilon_{+} \mathcal{L}_{v^{I}}-\mathrm{i} \epsilon_{3} c_{I}^{[0]}, \quad \delta \bar{w}_{I}=\epsilon_{-} \mathcal{L}_{\bar{v}^{I}}+\mathrm{i} \epsilon_{3} c_{I}^{[0]},
\end{gathered}
$$

while the real combinations $x^{I}$ and $\varrho_{I}$ transform as

$$
\delta x^{I}=-2\left(\epsilon_{+} \bar{v}^{I}+\epsilon_{-} v^{I}\right), \quad \delta \varrho_{I}=-\mathrm{i}\left(\epsilon_{+} \mathcal{L}_{v^{I}}-\epsilon_{-} \mathcal{L}_{\bar{v}}\right)-2 \epsilon_{3} c_{I}^{[0]} .
$$

Note that in the quasi-homogeneous case, $w_{I}$ has anomalous transformations under dilations and $U(1)$ transformations [28], compared to the transformations found in [4]. The anomalous terms can be removed by defining $\hat{w}_{I} \equiv w_{I}+c_{I}^{[0]} \log v^{b}$.

It is instructive to check explicitly that $\chi$ is $S U(2)$ invariant. Keeping only the homogeneous term in (3.14), one may rewrite

$$
\begin{aligned}
\hat{\chi}= & \sum_{j} \oint_{C_{j}} \frac{\mathrm{d} \zeta}{2 \pi \mathrm{i} \zeta}\left[\hat{H}^{[0 j]}\left(\eta^{I}\right)-x^{I} \partial_{\eta^{I}} \hat{H}^{[0 j]}\left(\eta^{I}\right)\right] \\
= & \sum_{j} \oint_{C_{j}} \frac{\mathrm{d} \zeta}{2 \pi \mathrm{i} \zeta}\left[\left(\frac{v^{b}}{\zeta}-\bar{v}^{b} \zeta\right) \frac{\hat{H}^{[0 j]}}{\eta^{b}}\right. \\
& \left.+\left(\frac{v^{\Lambda} x^{b}-x^{\Lambda} v^{b}}{\zeta}+\left(x^{\Lambda} \bar{v}^{b}-\bar{v}^{\Lambda} x^{b}\right) \zeta\right) \frac{\partial_{\eta^{\Lambda}} \hat{H}^{[0 j]}}{\eta^{b}}\right] .
\end{aligned}
$$

Integrating the round bracket in the first term by parts, a short computation establishes

$$
\hat{\chi}=\left(r^{b}\right)^{2} \sum_{j} \oint_{C_{j}} \frac{\mathrm{d} \zeta}{2 \pi \mathrm{i} \zeta} \frac{\eta^{\Lambda} \partial_{\eta^{\Lambda}} \hat{H}^{[0 j]}}{\left(\eta^{b}\right)^{2}}-\left(\vec{r}^{b} \cdot \vec{r}^{\Lambda}\right) \sum_{j} \oint_{C_{j}} \frac{\mathrm{d} \zeta}{2 \pi \mathrm{i} \zeta} \frac{\partial_{\eta^{\Lambda}} \hat{H}^{[0 j]}}{\eta^{\mathrm{b}}}
$$

where

$$
\vec{r}^{I} \cdot \vec{r}^{J}=x^{I} x^{J}+2 v^{I} \bar{v}^{J}+2 \bar{v}^{I} v^{J}
$$

is the inner product of the 3 -vectors $\vec{r}^{I}=\left(2 \operatorname{Re}\left(v^{I}\right), 2 \operatorname{Im}\left(v^{I}\right), x^{I}\right)$ associated to the $\mathcal{O}(2)$ multiplets $\eta^{I}$. Each term is the product of a $S U(2)$ invariant quantity times a contour integral of an $\mathcal{O}(-2)$ section, and so (according to a general argument discussed at the end of Appendix A) is $S U$ (2) invariant. In the quasi-homogeneous case, the same line of argument combined with the contour deformations discussed in Sect. 3.4 of [1] leads to

$$
\chi=\hat{\chi}+c_{I}^{[+-]} \frac{\vec{r}^{b} \cdot \vec{r}^{I}}{r^{b}},
$$

where $c_{I}^{[+-]}$denotes the quasi-homogeneity coefficient relating the patches around the two roots of $\eta^{b}(\zeta)$. Thus $\chi$ is $S U(2)$ invariant, and therefore equal to the hyperkähler potential on $\mathcal{S}$. This concludes the proof that transition functions of the form (3.14) indeed lead to a HKC metric on $\mathcal{S}$. 
3.2. Superconformal quotient. In this subsection and the following one, we perform the superconformal quotient explicitly for a general $\mathrm{HKC} \mathcal{S}$ described by the formalism of the previous subsection. We start by constructing a convenient set of coordinates $x^{\mu}, \pi^{A^{\prime}}, \bar{\pi}_{A^{\prime}}$ on $\mathcal{M} \times \mathbb{C}^{2} / \mathbb{Z}^{2}$, in terms of the complex coordinates $v^{I}(\zeta), \mu_{I}(\zeta)$ on the Swann bundle $\mathcal{S}$ in an arbitrary complex structure $\zeta$. In the next subsection, we find the reciprocal change of variables, and determine the twistor lines.

The real coordinates $x^{\mu}$ on $\mathcal{M}$ are characterized by their invariance under the scaling and isometric $S U(2)$ actions on $\mathcal{Z}_{\mathcal{S}}$. Instead, the coordinates $\pi^{A^{\prime}}, \bar{\pi}_{A^{\prime}}$ should transform as a pair of doublets under $S U(2)$, and have a squared norm dictated by the hyperkähler potential, $\pi^{A^{\prime}} \bar{\pi}_{A^{\prime}}=\chi$. These constraints do not determine the coordinates $x^{\mu}, \pi^{A^{\prime}}, \bar{\pi}_{A^{\prime}}$ uniquely. In the case of QK spaces obtained by the classical and quantum corrected $c$-map, studied in $[17,28]$, it was convenient to choose coordinates $x^{\mu}$ adapted to the action of a $2 d+1$ dimensional Heisenberg group of isometries. In the general $\mathcal{O}(2)$ case, the only isometries are the $d+1$ abelian shift symmetries, and there is no such "canonical" choice. Our construction below is tailored to reproduce the results $[17,28]$ for $c$-map spaces, as we illustrate later in Sect. 4. It also follows from considerations in contact geometry, as discussed in greater generality in Sect. 5.2.

We start by singling out two multiplets $\eta^{\mathrm{b}}, \eta^{0}$ of the $d+1 \mathcal{O}(2)$ multiplets $\eta^{I}$, and denote by $\eta^{a}, a=1, \ldots, d-1$, the remaining ones. The zeros of $\nu_{[0]}^{b}=\zeta \eta^{b}$ are now

$$
\zeta_{ \pm}=\frac{x^{b} \mp r^{b}}{2 \bar{v}^{b}}, \quad r^{b}=\sqrt{\left(x^{b}\right)^{2}+4 v^{b} \bar{v}^{b}}
$$

As explained below (A.9), $S U(2)$-invariant quantities can be constructed by contourintegrating $\mathcal{O}(-2)$ sections on $\mathcal{S}$. The simplest example is

$$
\oint_{C_{+}} \frac{\mathrm{d} \zeta}{2 \pi \mathrm{i} \zeta} \frac{1}{\eta^{b}}=\frac{1}{r^{b}}
$$

which recovers the $S U(2)$ invariant $r^{b}$, homogeneous of degree one under dilations. Other convenient $S U(2)$ and dilation invariants are given by

$$
\begin{aligned}
A^{I} & \equiv r^{b} \oint_{C_{+}} \frac{\mathrm{d} \zeta}{2 \pi \mathrm{i} \zeta} \frac{\eta^{I}}{\left(\eta^{\mathrm{b}}\right)^{2}}=\frac{\left(\vec{r}^{\mathrm{b}} \cdot \vec{r}^{I}\right)}{\left(r^{\mathrm{b}}\right)^{2}} \\
Z^{\Lambda} & \equiv r^{\mathrm{b}} \oint_{C_{+}} \frac{\mathrm{d} \zeta}{2 \pi \mathrm{i} \zeta} \frac{\eta^{\Lambda}}{\eta^{b} \eta^{0}}=\frac{\eta_{+}^{\Lambda}}{\eta_{+}^{0}}, \quad \bar{Z}^{\Lambda} \equiv-r^{b} \oint_{C_{-}} \frac{\mathrm{d} \zeta}{2 \pi \mathrm{i} \zeta} \frac{\eta^{\Lambda}}{\eta^{b} \eta^{0}}=\frac{\eta_{-}^{\Lambda}}{\eta_{-}^{0}}
\end{aligned}
$$

and, when $\mu^{[i]}$ is a non-anomalous $\mathcal{O}(0)$ local section,

$$
B_{I} \equiv-\mathrm{i} r^{\mathrm{b}} \oint_{C_{+}} \frac{\mathrm{d} \zeta}{2 \pi \mathrm{i} \zeta} \frac{\mu_{I}^{[+]}}{\eta^{\mathrm{b}}}+\mathrm{i} r^{\mathrm{b}} \oint_{C_{-}} \frac{\mathrm{d} \zeta}{2 \pi \mathrm{i} \zeta} \frac{\mu_{I}^{[-]}}{\eta^{\mathrm{b}}}=-\mathrm{i}\left(\mu_{I}^{+}+\mu_{I}^{-}\right)
$$

Here $C_{ \pm}$denote the contours containing $\zeta_{ \pm}, \mu_{I}^{[ \pm]}$are the multiplets which are regular in the patch containing $\zeta_{ \pm}, \eta_{ \pm}^{\Lambda}=\eta^{\Lambda}\left(\zeta_{ \pm}\right)$, and $\mu_{I}^{ \pm}=\mu_{I}^{[ \pm]}\left(\zeta_{ \pm}\right)$. Moreover, an additional invariant can be constructed out of the hyperkähler potential itself,

$$
e^{\phi} \equiv \frac{\chi}{4 r^{b}}
$$


As we shall see the $4 d$ variables $x^{\mu}=\left\{\phi, Z^{a}, \bar{Z}^{a}, A^{\Lambda}, B_{I}\right\}$ provide a convenient coordinate system on $\mathcal{M}$. In particular, the coordinates $B_{I}$ correspond to the directions along the $d+1$ isometries.

In the quasi-homogeneous case, (3.37), is no longer $S U$ (2)-invariant. One may imagine replacing $\mu_{I}^{[+]}$by $\tilde{\xi}_{I}^{[+]}$, which is non-anomalous, however this quantity is singular in the patch $\mathcal{U}_{+}$, as apparent from (2.80). The logarithmic singularity can be cancelled without affecting the $S U(2)$ transformation properties by adding $c_{I}^{[+]} \log \left(\eta^{0} / \eta^{\mathrm{b}}\right)$, which leads us to define ${ }^{19}$

$$
B_{I} \equiv-\mathrm{i} r^{b} \oint_{C_{+}} \frac{\mathrm{d} \zeta}{2 \pi \mathrm{i} \zeta \eta^{b}}\left(\mu_{T ; I}^{[+]}+c_{I}^{[+]} \log \eta^{0}+c_{I}^{[0]} \log \zeta\right)-(+\leftrightarrow-) .
$$

It is important to note that there exists another manifestly $S U(2)$ and dilation invariant quantity,

$$
\mathcal{R} \equiv \frac{\left|\vec{r}^{b} \times \vec{r}^{0}\right|}{2\left(r^{b}\right)^{2}}=\frac{\left|v^{b} \eta_{+}^{0}\right|}{\left(r^{b}\right)^{2}}
$$

where $\times$ denotes the inner product of vectors in $\mathbb{R}^{3}$. As we shall see shortly, it is $\mathcal{R}$ rather than $\phi$ which appears most naturally in the general formulae (3.50) for the twistor lines. Note that $\mathcal{R}$ vanishes when the zeros of $\eta^{b}$ and $\eta^{0}$ collide.

As far as the coordinates on the fiber $\pi^{A^{\prime}}, \bar{\pi}_{A^{\prime}}$ are concerned, the reasoning below (A.9) shows that $S U(2)$ doublets can be constructed by contour-integrating $\mathcal{O}(-3)$ sections. Thus, it is natural to consider ${ }^{20}$

$$
\begin{aligned}
\pi^{1} & =C \oint_{\mathcal{C}_{+}} \frac{\mathrm{d} \zeta}{2 \pi \mathrm{i}} \frac{1}{\eta^{\mathrm{b}} \sqrt{\zeta \eta^{0}}}=\frac{C}{r^{b}} \sqrt{\frac{\zeta_{+}}{\eta_{+}^{0}}}, \\
\pi^{2} & =\bar{C} \oint_{\mathcal{C}_{-}} \frac{\mathrm{d} \zeta}{2 \pi \mathrm{i}} \frac{1}{\eta^{\mathrm{b}} \sqrt{-\zeta \eta^{0}}}=-\frac{\bar{C}}{r^{b}} \sqrt{-\frac{\zeta_{-}}{\eta_{-}^{0}}}, \\
\bar{\pi}_{1} & =-\bar{C} \oint_{\mathcal{C}_{-}} \frac{\mathrm{d} \zeta}{2 \pi \mathrm{i}} \frac{1}{\zeta \eta^{b} \sqrt{-\zeta \eta^{0}}}=\frac{\bar{C}}{r^{\mathrm{b}} \sqrt{-\zeta_{-} \eta_{-}^{0}}}, \\
\bar{\pi}_{2} & =-C \oint_{\mathcal{C}_{+}} \frac{\mathrm{d} \zeta}{2 \pi \mathrm{i}} \frac{1}{\zeta \eta^{b} \sqrt{\zeta \eta^{0}}}=-\frac{C}{r^{\mathrm{b}} \sqrt{\zeta_{+} \eta_{+}^{0}}},
\end{aligned}
$$

where the proportionality constant $C$ can be chosen to be real, and adjusted such that (2.20) is obeyed. This gives the $S U(2)$ invariant

$$
C=2 r^{b} e^{\phi / 2} \sqrt{\left|v^{b} \eta_{+}^{0}\right|}
$$

Equation (2.14) may now be rewritten as in [17],

$$
\left(\begin{array}{c}
\pi^{1} \\
\pi^{2}
\end{array}\right)=2 \mathrm{e}^{\phi / 2} \sqrt{v^{b}}\left(\begin{array}{c}
z^{\frac{1}{2}} \\
z^{-\frac{1}{2}}
\end{array}\right), \quad z=\zeta_{+} \sqrt{\frac{\bar{v}^{b} \bar{\eta}_{+}^{0}}{v^{b} \eta_{+}^{0}}}, \quad e^{4 \mathrm{i} \psi}=\frac{v^{b}}{\bar{v}^{b}} \frac{\bar{z}}{z} .
$$

19 This definition arises naturally from the general procedure explained in Sect. 5.2.

20 The contour integrals given below suffer from ambiguities in the choice of square root branches. This is inherent to the fact that the fiber of the Swann bundle is $\mathbb{C}^{2} / \mathbb{Z}_{2}$. We choose the branch cuts in such a way that the reality condition $\bar{\pi}_{A^{\prime}}=\left(\pi^{A^{\prime}}\right)^{*}$ is obeyed, and $\left(\pi^{A^{\prime}}, \epsilon^{A^{\prime} B^{\prime}} \bar{\pi}_{B^{\prime}}\right)$ transform as $S U$ (2) doublets. 
The following further relations are also often useful:

$$
\zeta_{-}=\frac{1}{|z|} \sqrt{\frac{v^{b}}{\bar{v}^{b}}}, \quad \zeta_{+}=-|z| \sqrt{\frac{v^{b}}{\bar{v}^{b}}}, \quad r^{b}=\frac{\left|v^{b}\right|}{|z|}(1+z \bar{z}), \quad x^{b}=\frac{\left|v^{b}\right|}{|z|}(1-z \bar{z}) .
$$

Finally, we note that the reduced $\mathcal{O}(2)$ global section $\tilde{v}_{[i]}^{b}$ defined in (2.58) takes the simple form

$$
\tilde{v}_{[i]}^{b}=\frac{1}{4} \mathrm{e}^{-\phi} \mathbf{z}
$$

Comparing with (2.76), we see that the contact potential $\Phi_{[+]}$is real, and coincides with the invariant $\phi$ defined in (3.38). This is also apparent from (2.78), using the third equation in (3.44) and the fact that $\hat{f}_{i j}=1 \mathrm{in} \mathcal{O}(2)$ geometries.

3.3. Contact twistor lines. We now consider the converse problem, of determining the complex coordinates $v^{I}, \mu_{I}$ on $\mathcal{S}$ in terms of the coordinates $x^{\mu}$ on the QK base $\mathcal{M}$ and of the coordinates $\pi^{A^{\prime}}$ on the $\mathbb{C}^{2}$ fiber. This is known in mathematics as "parametrizing the twistor lines". In view of the discussion in Sect. 2.5, this is equivalent to expressing the "contact twistor lines" $\left(\xi^{\Lambda}, \tilde{\xi}_{I}^{[i]}\right)$ in terms of the coordinates $\left(\mathbf{z}, x^{\mu}\right)$ on $\mathcal{Z}_{\mathcal{M}}$.

Let us consider first $\xi^{\Lambda}\left(\mathbf{z}, x^{\mu}\right)$. As explained in (2.80), $\xi^{\Lambda}$ (equal to $\xi_{[i]}^{\Lambda}$ for any $i$ ) admits a single pole at $\mathbf{z}=0$ and $\mathbf{z}=\infty$. The coefficients of the Laurent expansion of $\xi^{\Lambda}\left(\mathbf{z}, x^{\mu}\right)$ around $\mathbf{z}=0$ can be extracted from the contour integral

$$
\xi^{\Lambda, k}=\oint_{0} \frac{d \mathbf{z}}{2 \pi \mathbf{i} \mathbf{z}^{k+1}} \xi^{\Lambda}\left(\mathbf{z}, x^{\mu}\right)=r^{b} \oint_{C_{+}} \frac{d \zeta}{2 \pi \mathrm{i} \zeta} \frac{\eta^{\Lambda}}{\left(\eta^{b}\right)^{2}} \mathbf{z}^{-k},
$$

where we used

$$
\frac{\mathrm{d} \mathbf{z}}{2 \pi \mathrm{i} \mathbf{z}}=r^{b} \frac{\mathrm{d} \zeta}{2 \pi \mathrm{i} \zeta \eta^{b}}
$$

Equation (3.46) vanishes for $k \leq-2$ and $k \geq 2$, as can be seen by deforming the contour around $\zeta_{+}$to a contour around $\zeta_{-}$. For $k=0$, one immediately recovers the first quantity in (3.36). For $k=-1$, one may decompose

$$
\xi^{\Lambda,-1}=r^{b} \oint_{C_{+}} \frac{d \zeta}{2 \pi \mathrm{i} \zeta} \frac{\eta^{\Lambda}}{\eta^{b} \eta^{0}}\left[\frac{\mathbf{z} \eta^{0}}{\eta^{b}}\right]=\mathcal{R} Z^{\Lambda},
$$

where $Z^{0} \equiv 1$, and we used the fact that the term in brackets is regular at $\zeta=\zeta_{+}$and equal at that point to the invariant $\mathcal{R}$ defined in (3.40). For $k=1$, a similar argument leads to $\xi^{\Lambda, 1}=-\mathcal{R} \bar{Z}^{\Lambda}$. As a side product, we obtain a contour integral representation for the quantity (3.40),

$$
\mathcal{R}=r^{b} \oint_{C_{+}} \frac{\mathrm{d} \zeta}{2 \pi \mathrm{i} \zeta} \frac{\eta^{0}}{\left(\eta^{b}\right)^{2}} \mathbf{z}=r^{\mathrm{b}} \oint_{C_{-}} \frac{\mathrm{d} \zeta}{2 \pi \mathrm{i} \zeta} \frac{\eta^{0}}{\left(\eta^{b}\right)^{2}} \mathbf{z}^{-1}
$$

Thus, we conclude that the contact twistor line is parametrized by

$$
\xi^{\Lambda}\left(\mathbf{z}, x^{\mu}\right)=A^{\Lambda}+\mathcal{R}\left(\mathbf{z}^{-1} Z^{\Lambda}-\mathbf{z} \bar{Z}^{\Lambda}\right),
$$


so that $Y_{+}^{\Lambda}=\mathcal{R} Z^{\Lambda}$ in (3.20). Setting $\zeta=0, \mathbf{z}=z$ in this expression allows to express the complex coordinate $\xi^{\Lambda}$ on $\mathcal{Z}_{\mathcal{M}}$ in terms of the coordinates on the base and on the $\mathbb{C} P^{1}$ fiber.

For $\tilde{\xi}_{I}^{[i]}$ defined in (2.65), and assuming $c_{I}^{[i]}=0$ for simplicity, one may eliminate $\varrho_{I}$ in (3.25) in favor of $B_{I}$, and use the identity

$$
\frac{\mathrm{d} \zeta^{\prime}}{2 \pi \mathrm{i} \zeta^{\prime}}\left[\frac{\zeta+\zeta^{\prime}}{\zeta^{\prime}-\zeta}-\frac{1}{2} \frac{\zeta_{+}+\zeta^{\prime}}{\zeta^{\prime}-\zeta_{+}}-\frac{1}{2} \frac{\zeta_{-}+\zeta^{\prime}}{\zeta^{\prime}-\zeta_{-}}\right]=\frac{d \mathbf{z}^{\prime}}{2 \pi \mathrm{i} \mathbf{z}^{\prime}} \frac{\mathbf{z}+\mathbf{z}^{\prime}}{\mathbf{z}^{\prime}-\mathbf{z}}
$$

where $\mathbf{z}^{\prime}$ is obtained from (3.47) by replacing $\zeta \rightarrow \zeta^{\prime}, \mathbf{z} \rightarrow \mathbf{z}^{\prime}$. This gives

$$
\tilde{\xi}_{I}^{[0]}\left(\mathbf{z}, x^{\mu}\right)=\frac{\mathrm{i}}{2} B_{I}+\frac{1}{2} \sum_{j} \oint_{\tilde{C}_{j}} \frac{d \mathbf{z}^{\prime}}{2 \pi \mathrm{i} \mathbf{z}^{\prime}} \frac{\mathbf{z}^{\prime}+\mathbf{z}}{\mathbf{z}^{\prime}-\mathbf{z}} H_{I}^{[0 j]}\left(\xi\left(\mathbf{z}^{\prime}\right)\right),
$$

where $\tilde{C}_{i}$ is the image of the contour $C_{i}$ in the $\mathbf{z}^{\prime}$ plane, and $H_{I}^{[0 j]}(\xi) \equiv \partial_{\eta^{I}} H^{[0 j]}\left(\eta^{I}\right)$. In the quasi-homogeneous case, similar manipulations (explained in greater generality in Sect. 5.2) lead to

$$
\begin{aligned}
& \tilde{\xi}_{\Lambda}^{[0]}=\frac{\mathrm{i}}{2} B_{\Lambda}+\frac{1}{2} \sum_{j} \oint_{\tilde{C}_{j}} \frac{d \mathbf{z}^{\prime}}{2 \pi \mathrm{i} \mathbf{z}^{\prime}} \frac{\mathbf{z}^{\prime}+\mathbf{z}}{\mathbf{z}^{\prime}-\mathbf{z}} \partial_{\xi^{\Lambda}} \hat{H}^{[0 j]}\left(\xi\left(\mathbf{z}^{\prime}\right)\right)+\frac{1}{2} c_{\Lambda}^{[+-]} \log \mathbf{z}, \\
& \tilde{\xi}_{b}^{[0]}=\frac{\mathrm{i}}{2} B_{b}+\frac{1}{2} \sum_{j} \oint_{\tilde{C}_{j}} \frac{d \mathbf{z}^{\prime}}{2 \pi \mathrm{i} \mathbf{z}^{\prime}} \frac{\mathbf{z}^{\prime}+\mathbf{z}}{\mathbf{z}^{\prime}-\mathbf{z}}\left[\hat{H}-\xi^{\Lambda} \partial_{\xi^{\Lambda}} \hat{H}+c_{I} \xi^{I}\right]^{[0 j]}+\frac{1}{2} c_{b}^{[+-]} \log \mathbf{z} .
\end{aligned}
$$

Again, by substituting $\zeta=0, \mathbf{z}=z$ in these expressions, one may obtain the complex coordinate $\tilde{\xi}_{I}$ on $\mathcal{Z}_{\mathcal{M}}$ in terms of the fiber coordinate $z$ and the coordinates on $\mathcal{M}$. As in the case of (3.25), the r.h.s. of (3.53) gives the contact twistor line $\tilde{\xi}_{I}^{[i]}$ in any patch $\mathcal{U}_{i}$, provided $\mathbf{z}$ is chosen to lie in the corresponding patch (moreover, as in (3.25), one may replace the superscript $[0 j]$ with any $[k j]$ without changing the result). Indeed, one may check that the discontinuity of the r.h.s. of (3.53) across the contours $\tilde{C}_{i}$ precisely implements the contact transformations given in (3.21). Another important remark is that, due to the fact that the argument $\xi^{\Lambda}$ of $\hat{H}^{[0 j]}$ has a pole at $\mathbf{z}^{\prime}=0$, the integrals appearing in (3.53) need not be regular at $\mathbf{z}=0$ : we shall see an example of this phenomenon in (4.23) below.

For what concerns $\tilde{\xi}_{I}^{[+]}$however, the integrals are regular and the first Laurent coefficients needed to find the $S U$ (2) connection and the quaternionic-Kähler metric are readily extracted:

$$
\begin{gathered}
\tilde{\xi}_{\Lambda, 0}^{[+]}=\frac{\mathrm{i}}{2} B_{\Lambda}+\frac{1}{2} \sum_{j} \oint_{\tilde{C}_{j}} \frac{d \mathbf{z}}{2 \pi \mathrm{i} \mathbf{z}} \partial_{\xi \Lambda} \hat{H}^{[0 j]}, \quad \tilde{\xi}_{\Lambda, 1}^{[+]}=\sum_{j} \oint_{\tilde{C}_{j}} \frac{d \mathbf{z}}{2 \pi \mathrm{i} \mathbf{z}^{2}} \partial_{\xi^{\Lambda}} \hat{H}^{[0 j]} \\
\alpha_{0}^{[+]}=\frac{\mathrm{i}}{2} B_{b}+\frac{1}{2} \sum_{j} \oint_{\tilde{C}_{j}} \frac{d \mathbf{z}^{\prime}}{2 \pi \mathrm{i} \mathbf{z}^{\prime}}\left(\hat{H}^{[0 j]}-\xi^{\Lambda} \partial_{\xi^{\Lambda}} \hat{H}^{[0 j]}\right) .
\end{gathered}
$$


From these expressions it is easy to find the contact potentials using (2.84),

$$
\begin{aligned}
e^{\Phi_{[+]}} & =\frac{1}{2} \mathcal{R} \sum_{j} \oint_{\tilde{C}_{j}} \frac{d \mathbf{z}}{2 \pi \mathrm{i} \mathbf{z}^{2}} Z^{\Lambda} \partial_{\xi^{\Lambda}} \hat{H}^{[0 j]}\left(\xi^{\Lambda}(\mathbf{z})\right)+\frac{1}{2} c_{I}^{[+]} A^{I}, \\
e^{\Phi_{[-]}} & =-\frac{1}{2} \mathcal{R} \sum_{j} \oint_{\tilde{C}_{j}} \frac{d \mathbf{z}}{2 \pi \mathrm{i}} \bar{Z}^{\Lambda} \partial_{\xi^{\Lambda}} \hat{H}^{[0 j]}\left(\xi^{\Lambda}(\mathbf{z})\right)-\frac{1}{2} c_{I}^{[-]} A^{I},
\end{aligned}
$$

In particular, $e^{\Phi_{[ \pm]}}$are independent of the fiber coordinate $\mathbf{z}$ and are equal to each other, since their difference is the integral of a total derivative.

On the other hand, changing variable from $\zeta^{\prime}$ to $\mathbf{z}^{\prime}$ in (3.31), we may rewrite the hyperkähler potential $\chi$, Eq. (3.33), as a contour integral

$$
\chi=r^{\mathrm{b}} \mathcal{R} \sum_{j} \oint_{\tilde{C}_{j}} \frac{d \mathbf{z}}{2 \pi \mathrm{i} \mathbf{z}}\left(\mathbf{z}^{-1} Z^{\Lambda}-\mathbf{z} \bar{Z}^{\Lambda}\right) \partial_{\xi^{\Lambda}} \hat{H}^{[0 j]}\left(\xi^{\Lambda}(\mathbf{z})\right)+r^{\mathrm{b}} c_{I}^{[+-]} A^{I},
$$

where we used the notation $A^{b}=1$. Equation (3.56) may be used to express $\mathcal{R}$ in terms of $e^{\phi}$ or vice-versa. Moreover, comparing (3.56) and (3.55), one confirms that the invariant $\phi$ defined in (3.38) is indeed equal to the contact potential $\Phi_{[ \pm]}$.

\section{The Perturbative Hypermultiplet Moduli Space}

In order to illustrate the results in the previous section, we now discuss the geometry of hypermultiplet moduli spaces in type II string theories compactified on a Calabi-Yau three-fold, which is the main motivation for this study. In Sect. 4.1 we focus on the tree-level geometry, deferring the inclusion of the one-loop correction to the next subsection. Non-perturbative contributions will be considered in [19], using the results on deformation in Sect. 5 below.

4.1. Tree-level geometry. At tree-level in the string perturbative expansion, the hypermultiplet moduli space $\mathcal{M}$ in type IIA (resp. IIB) string theory compactified on a Calabi-Yau three-fold $Y$ (resp. $X$ ) is a QK space of quaternionic dimension $d=$ $h_{2,1}(Y)+1$ (resp. $d=h_{1,1}(X)+1$ ). It is obtained by the $c$-map construction from the vector multiplet moduli space $\mathcal{M}_{V}$ in type IIB (resp. IIA) theory compactified on the same Calabi-Yau manifold $Y$ (resp. $X$ ) [29,30]. $\mathcal{M}_{V}$ is a projective special Kähler manifold of dimension $2 d-2$, representing the moduli space of complex deformations of $Y$ (resp. complexified Kähler deformations of $X$ ), described in the standard way $[31,32]$ by a holomorphic prepotential $F\left(X^{\Lambda}\right)(\Lambda=0, \ldots, d-1)$, homogeneous of degree two.

As shown in $[33,34]$, the Lagrangian describing the Swann bundle of $\mathcal{M}$ is given by

$$
\mathcal{L}=\operatorname{Im}\left[\oint_{C_{+}} \frac{\mathrm{d} \zeta}{2 \pi \mathrm{i} \zeta} \frac{F\left(\eta^{\Lambda}\right)}{\eta^{b}}\right]
$$

where $\eta^{I}(I=b, 0, \ldots, d-1)$ are $\mathcal{O}(2)$ multiplets parameterized as in (3.4), and the contour $C_{+}$encloses the $\operatorname{root} \zeta_{+}$of $\zeta \eta^{b}(\zeta)$ (given in Eq. (3.34)) counter-clockwise. This 
can be cast in our general framework (3.23) by introducing four patches ${ }^{21}$ on $\mathbb{C} P^{1}$, centered at $0, \infty, \zeta_{+}, \zeta_{-}$, with transition functions

$$
H_{\text {tree }}^{[0+]}=-\frac{\mathrm{i}}{2} \frac{F\left(\eta^{\Lambda}\right)}{\eta^{\mathrm{b}}}, \quad H_{\text {tree }}^{[0-]}=-\frac{\mathrm{i}}{2} \frac{\bar{F}\left(\eta^{\Lambda}\right)}{\eta^{\mathrm{b}}}, \quad H_{\text {tree }}^{[0 \infty]}=0 .
$$

The contour integral (4.1) was evaluated in [17] (generalizing a previous computation in $[33,34]$ restricted to the locus $\left.v^{b}=\bar{v}^{b}=0\right)$, resulting in

$$
\mathcal{L}(v, \bar{v}, x)=\frac{1}{2 \mathrm{i} r^{b}}\left(F\left(\eta_{+}^{\Lambda}\right)-\bar{F}\left(\eta_{-}^{\Lambda}\right)\right) .
$$

The hyperkähler potential $\chi$ following by Legendre transform is given by [17]

$$
\chi=\frac{v^{b} \bar{v}^{b}}{\left(r^{b}\right)^{3}} K\left(\eta_{+}^{\Lambda}, \eta_{-}^{\Lambda}\right)
$$

where

$$
K(Z, \bar{Z}) \equiv \mathrm{i}\left(\bar{Z}^{\Lambda} F_{\Lambda}(Z)-Z^{\Lambda} \bar{F}_{\Lambda}(\bar{Z})\right) \equiv e^{-\mathcal{K}(Z, \bar{Z})}
$$

The hyperkähler potential $\chi$ may be further expressed in terms of the complex coordinates $v^{I}, w_{I}$ and their complex conjugate by means of the Hesse potential associated to the special Kähler manifold $\mathcal{M}_{V}[17]$.

The momentum coordinates $\mu_{I}^{[0]}$ for this geometry can be evaluated using (3.25) (away from the locus where the zeros of $\eta^{b}$ collide with other singularities of $F\left(\eta^{\Lambda}\right)$ ):

$$
\begin{aligned}
\mu_{\Lambda}^{[0]}= & \frac{\mathrm{i}}{2} \varrho_{\Lambda}-\frac{1}{4 \mathrm{i} r^{\mathrm{b}}}\left[\frac{\zeta+\zeta_{+}}{\zeta-\zeta_{+}} F_{\Lambda}\left(\eta_{+}\right)-\frac{\zeta+\zeta_{-}}{\zeta-\zeta_{-}} \bar{F}_{\Lambda}\left(\eta_{-}\right)\right], \\
\mu_{\mathrm{b}}^{[0]}= & \frac{\mathrm{i}}{2} \varrho_{b}+\frac{\zeta}{2 \mathrm{i}\left(r^{\mathrm{b}}\right)^{2}}\left[\frac{\zeta_{+} F\left(\eta_{+}\right)}{\left(\zeta-\zeta_{+}\right)^{2}}+\frac{\zeta-\bar{F}\left(\eta_{-}\right)}{\left(\zeta-\zeta_{-}\right)^{2}}\right]+\frac{x^{\mathrm{b}}}{4 \mathrm{i}\left(r^{\mathrm{b}}\right)^{3}}\left[\frac{\zeta+\zeta_{+}}{\zeta-\zeta_{+}} F\left(\eta_{+}\right)-\frac{\zeta+\zeta_{-}}{\zeta-\zeta_{-}} \bar{F}\left(\eta_{-}\right)\right] \\
& -\frac{1}{4 \mathrm{i}\left(r^{\mathrm{b}}\right)^{2}}\left[\frac{\zeta+\zeta_{+}}{\zeta-\zeta_{+}} F_{\Lambda}\left(\eta_{+}\right)\left(\frac{v^{\Lambda}}{\zeta_{+}}+\zeta_{+} \bar{v}^{\Lambda}\right)+\frac{\zeta+\zeta_{-}}{\zeta-\zeta_{-}} \bar{F}_{\Lambda}\left(\eta_{-}\right)\left(\frac{v^{\Lambda}}{\zeta-}+\zeta_{-} \bar{v}^{\Lambda}\right)\right] .
\end{aligned}
$$

Since $H^{[0 \infty]}=0$, the momentum coordinates around the south pole are given by $\mu_{I}^{[\infty]}=$ $\mu_{I}^{[0]}$, and one may check that the reality conditions (2.34) are indeed satisfied.

The multiplets $\mu_{\Lambda}^{[0]}$ and $\mu_{b}^{[0]}$ have a first order and second order pole at $\zeta=\zeta_{ \pm}$, respectively, while being regular elsewhere. It is readily checked that the combinations

$$
\mu_{\Lambda}^{[+]}=\mu_{\Lambda}^{[0]}-\frac{\mathrm{i}}{2} \frac{F_{\Lambda}(\eta)}{\eta^{b}}, \quad \mu_{b}^{[+]}=\mu_{b}^{[0]}+\frac{\mathrm{i}}{2} \frac{F(\eta)}{\left(\eta^{b}\right)^{2}},
$$

related to $\mu_{I}^{[0]}$ by the symplectomorphism generated by $H_{\text {tree }}^{[0+]}$, are regular at $\zeta=\zeta_{+}$, while being singular at $\zeta=\zeta_{-}$and other possible singularities of $F(\eta)$. Indeed, evaluating $\mu_{I}^{[+]}$at $\zeta=\zeta_{+}$yields

$$
\mu_{\Lambda}^{+}=\frac{\mathrm{i}}{2} \varrho_{\Lambda}-\frac{\mathrm{i} x^{b}}{4\left(r^{b}\right)^{2}}\left[F_{\Lambda}\left(\eta_{+}\right)-\bar{F}_{\Lambda}\left(\eta_{-}\right)\right]+\frac{\mathrm{i}}{2 r^{b}} F_{\Lambda \Sigma}\left(\eta_{+}\right)\left(\frac{v^{\Sigma}}{\zeta_{+}}+\bar{v}^{\Sigma} \zeta_{+}\right)
$$

21 Since $H^{[0 \infty]}=0, \mathcal{U}_{0}$ and $\mathcal{U}_{\infty}$ are really one and the same patch. We further assume that all singularities of $F(\eta)$ belong to either $\mathcal{U}_{0}$ or $\mathcal{U}_{\infty}$, but not to $\mathcal{U}_{ \pm}$. 
and

$$
\begin{aligned}
\mu_{b}^{+}= & \frac{\mathrm{i}}{2} \varrho_{b}+\mathrm{i} \frac{\left(x^{b}\right)^{2}-2 v^{b} \bar{v}^{b}}{4\left(r^{b}\right)^{4}}\left[F\left(\eta_{+}\right)-\bar{F}\left(\eta_{-}\right)\right] \\
& -\frac{\mathrm{i} x^{b}}{4\left(r^{b}\right)^{3}}\left[\left(\frac{v^{\Lambda}}{\zeta_{+}}+\zeta_{+} \bar{v}^{\Lambda}\right) F_{\Lambda}\left(\eta_{+}\right)+\left(\frac{v^{\Lambda}}{\zeta_{-}}+\zeta_{-} \bar{v}^{\Lambda}\right) \bar{F}_{\Lambda}\left(\eta_{-}\right)\right] \\
& -\frac{\mathrm{i}\left(\bar{v}^{b} v^{\Lambda}-v^{b} \bar{v}^{\Lambda}\right)}{2\left(r^{b}\right)^{3}} F_{\Lambda}\left(\eta_{+}\right)+\frac{\mathrm{i}}{4\left(r^{b}\right)^{2}} F_{\Lambda \Sigma}\left(\frac{v^{\Sigma}}{\zeta_{+}}+\zeta_{+} \bar{v}^{\Sigma}\right)\left(\frac{v^{\Lambda}}{\zeta_{+}}+\zeta_{+} \bar{v}^{\Lambda}\right) .
\end{aligned}
$$

Similarly, the combinations

$$
\mu_{\Lambda}^{[-]}=\mu_{\Lambda}^{[0]}-\frac{\mathrm{i}}{2} \frac{\bar{F}_{\Lambda}(\eta)}{\eta^{\mathrm{b}}}, \quad \mu_{\mathrm{b}}^{[-]}=\mu_{\mathrm{b}}^{[0]}+\frac{\mathrm{i}}{2} \frac{\bar{F}(\eta)}{\left(\eta^{\mathrm{b}}\right)^{2}}
$$

related to $\mu_{I}^{[0]}$ by the symplectomorphism generated by $H^{[0-]}$, are regular at $\zeta=\zeta_{-}$, while being singular at $\zeta=\zeta_{+}$and other possible singularities of $F(\eta)$.

We note that the multiplets $\mu_{I}$ may be obtained independently by making use of the special symmetry properties of the QK metrics in the image of the $c$-map, namely the existence of an extended Heisenberg group of tri-holomorphic isometries [29,30]. Upon lifting them to the Swann bundle, these isometries are generated by the holomorphic Killing vector fields [17]

$$
\begin{aligned}
& K=-\frac{\mathrm{i}}{4} \partial_{w_{b}}, \quad P^{\Lambda}=\frac{\mathrm{i}}{2} \partial_{w_{\Lambda}}, \quad Q_{\Lambda}=w_{\Lambda} \partial_{w^{b}}-v^{\mathrm{b}} \partial_{v^{\Lambda}}, \\
& M=w_{b} \partial_{w^{b}}-v^{b} \partial_{v^{b}}+\frac{1}{2} w_{\Lambda} \partial_{w_{\Lambda}}-\frac{1}{2} v^{\Lambda} \partial_{v^{\Lambda}} .
\end{aligned}
$$

The commuting isometries $K, P^{\Lambda}$ are manifest in the $\mathcal{O}(2)$ projective superfield construction; their $\mathcal{O}(2)$-valued moment maps are just the $\mathcal{O}(2)$ multiplets $\eta^{b}, \eta^{\Lambda}$. The moment maps, $\lambda_{\Lambda}, \lambda_{b}$ associated to the remaining isometries $Q_{\Lambda}$ and $M$ provide $d+1$ additional global $\mathcal{O}(2)$ sections

$$
\begin{aligned}
\lambda_{\Lambda}= & v^{b} w_{\Lambda} / \zeta+\left(w_{\Lambda} \partial_{w^{b}} \chi-v^{b} \partial_{v^{\Lambda}} \chi\right)+\bar{v}^{b} \bar{w}_{\Lambda} \zeta \\
\lambda_{b}= & \left(v^{b} w_{b}+\frac{1}{2} v^{\Lambda} w_{\Lambda}\right) \zeta^{-1}+\left(w_{b} \partial_{w^{b}} \chi-v^{b} \partial_{v^{b}} \chi+\frac{1}{2} w_{\Lambda} \partial_{w_{\Lambda}} \chi-\frac{1}{2} v^{\Lambda} \partial_{v^{\Lambda}} \chi\right) \\
& +\left(\bar{v}^{b} \bar{w}_{\Lambda}+\frac{1}{2} \bar{v}^{\Lambda} \bar{w}_{\Lambda}\right) \zeta .
\end{aligned}
$$

Matching the leading terms in the expansion around $\zeta=0$, one readily checks that the momentum coordinates around the north pole are given in terms of the global $\mathcal{O}(2)$ sections

$$
\mu_{\Lambda}^{[0]}=\frac{\lambda_{\Lambda}}{\eta^{b}}, \quad \mu_{b}^{[0]}=\frac{\lambda_{b}}{\eta^{b}}-\frac{\lambda_{\Lambda} \eta^{\Lambda}}{2\left(\eta^{b}\right)^{2}} .
$$


4.2. One-loop correction. In type II theories compactified on a Calabi-Yau $Y$, the metric on the hypermultiplet moduli space receives a one-loop correction, proportional to the Euler number of $Y$ [35]. There is evidence that there are no perturbative corrections to the hypermultiplet metric beyond one-loop [36]. ${ }^{22}$ As shown in [36], the one-loop correction can be described in the projective superspace formalism by adding a term

$$
\mathcal{L}_{1-\text { loop }}=2 c \oint_{\mathcal{C}} \frac{\mathrm{d} \zeta}{2 \pi \mathrm{i} \zeta} \eta^{\mathrm{b}} \log \eta^{\mathrm{b}}=-4 c\left(r^{\mathrm{b}}-x^{\mathrm{b}} \log \frac{x^{\mathrm{b}}+r^{\mathrm{b}}}{2\left|v^{\mathrm{b}}\right|}\right)
$$

to the Lagrangian (4.1), where $c$ is a constant determined in [36], proportional to the Euler character of the Calabi-Yau threefold. Here the contour $\mathcal{C}$ is a figure-eight contour around $\zeta_{+}$and $\zeta_{-}$, and the branch cuts in $\log \eta^{b}$ are chosen to extend from $\zeta_{+}$to 0 and $\zeta_{-}$to $\infty$ (see Sect. 3.4 in [1] for a more detailed discussion). Equivalently, the one-loop correction gives rise to additional contributions to the transition functions (4.2).

$$
H_{1-\text { loop }}^{[0+]}=2 c \eta^{b} \log \eta^{b}, \quad H_{1-\text { loop }}^{[0-]}=-2 c \eta^{b} \log \eta^{b}, \quad H_{1-\text { loop }}^{[0 \infty]}=0 .
$$

In particular, the transition functions are no longer homogeneous, but fall in the "quasihomogeneous" class, with anomalous dimensions

$$
c_{b}^{[0]}=c_{b}^{[\infty]}=0, \quad c_{b}^{[ \pm]}=\mp 2 c, \quad c_{\Lambda}^{[i]}=0 .
$$

The one-loop contribution to the hyperkähler potential is given by a simple correction to the formula (4.4) [28]

$$
\chi=\frac{v^{b} \bar{v}^{b}}{\left(r^{b}\right)^{3}} K\left(\eta_{+}, \eta_{-}\right)-4 c r^{b}
$$

in agreement with the general result (3.33).

Let us now determine the twistor lines for the one-loop corrected hypermultiplet moduli space. Starting from the general expression (3.25), the additional contribution (4.15) to the transition functions gives rise to extra terms in $\mu_{b ; T}^{[i]}$,

$$
\begin{aligned}
& \mu_{T ; b}^{[0]}=\mu_{b}^{[0] \text { tree }}+2 c \log \left(\left|\zeta_{+}\right| \frac{\zeta-\zeta_{-}}{\zeta-\zeta_{+}}\right), \\
& \mu_{T ; b}^{[+]}=\mu_{b}^{[+] \text {tree }}+2 c \log \left(-\frac{\left|v^{b}\right|\left(\zeta-\zeta_{-}\right)^{2}}{\zeta \zeta_{-}}\right)+2 c,
\end{aligned}
$$

while the other momentum coordinates remain unaltered, $\mu_{\Lambda}^{[i]}=\mu_{\Lambda}^{[i] \text { tree }}$. It is easy to check that the multiplet (4.18) transforms under $S U(2)$ transformations according to (A.6).

\footnotetext{
22 In the case of the universal hypermultiplet, this was established rigorously in [37]. See the end of Sect. 4.3 for a strengthening of the non-renormalization argument in [36].
} 
4.3. Superconformal quotient. The superconformal quotient of the HKC defined by (4.1) was studied in $[17,28] .{ }^{23}$ The dilation and $S U(2)$ invariant coordinates used in these references were given by

$$
\begin{aligned}
e^{2 U} & =\frac{\chi}{4 r^{b}}, \quad \zeta^{\Lambda}=\frac{\vec{r}^{b} \cdot \vec{r}^{\Lambda}}{\left(r^{b}\right)^{2}}, \quad z^{a}=\frac{\eta_{+}^{a}}{\eta_{+}^{0}}, \quad \tilde{\zeta}_{\Lambda}=-\mathrm{i}\left(w_{\Lambda}-\bar{w}_{\Lambda}\right)+\frac{x^{b}}{\left(r^{b}\right)^{2}} \operatorname{Re}\left[F_{\Lambda}\left(\eta_{+}\right)\right] \\
\sigma & =2 \mathrm{i}\left(w_{b}-\bar{w}_{b}\right)+\mathrm{i}\left(\frac{v^{\Lambda}}{v^{b}} w_{\Lambda}-\frac{\bar{v}^{\Lambda}}{\bar{v}^{b}} \bar{w}_{\Lambda}\right)-\frac{x^{b}}{\left(r^{b}\right)^{2}} \operatorname{Re}\left[\eta_{+}^{\Lambda} \tilde{\zeta}_{\Lambda}-F_{\Lambda}\left(\eta_{+}\right) \zeta^{\Lambda}\right]-4 \mathrm{i} c \log \frac{\eta_{+}^{0}}{\eta_{-}^{0}} .
\end{aligned}
$$

While the $S U(2)$-invariance of $\tilde{\zeta}_{\Lambda}$ and $\sigma$ directly is rather tedious to check, it can be made manifest by casting the resulting expressions in the form of a contour integral of an $\mathcal{O}(-2)$ section, e.g. when $c=0$,

$$
\tilde{\zeta}_{\Lambda}=-2 \mathrm{i} r^{b} \oint_{C^{+}} \frac{\mathrm{d} \zeta}{2 \pi \mathrm{i} \zeta} \frac{\mu_{\Lambda}^{[0]}}{\eta^{b}}, \quad \sigma=4 \mathrm{i} r^{b} \oint_{C^{+}} \frac{\mathrm{d} \zeta}{2 \pi \mathrm{i} \zeta}\left(\frac{\mu_{b}^{[0]}}{\eta^{b}}+\frac{\eta^{\Lambda} \mu_{\Lambda}^{[0]}}{2\left(\eta^{b}\right)^{2}}\right) .
$$

In the presence of the one-loop correction, these expressions may be generalized by performing the same replacement as in (3.39) and taking the real part.

We now relate the result (4.19) to the general $S U(2)$ and dilation invariant coordinates introduced in Sect. 3.2. Clearly, $U=\phi / 2, z^{a}=Z^{a}, \zeta^{\Lambda}=A^{\Lambda}$. On the other hand, evaluating (3.39) with the help of (4.8), (4.9) and (4.18) leads to

$$
\begin{aligned}
B_{\Lambda} & =\varrho_{\Lambda}+\frac{x^{b}}{\left(r^{b}\right)^{2}} \operatorname{Re} F_{\Lambda}\left(\eta_{+}\right)-A^{\Sigma} \operatorname{Re} F_{\Lambda \Sigma}\left(\eta_{+}\right), \\
B_{b} & =\varrho_{b}+\frac{1}{\left(r^{b}\right)^{2}} \operatorname{Re} F\left(\eta_{+}\right)-\frac{x^{\Lambda}+x^{b} A^{\Lambda}}{2\left(r^{b}\right)^{2}} \operatorname{Re} F_{\Lambda}\left(\eta_{+}\right)+\frac{1}{2} A^{\Lambda} A^{\Sigma} \operatorname{Re} F_{\Lambda \Sigma}\left(\eta_{+}\right)+2 \mathrm{i} c \log \frac{\eta_{+}^{0}}{\eta_{-}^{0}} .
\end{aligned}
$$

Thus the coordinates $B_{I}$ differ from $\sigma, \tilde{\zeta}_{\Lambda}$ by $S U(2)$ invariant terms,

$$
B_{\Lambda}=\tilde{\zeta}_{\Lambda}-A^{\Sigma} \operatorname{Re} F_{\Lambda \Sigma}(Z), \quad B_{b}=-\frac{1}{2} \sigma-\frac{1}{2} A^{\Lambda} B_{\Lambda} .
$$

The contact twistor lines can be found using the general formulae (3.50), (3.53),

$$
\begin{aligned}
\xi^{\Lambda}= & A^{\Lambda}+\mathcal{R}\left(\mathbf{z}^{-1} Z^{\Lambda}-\mathbf{z} \bar{Z}^{\Lambda}\right), \\
\tilde{\xi}_{\Lambda}^{[0]}= & \frac{\mathrm{i}}{2}\left[B_{\Lambda}+A^{\Sigma} \operatorname{Re} F_{\Lambda \Sigma}(Z)+\mathcal{R}\left(\mathbf{z}^{-1} F_{\Lambda}(Z)-\mathbf{z} \bar{F}_{\Lambda}(\bar{Z})\right)\right], \\
\tilde{\xi}_{b}^{[0]}=\frac{\mathrm{i}}{2} & {\left[B_{b}-\frac{1}{2} A^{\Lambda} A^{\Sigma} \operatorname{Re} F_{\Lambda \Sigma}(Z)+\mathcal{R}^{2} \operatorname{Re}\left(\bar{Z}^{\Lambda} F_{\Lambda}(Z)\right)\right.} \\
& \left.\quad-\mathcal{R} A^{\Lambda}\left(\mathbf{z}^{-1} F_{\Lambda}(Z)-\mathbf{z} \bar{F}_{\Lambda}(\bar{Z})\right)-\mathcal{R}^{2}\left(\mathbf{z}^{-2} F(Z)+\mathbf{z}^{2} \bar{F}(\bar{Z})\right)\right]-2 c \log \mathbf{z} .
\end{aligned}
$$

Finally, it remains to express $\mathcal{R}$, defined in (3.40) above, in terms of the base coordinates (3.36)-(3.39). For this purpose, one may substitute the one-loop corrected hyperkähler

23 Ref. [28] used a different contour prescription related to the one used here by a local gauge transformation, as we explain in Appendix B. As a result, the expressions for $\tilde{\zeta}_{\Lambda}$ and $\sigma$ acquired some additional terms. 
potential (4.17) into the definition of $\phi$, Eq. (3.38), and use the homogeneity property of $K(\cdot, \cdot)$ to obtain

$$
\mathcal{R}=2 e^{\frac{1}{2} \mathcal{K}(Z, \bar{Z})} \sqrt{e^{\phi}+c} .
$$

Introducing

$$
W \equiv F_{\Lambda}(Z) \zeta^{\Lambda}-Z^{\Lambda} \tilde{\zeta}_{\Lambda}
$$

and using (4.22), one may obtain the contact twistor lines for the one-loop corrected hypermultiplet geometry in the form found in [17],

$$
\begin{aligned}
\xi^{\Lambda} & =\zeta^{\Lambda}+\mathcal{R}\left(\mathbf{z}^{-1} Z^{\Lambda}-\mathbf{z} \bar{Z}^{\Lambda}\right), \\
-2 \mathrm{i} \tilde{\xi}_{\Lambda}^{[0]} & =\tilde{\zeta}_{\Lambda}+\mathcal{R}\left(\mathbf{z}^{-1} F_{\Lambda}-\mathbf{z} \bar{F}_{\Lambda}\right), \\
4 \mathrm{i} \tilde{\xi}_{b}^{[0]}+2 \mathrm{i} \tilde{\xi}_{\Lambda}^{[0]} \xi^{\Lambda} & =\sigma+\mathcal{R}\left(\mathbf{z}^{-1} W-\mathbf{z} \bar{W}\right)-8 \mathrm{i} c \log \mathbf{z} .
\end{aligned}
$$

We proceed to extract the one-loop corrected hypermultiplet metric from the twistor data on $\mathcal{Z}_{\mathcal{M}}$. Following the procedure outlined at the end of Sect. 2.5, we first compute the Laurent coefficients of $\tilde{\xi}_{I}^{[+]}$entering the $S U(2)$ connection (2.83),

$$
\begin{aligned}
& \tilde{\xi}_{\Lambda, 0}^{[+]}=\frac{\mathrm{i}}{2}\left(\tilde{\zeta}_{\Lambda}-F_{\Lambda \Sigma} \zeta^{\Sigma}\right), \quad \tilde{\xi}_{b, 0}^{[+]}=-\frac{\mathrm{i}}{4}\left(\sigma+\zeta^{\Lambda} \tilde{\zeta}_{\Lambda}-F_{\Lambda \Sigma} \zeta^{\Lambda} \zeta^{\Sigma}\right)-e^{\phi}+c, \\
& \tilde{\xi}_{\Lambda, 1}^{[+]}=\frac{1}{2} \mathcal{R} N_{\Lambda \Sigma} \bar{Z}^{\Sigma}-\frac{\mathrm{i}}{4 \mathcal{R}} F_{\Lambda \Sigma \Xi} \zeta^{\Sigma} \zeta^{\Xi} \\
& \tilde{\xi}_{b, 1}^{[+]}=-\frac{1}{2} \mathcal{R} N_{\Lambda \Sigma} \zeta^{\Lambda} \bar{Z}^{\Sigma}+\frac{\mathrm{i}}{12 \mathcal{R}} F_{\Lambda \Sigma \Xi} \zeta^{\Lambda} \zeta^{\Sigma} \zeta^{\Xi} .
\end{aligned}
$$

where $N_{\Lambda \Sigma} \equiv \mathrm{i}\left(F_{\Lambda \Sigma}-\bar{F}_{\Lambda \Sigma}\right)$, leading to the $S U$ (2) connection

$$
\begin{aligned}
& p_{+}=\frac{\mathrm{i}}{4} e^{-\phi} \mathcal{R} Z^{\Lambda}\left(\mathrm{d} \tilde{\zeta}_{\Lambda}-F_{\Lambda \Sigma} \mathrm{d} \zeta^{\Sigma}\right)=\left(p_{-}\right)^{*} \\
& p_{3}=\frac{1}{8} e^{-\phi}\left(\mathrm{d} \sigma+\tilde{\zeta}_{\Lambda} \mathrm{d} \zeta^{\Lambda}-\zeta^{\Lambda} \mathrm{d} \tilde{\zeta}_{\Lambda}+4\left(\mathrm{e}^{\phi}+c\right) \mathcal{A}_{K}\right),
\end{aligned}
$$

where $\mathcal{A}_{K} \equiv \mathrm{i}\left(\mathcal{K}_{a} \mathrm{~d} Z^{a}-\mathcal{K}_{\bar{a}} \mathrm{~d} \bar{Z}^{\bar{a}}\right)$ is the Kähler connection of the projective special Kähler base $\mathcal{M}_{V}$. A direct computation of the $(1,0)$ forms $(2.88)$ then yields

$$
\begin{aligned}
\Pi^{a}= & \mathcal{R}^{2} \mathrm{~d} Z^{a} \\
\tilde{\Pi}_{\Lambda}= & \frac{\mathrm{i}}{2} \mathcal{R}\left(\mathrm{d} \tilde{\zeta}_{\Lambda}-F_{\Lambda \Sigma} \mathrm{d} \zeta^{\Sigma}-F_{\Lambda \Sigma \Xi} \zeta^{\Sigma} \mathrm{d} Z^{\Xi}\right) \\
& +\frac{\mathrm{i}}{4 r} \mathcal{R}^{3}\left(\operatorname{Im}\left(F_{\Lambda \Sigma}\right) \bar{Z}^{\Sigma}+\frac{\mathrm{i}}{4 \mathcal{R}^{2}} F_{\Lambda \Sigma \Xi} \zeta^{\Lambda} \zeta^{\Xi}\right)\left(Z^{\mathrm{T}} \mathrm{d} \tilde{\zeta}_{\mathrm{T}}-F_{\mathrm{T}} \mathrm{d} \zeta^{\mathrm{T}}\right), \\
\tilde{\Pi}_{b}=- & \mathcal{R}\left[\frac{r+2 c}{r+c} \mathrm{~d} r+c \mathrm{~d} \mathcal{K}\right. \\
& +\frac{\mathrm{i}}{4}\left(\mathrm{~d} \sigma+\tilde{\zeta}_{\Lambda} \mathrm{d} \zeta^{\Lambda}+\zeta^{\Lambda} \mathrm{d} \tilde{\zeta}_{\Lambda}-F_{\Lambda \Sigma \Xi} \zeta^{\Lambda} \zeta^{\Sigma} \mathrm{d} Z^{\Xi}-2 F_{\Lambda \Sigma} \zeta^{\Lambda} \mathrm{d} \zeta^{\Sigma}\right) \\
& \left.+\frac{\mathrm{i}}{4 r}\left(\mathcal{R}^{2} \operatorname{Im}\left(F_{\Lambda \Sigma}\right) \zeta^{\Lambda} \bar{Z}^{\Sigma}+\frac{\mathrm{i}}{12} F_{\Lambda \Sigma \Xi} \zeta^{\Lambda} \zeta^{\Sigma} \zeta^{\Xi}\right)\left(Z^{\mathrm{T}} \mathrm{d} \tilde{\zeta}_{\mathrm{T}}-F_{\mathrm{T}} \mathrm{d} \zeta^{\mathrm{T}}\right)\right]
\end{aligned}
$$


where $r \equiv e^{\phi}$. Taking linear combinations, a basis of $(1,0)$ forms can be chosen as

$$
\begin{aligned}
& \mathrm{d} Z^{a}, \quad f_{a}^{\Lambda}\left(\mathrm{d} \tilde{\zeta}_{\Lambda}-F_{\Lambda \Sigma} \mathrm{d} \zeta^{\Sigma}\right) \\
& Z^{\Lambda} \mathrm{d} \tilde{\zeta}_{\Lambda}-F_{\Lambda} \mathrm{d} \zeta^{\Lambda}, \quad \frac{r+2 c}{r+c} \mathrm{~d} r+\frac{\mathrm{i}}{4}\left(\mathrm{~d} \sigma+\tilde{\zeta}_{\Lambda} \mathrm{d} \zeta^{\Lambda}-\zeta^{\Lambda} \mathrm{d} \tilde{\zeta}_{\Lambda}\right)+c \mathrm{~d} \mathcal{K}
\end{aligned}
$$

where $f_{a}^{\Lambda}=e^{\mathcal{K} / 2}\left(\partial_{a} Z^{\Lambda}+\partial_{a} \mathcal{K} Z^{\Lambda}\right)$, generalizing the one-forms $e^{a}, E_{a}, u, v$ introduced in [30] for $c \neq 0$. Finally, computing the Kähler form (2.85) in this basis and raising the indices, one obtains the one-loop corrected metric on the hypermultiplet moduli space $[28,36]$,

$$
\begin{aligned}
d s^{2}= & \frac{r+2 c}{r^{2}(r+c)} \mathrm{d} r^{2}-\frac{1}{r}\left(N^{\Lambda \Sigma}-\frac{2(r+c)}{r K} Z^{\Lambda} \bar{Z}^{\Sigma}\right)\left(\mathrm{d} \tilde{\zeta}_{\Lambda}-F_{\Lambda \Theta} \mathrm{d} \zeta^{\Theta}\right) \\
& \times\left(\mathrm{d} \tilde{\zeta}_{\Sigma}-\bar{F}_{\Sigma \Xi} \mathrm{d} \zeta^{\Xi}\right) \\
& +\frac{r+c}{16 r^{2}(r+2 c)}\left(\mathrm{d} \sigma+\tilde{\zeta}_{\Lambda} d \zeta^{\Lambda}-\zeta^{\Lambda} \mathrm{d} \tilde{\zeta}_{\Lambda}+4 c \mathcal{A}_{K}\right)^{2}+\frac{4(r+c)}{r} \mathcal{K}_{a \bar{b}} \mathrm{~d} Z^{a} \mathrm{~d} \bar{Z}^{\bar{b}}
\end{aligned}
$$

which identifies $\phi$ as the four-dimensional dilaton.

It is worthwhile noting that the one-loop correction changes the topology of the fibration of the $\sigma$-circle over the torus coordinatized by $\zeta^{\Lambda}, \tilde{\zeta}_{\Lambda}$, by a term proportional to $\mathcal{A}_{K}$ [38]. Any perturbative correction to the hypermultiplet metric beyond one-loop would presumably induce extra terms in the connection on the $\sigma$ circle bundle proportional to a positive power of $r$, and would therefore conflict with the quantization of its first Chern class. This observation reinforces the arguments given in [36] ruling out perturbative corrections to the hypermultiplet metric beyond one-loop.

\section{Linear Deformations of $\mathcal{O}(2)$ Quaternionic-Kähler Spaces}

In this section, we study the infinitesimal deformations of $4 d$-dimensional QK spaces $\mathcal{M}$ with $d+1$ commuting isometries, which preserve the QK property but may break some or all of the isometries. Our strategy is to apply the general analysis of linear deformations of $\mathcal{O}(2)$ HK spaces developed in [1] to the Swann bundle $\mathcal{S}$ of $\mathcal{M}$, restricting to deformations which preserve the superconformal invariance property. As explained in the introduction, it is possible to bypass the Swann bundle and work directly with the twistor space $\mathcal{Z}_{\mathcal{M}}$. This strategy will be realized in Sect. 5.2.

5.1. Linear deformations of $\mathcal{O}(2)$ hyperkähler cones. As explained in [1], deformations of HK spaces $\mathcal{S}$ are conveniently described by perturbing the transition functions $S^{[i j]}$ which encode the holomorphic symplectic structure on the twistor space $\mathcal{Z}_{\mathcal{S}}$,

$$
S^{[i j]}\left(v_{[i]}, \mu^{[j]}, \zeta\right)=f_{i j}^{-2} v_{[i]}^{I} \mu_{I}^{[j]}-\tilde{H}^{[i j]}\left(v_{[i]}, \zeta\right)-\tilde{H}_{(1)}^{[i j]}\left(v_{[i]}, \mu^{[j]}, \zeta\right),
$$

and working out the perturbations $\hat{v}_{[i]}^{I}, \hat{\mu}_{I}^{[i]}$ of the twistor lines,

$$
v_{[i]}^{I}=\zeta f_{0 i}^{-2} \eta^{I}+\hat{v}_{[i]}^{I}, \quad \mu_{I}^{[i]}=\breve{\mu}_{I}^{[i]}+\hat{\mu}_{I}^{[i]}
$$


to first order in the perturbations $\tilde{H}_{(1)}^{[i j]}$. Here and below, unperturbed quantities are

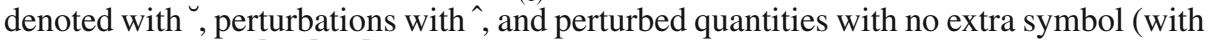
the exception of $\eta^{I}, v^{I}, x^{I}$ which will continue to denote unperturbed quantities).

As shown in Sect. 3.1, superconformal invariance restricts the undeformed transition functions $\tilde{H}^{[i j]}\left(v_{[i]}, \zeta\right)$ to be homogeneous of degree one in $v_{[i]}$, and without any explicit dependence on $\zeta$ except for some factors of $f_{i j}$. The same reasoning shows the perturbation $\tilde{H}_{(1)}^{[i j]}$ should satisfy the same conditions, namely

$$
\tilde{H}_{(1)}^{[i j]}\left(v_{[i]}, \mu^{[j]}, \zeta\right)=f_{i j}^{-2} \hat{H}_{(1)}^{[i j]}\left(v_{[i]}^{I}, \mu_{I}^{[j]}+c_{I}^{[j]} \log \left(f_{i j}^{-2} v_{[i]}^{b}\right)\right),
$$

where $\hat{H}_{(1)}^{[i j]}$ is a homogeneous function of degree one in its first argument. ${ }^{24}$ Following [1], we now trade $\tilde{H}_{(1)}^{[i j]}$ for

$$
\begin{aligned}
H_{(1)}^{[i j]}\left(\eta, \breve{\mu}^{[j]}, \zeta\right) & \equiv \zeta^{-1} f_{0 j}^{2} \tilde{H}_{(1)}^{[i j]}\left(\zeta f_{0 i}^{-2} \eta, \breve{\mu}^{[j]}, \zeta\right) \\
& =\hat{H}_{(1)}^{[i j]}\left(\eta^{I}, \breve{\mu}_{I}^{[j]}+c_{I}^{[j]} \log \left(f_{0 j}^{-2} \zeta \eta^{b}\right)\right) .
\end{aligned}
$$

We then perform the gauge transformation (3.13) to obtain

$$
H_{(1)}^{[i j]}\left(\eta, \breve{\mu}_{T}^{[j]}, \zeta\right)=\hat{H}_{(1)}^{[i j]}\left(\eta^{I}, \breve{\mu}_{T ; I}^{[j]}+c_{I}^{[j]} \log \eta+c_{I}^{[0]} \log \zeta\right) .
$$

Finally, we trade the argument $\breve{\mu}_{I ; T}^{[j]}$ for the real multiplet

$$
\rho_{I}(\zeta) \equiv-\mathrm{i}\left(\breve{\mu}_{T ; I}^{[0]}+\breve{\mu}_{T ; I}^{[\infty]}\right)-\mathrm{i} c_{I}^{[0 \infty]} \log \zeta .
$$

This quantity has the advantage of having non-anomalous $\mathcal{O}(0)$ transformations, moreover the reality conditions are also automatically satisfied provided $H_{(1)}^{[i \bar{i}]}$ is a real function of $\eta^{I}$ and $\rho_{I}$. After these redefinitions, $H_{(1)}^{[i j]}$ is now a function of $\eta^{I}, \rho_{I}$, homogeneous of degree one in $\eta^{I}$, and with no explicit dependence on $\zeta$. In addition, it must satisfy the co-cycle condition (3.8) and is subject to the gauge equivalence (3.9), where $G^{[i]}$ is now a function of $\eta^{I}, \rho_{I}$ regular in the patch $\hat{\mathcal{U}}_{i}$.

We may now borrow the results from [1], Sect. 5. In particular, the first order variation of the HK twistor lines is given by

$$
\begin{aligned}
\hat{v}_{[i]}^{I} & =\mathrm{i} f_{0 i}^{-2} \sum_{j} \oint_{C_{j}} \frac{\mathrm{d} \zeta^{\prime}}{2 \pi \mathrm{i} \zeta^{\prime}} \frac{\zeta^{3}+\zeta^{\prime 3}}{\zeta^{\prime}\left(\zeta^{\prime}-\zeta\right)} H_{(1)}^{[0 j] I}\left(\zeta^{\prime}\right), \\
\hat{\mu}_{T ; I}^{[i]} & =\sum_{j} \oint_{C_{j}} \frac{\mathrm{d} \zeta^{\prime}}{2 \pi \mathrm{i} \zeta^{\prime}} \frac{\zeta+\zeta^{\prime}}{2\left(\zeta^{\prime}-\zeta\right)} G_{I}^{[0 j]}\left(\zeta^{\prime}\right)
\end{aligned}
$$

with

$$
\begin{aligned}
& H_{I} \equiv \partial_{\eta^{I}} H, \quad H_{I J} \equiv \partial_{\eta^{I}} \partial_{\eta^{J}} H, \quad H_{(1) I} \equiv \partial_{\eta^{I}} H_{(1)}, \quad H_{(1)}^{I} \equiv \partial_{\rho_{I}} H_{(1)}, \\
& G_{I}^{[i j]} \equiv H_{(1)}^{[i j]}{ }_{I}+\mathrm{i} H_{(1)}^{[i j] J}\left(H_{I J}^{[j 0]}+H_{I J}^{[j \infty]}\right)+\zeta^{-1} f_{0 i}^{2} \hat{v}_{[i]}^{J} H_{I J}^{[i j]} \text {. }
\end{aligned}
$$

24 For simplicity, we do not consider deformations of the anomalous dimensions. However, it may be checked that all formulae below continue to hold provided $c_{I}^{[i]}$ denote the total perturbed anomalous dimensions. 
The corresponding deformations of the Kähler potential can be conveniently described by introducing the deformed Lagrangian

$$
\mathcal{L}(v, \bar{v}, x, \varrho)=\sum_{j} \oint_{C_{j}} \frac{\mathrm{d} \zeta}{2 \pi \mathrm{i} \zeta}\left(H^{[0 j]}(\eta)+H_{(1)}^{[0 j]}(\eta, \rho)\right) .
$$

So defined, it is a function of the complex variables $v^{I}$. However, after perturbations $v_{I}$ are no longer Darboux coordinates. Instead, a system of complex Darboux coordinates of the deformed $\mathrm{HKC}$, such that $\omega_{\mathcal{S}}^{+}=\mathrm{d} w_{I} \wedge \mathrm{d} u^{I}$, is given by

$$
u^{I}=v^{I}+\mathrm{i} \partial_{\varrho_{I}} \sum_{j} \oint_{C_{j}} \frac{\mathrm{d} \zeta}{2 \pi \mathrm{i}} H_{(1)}^{[0 j]}, \quad w_{I}=\frac{\mathrm{i}}{2} \varrho_{I}+\frac{1}{2} \partial_{x^{I}} \mathcal{L}(u, \bar{u}, x, \varrho),
$$

where in the second relation the arguments $v^{I}$ of the Lagrangian are replaced by the new complex variables $u^{I}$ and the derivative is evaluated keeping $u^{I}, \bar{u}^{I}$ and $\varrho_{I}$ fixed. Similarly, the Kähler potential for the deformed HK metric is given by the Legendre transform of the deformed Lagrangian (5.10) but written as a function of the new variables

$$
\chi(u, \bar{u}, w, \bar{w})=\left\langle\mathcal{L}(u, \bar{u}, x, \varrho)-x^{I}\left(w_{I}+\bar{w}_{I}\right)\right\rangle_{x^{I}} .
$$

In particular, the variation of the hyperkähler potential is given by a Penrose-type integral,

$$
\chi_{(1)}(u, \bar{u}, w, \bar{w})=\sum_{j} \oint_{C_{j}} \frac{\mathrm{d} \zeta}{2 \pi \mathrm{i} \zeta} H_{(1)}^{[0 j]}(\eta, \rho)
$$

We now verify that the perturbed HK manifold is indeed a HKC (as is of course guaranteed by construction). Using the quasi-homogeneity property of $H_{(1)}(\eta, \rho)$, and in particular the property

$$
\left(u^{I} \partial_{u^{I}}-\bar{u}^{I} \partial_{\bar{u} I}+\zeta \partial_{\zeta}\right) \rho_{J}=0,
$$

it is easily checked that $\mathcal{L}$ satisfies

$$
\begin{aligned}
u^{I} \mathcal{L}_{u^{I}}-\bar{u}^{I} \mathcal{L}_{\bar{u}^{I}} & =-2 \mathrm{i} c_{I}^{[0]} \mathcal{L}_{\varrho_{I}}, \\
x^{I} \mathcal{L}_{x^{I}}+u^{I} \mathcal{L}_{u^{I}}+\bar{u}^{I} \mathcal{L}_{\bar{u}^{I}} & =\mathcal{L}-2 c_{I}^{[0]} x^{I} .
\end{aligned}
$$

Together with the identities

$$
\begin{aligned}
& \left(\partial_{x^{I}}+\mathrm{i} \mathcal{L}_{x^{I} x^{K}} \partial_{\varrho_{K}}\right)\left(\partial_{x^{J}}-\mathrm{i} \mathcal{L}_{x^{J} x^{L}} \partial_{\varrho_{L}}\right) \mathcal{L} \\
& \quad+\left(\partial_{u^{I}}-\mathrm{i} \mathcal{L}_{u^{I} x^{K}} \partial_{\varrho_{K}}\right)\left(\partial_{\bar{u}^{J}}+\mathrm{i} \mathcal{L}_{\bar{u}^{J} x^{L}} \partial_{\varrho_{L}}\right) \mathcal{L}=0, \\
& \left(\partial_{x^{I}}+\mathrm{i} \mathcal{L}_{x^{I} x^{K}} \partial_{\varrho_{K}}\right)\left(\partial_{u^{J}}-\mathrm{i} \mathcal{L}_{u^{J} x^{L}} \partial_{\varrho_{L}}\right) \mathcal{L} \\
& \quad-\left(\partial_{x^{J}}+\mathrm{i} \mathcal{L}_{x^{J} x^{K}} \partial_{\varrho_{K}}\right)\left(\partial_{u^{I}}-\mathrm{i} \mathcal{L}_{u^{I} x^{L}} \partial_{\varrho_{L}}\right) \mathcal{L}=0,
\end{aligned}
$$


these equations guarantee that $\mathcal{L}$ satisfies the constraints of superconformal invariance. Moreover, from (2.17), one may compute the homothetic Killing vector and the Killing vectors for the $S U(2)$ isometric action,

$$
\begin{aligned}
& \chi^{u^{I}}=2 u^{I}, \quad \chi^{w_{I}}=-2 c_{I}^{[0]} . \\
& \delta u^{I}=\mathrm{i} \epsilon_{3} u^{I}+\epsilon_{+}\left(x^{I}+\mathrm{i} \mathcal{L}_{\varrho_{I}}\right), \quad \delta \bar{u}^{I}=-\mathrm{i} \epsilon_{3} \bar{v}^{I}+\epsilon_{-}\left(x^{I}-\mathrm{i} \mathcal{L}_{\varrho_{I}}\right), \\
& \delta w_{I}=\epsilon_{+} \mathcal{L}_{u^{I}}-\mathrm{i} \epsilon_{3} c_{I}^{[0]}, \quad \delta \bar{w}_{I}=\epsilon_{-} \mathcal{L}_{\bar{u}^{I}}+\mathrm{i} \epsilon_{3} c_{I}^{[0]} .
\end{aligned}
$$

In particular, the homothetic Killing vector is holomorphic and identical to the undeformed case. Moreover, one may check that the one-form obtained by lowering the index on $k^{+}$using the deformed metric reproduces the Liouville form (2.64) on $\mathcal{S}$ in the patch $i=0[4]$.

5.2. Perturbed contact twistor lines. In order to extract the deformed quaternionicKähler metric on $\mathcal{M}$, one possible strategy is to study the deformations of the superconformal quotient: this computationally intensive approach is outlined in Appendix C. However, it turns out to be more economic and elegant to work directly with the complex contact structure on the twistor space $\mathcal{Z}_{\mathcal{M}}$, without reference to the Swann bundle and its twistor space.

For this purpose, let us recast the deformed symplectomorphism (5.1) into the form of the contact transformations (2.71). Introducing the same coordinates as in (2.65), it is easy to check that the deformed contact transformations are generated by the following transition functions:

$$
\hat{S}^{[i j]}\left(\xi_{[i]}^{\Lambda}, \tilde{\xi}_{I}^{[j]}\right)=\tilde{\xi}_{b}^{[j]}+\xi_{[i]}^{\Lambda} \tilde{\xi}_{\Lambda}^{[j]}-\hat{H}^{[i j]}\left(\xi_{[i]}^{\Lambda}\right)-\hat{H}_{(1)}^{[i j]}\left(\xi_{[i]}^{\Lambda}, \tilde{\xi}_{I}^{[j]}\right),
$$

where $\tilde{\xi}_{I}^{[j]}$ should be replaced by $\tilde{\xi}_{I}^{[j]}+c_{I}^{[j]} \log \left(\hat{f}_{i j}^{-2}\right)$. However, it follows from (2.73) that

$$
\hat{f}_{i j}^{2} \approx 1-\partial_{\tilde{\xi}_{b}^{[j]}} \hat{H}_{(1)}^{[i j]}
$$

so that its logarithm is of first order in the perturbation already. Therefore, to the first order it is consistent to neglect the term $c_{I}^{[j]} \log \left(\hat{f}_{i j}^{-2}\right)$ in the argument of $\hat{H}_{(1)}^{[i j]}$, and take $\hat{H}_{(1)}^{[i j]}$ to be an arbitrary function of the undeformed coordinates $\breve{\xi}_{[i]}^{\Lambda}$ and $\breve{\tilde{\xi}}_{I}^{[j]}$. As a result, one finds the following deformed contact transformations:

$$
\xi_{[i]}^{\Lambda}=\xi_{[j]}^{\Lambda}-T_{[i j]}^{\Lambda}, \quad \tilde{\xi}_{I}^{[i]}=\tilde{\xi}_{I}^{[j]}-\tilde{T}_{I}^{[i j]},
$$

where, in view of later applications, we abbreviated

$$
\begin{aligned}
T_{[i j]}^{\Lambda} & \equiv-\partial_{\tilde{\xi}_{\Lambda}^{[j]}} \hat{H}_{(1)}^{[i j]}+\xi_{[i]}^{\Lambda} \partial_{\tilde{\xi}_{b}^{[j]}} \hat{H}_{(1)}^{[i j]} \\
\tilde{T}_{\Lambda}^{[i j]} & \equiv \partial_{\xi_{[i]}^{\Lambda}}\left(\hat{H}^{[i j]}+\hat{H}_{(1)}^{[i j]}\right)-c_{\Lambda}^{[j]} \partial_{\tilde{\xi}_{b}^{[j]}} \hat{H}_{(1)}^{[i j]} \\
\tilde{T}_{b}^{[i j]} & \equiv\left(\hat{H}^{[i j]}+\hat{H}_{(1)}^{[i j]}\right)-\xi_{[i]}^{\Lambda} \partial_{\xi_{[i]}^{\Lambda}}\left(\hat{H}^{[i j]}+\hat{H}_{(1)}^{[i j]}\right)+c_{I}^{[i j]} \xi_{[i]}^{I}+c_{\Lambda}^{[j]} \partial_{\tilde{\xi}_{\Lambda}^{[j]}} \hat{H}_{(1)}^{[i j]}
\end{aligned}
$$

As usual, the functions $\hat{H}_{(1)}^{[i j]}$ must satisfy the co-cycle condition (3.8) and are defined up to the gauge equivalence (3.10). Thus, they define an element in the Čech cohomology 
$\operatorname{group}^{25} H^{1}\left(\mathcal{Z}_{\mathcal{M}}, \mathcal{O}(2)\right)$, realizing Lebrun's assertion that this group classifies the QK deformations of $\mathcal{M}$ [14].

We now determine the deformed contact twistor lines. For definiteness we focus on the coordinate $\xi_{[+]}^{\Lambda}\left(\mathbf{z}, x^{\mu}\right)$ around $\zeta=\zeta_{+}$, i.e. $\mathbf{z}=0$. The pole and the constant term in the Laurent expansion (2.80) are readily obtained by contour integrating around $\mathbf{z}=0$ :

$$
\xi_{[+]}^{\Lambda}\left(\mathbf{z}, x^{\mu}\right)=Y_{+}^{\Lambda} \mathbf{z}^{-1}+A_{+}^{\Lambda}+\mathcal{O}(\mathbf{z}),
$$

where

$$
A_{+}^{\Lambda}=\oint_{0} \frac{\mathrm{d} \mathbf{z}}{2 \pi \mathrm{i} \mathbf{z}} \xi_{[+]}^{\Lambda}, \quad Y_{+}^{\Lambda}=\oint_{0} \frac{\mathrm{d} \mathbf{z}}{2 \pi \mathrm{i}} \xi_{[+]}^{\Lambda} .
$$

On the other hand, the full Laurent series expansion of $\xi_{[+]}^{\Lambda}\left(\mathbf{z}, x^{\mu}\right)$ at $\mathbf{z}=0$ is given by the series

$$
\xi_{[+]}^{\Lambda}(\mathbf{z})=Y_{+}^{\Lambda} \mathbf{z}^{-1}+\sum_{n=0}^{\infty} \oint_{0} \frac{d \mathbf{z}^{\prime}}{2 \pi \mathrm{i} \mathbf{z}^{\prime}}\left(\frac{\mathbf{z}}{\mathbf{z}^{\prime}}\right)^{n} \xi_{[+]}^{\Lambda}\left(\mathbf{z}^{\prime}\right) .
$$

The contour around $\mathbf{z}=0$ may be deformed into a sum of contours $\tilde{C}_{j}$ around the other singularities in the $\mathbf{z}$ plane. Using the contact transformations (5.23) on each patch, we obtain

$$
\xi_{[+]}^{\Lambda}(\mathbf{z})=Y_{+}^{\Lambda} \mathbf{z}^{-1}-\sum_{n=0}^{\infty} \sum_{j \neq+} \oint_{\tilde{C}_{j}} \frac{d \mathbf{z}^{\prime}}{2 \pi \mathrm{i} \mathbf{z}^{\prime}}\left(\frac{\mathbf{z}}{\mathbf{z}^{\prime}}\right)^{n}\left[\xi_{[j]}^{\Lambda}\left(\mathbf{z}^{\prime}\right)-T_{[+j]}^{\Lambda}\left(\mathbf{z}^{\prime}\right)\right] .
$$

The first term in the square bracket gives a non-vanishing contribution for $j=-$ and $n=0,1$ only, while the second term contributes an infinite Laurent series. Therefore, we arrive at the following representation:

$$
\xi_{[+]}^{\Lambda}(\mathbf{z})=Y_{+}^{\Lambda} \mathbf{z}^{-1}+A_{-}^{\Lambda}-Y_{-}^{\Lambda} \mathbf{z}+\sum_{j} \oint_{\tilde{C}_{j}} \frac{d \mathbf{z}^{\prime}}{2 \pi \mathrm{i}} \frac{1}{\mathbf{z}^{\prime}-\mathbf{z}} T_{[+j]}^{\Lambda}\left(\mathbf{z}^{\prime}\right),
$$

where now

$$
A_{-}^{\Lambda}=-\oint_{\infty} \frac{\mathrm{d} \mathbf{z}}{2 \pi \mathrm{i} \mathbf{z}} \xi_{[-]}^{\Lambda}, \quad Y_{-}^{\Lambda}=\oint_{\infty} \frac{\mathrm{d} \mathbf{z}}{2 \pi \mathrm{i} \mathbf{z}^{2}} \xi_{[-]}^{\Lambda} .
$$

From the reality conditions (2.70), we conclude that $A_{-}=\left(A_{+}\right)^{*}, Y_{-}^{\Lambda}=\left(Y_{+}^{\Lambda}\right)^{*}$. Comparing the $\mathcal{O}\left(\mathbf{z}^{0}\right)$ terms between (5.24) and (5.28) gives the difference

$$
A_{+}^{\Lambda}-A_{-}^{\Lambda}=\sum_{j} \oint_{\tilde{C}_{j}} \frac{\mathrm{d} \mathbf{z}^{\prime}}{2 \pi \mathrm{i} \mathbf{z}^{\prime}} T_{[+j]}^{\Lambda}\left(\mathbf{z}^{\prime}\right) .
$$

Eliminating $A_{-}^{\Lambda}$ in (5.28) in favor of the real quantity $A^{\Lambda}=\left(A_{+}^{\Lambda}+A_{-}^{\Lambda}\right) / 2$ leads to

$$
\xi_{[i]}^{\Lambda}(\mathbf{z})=A^{\Lambda}+\left(\mathbf{z}^{-1} Y_{+}^{\Lambda}-\mathbf{z} Y_{-}^{\Lambda}\right)+\frac{1}{2} \sum_{j} \oint_{\tilde{C}_{j}} \frac{d \mathbf{z}^{\prime}}{2 \pi \mathrm{i} \mathbf{z}^{\prime}} \frac{\mathbf{z}^{\prime}+\mathbf{z}}{\mathbf{z}^{\prime}-\mathbf{z}} T_{[+j]}^{\Lambda}\left(\mathbf{z}^{\prime}\right)
$$

25 The twisting by $\mathcal{O}(2)$ is not apparent in our formalism, as explained in footnote 14, but follows from the fact that $\hat{H}_{(i)}^{[i j]}$ originates from a homogeneous function of degree 1 on $\mathcal{S}$. 
for $i=+$. As observed below (3.53), these equations are in fact valid in any patch $\mathcal{U}_{i}$, since they exhibit the correct discontinuities across the contours $\tilde{C}_{i}$.

In an analogous way one may obtain the deformed conjugate coordinates $\tilde{\xi}_{I}^{[i]}$. The Laurent coefficient $\tilde{\xi}_{I, 0}^{[+]}$may be extracted by integrating

$$
\tilde{\xi}_{I, 0}^{[+]}=\oint_{0} \frac{\mathrm{d} \mathbf{z}^{\prime}}{2 \pi \mathrm{i} \mathbf{z}^{\prime}}\left(\tilde{\xi}_{I}^{[+]}-c_{I}^{[+]} \log \mathbf{z}^{\prime}\right)=\mathrm{i} B_{I}^{+}-c_{I}^{[+]} \oint_{0} \frac{\mathrm{d} \mathbf{z}^{\prime}}{2 \pi \mathrm{i} \mathbf{z}^{\prime}} \log \left(\mathbf{z}^{\prime} \xi_{[+]}^{0}\right),
$$

where we defined

$$
B_{I}^{+} \equiv-\mathrm{i} \oint_{0} \frac{\mathrm{d} \mathbf{z}^{\prime}}{2 \pi \mathrm{i} \mathbf{z}^{\prime}}\left(\mu_{I}^{[+]}+c_{I}^{[+]} \log \nu_{[+]}^{0}\right) .
$$

On the other hand, the Laurent series expansion around $\mathbf{z}=0$ may be obtained by deforming the contour around $\mathbf{z}=0$ into a sum of contours around the other singularities in the $\mathbf{z}$ plane, and use the symplectomorphism (5.22) to map $\tilde{\xi}_{I}^{[+]}$to $\tilde{\xi}_{I}^{[j]}$ :

$$
\tilde{\xi}_{I}^{[+]}(\mathbf{z})=c_{I}^{[+]} \log \mathbf{z}-\sum_{n=0}^{\infty} \sum_{j \neq+} \oint_{\tilde{C}_{j}} \frac{\mathrm{d} \mathbf{z}^{\prime}}{2 \pi \mathrm{i} \mathbf{z}^{\prime}}\left(\frac{\mathbf{z}}{\mathbf{z}^{\prime}}\right)^{n}\left(\tilde{\xi}_{I}^{[j]}-c_{I}^{[+]} \log \mathbf{z}^{\prime}-\tilde{T}_{I}^{[+j]}\right),
$$

where $\tilde{T}_{I}^{[i j]}$ are defined in (5.23). The first two terms in the bracket only contribute when $j=-$. The cancelation of the logarithmic singularity at $\mathbf{z}=\infty$ is ensured by the condition $c_{I}^{[+]}=-c_{I}^{[-]}$, corresponding to the figure-eight contour prescription discussed in [1]. Using (2.81), we obtain

$$
\tilde{\xi}_{I}^{[+]}(\mathbf{z})=c_{I}^{[+]} \log \mathbf{z}+\mathrm{i} B_{I}^{-}+c_{I}^{[-]} \oint_{\infty} \frac{\mathrm{d} \mathbf{z}^{\prime}}{2 \pi \mathrm{i} \mathbf{z}^{\prime}} \log \frac{\xi_{[-]}^{0}}{\mathbf{z}^{\prime}}+\sum_{j} \oint_{\tilde{C}_{j}} \frac{\mathrm{d} \mathbf{z}^{\prime}}{2 \pi \mathrm{i}\left(\mathbf{z}^{\prime}-\mathbf{z}\right)} \tilde{T}_{I}^{[+j]},
$$

where

$$
B_{I}^{-} \equiv \mathrm{i} \oint_{\infty} \frac{\mathrm{d} \mathbf{z}^{\prime}}{2 \pi \mathrm{i} \mathbf{z}^{\prime}}\left(\mu_{I}^{[-]}+c_{I}^{[-]} \log \nu_{[-]}^{0}\right)
$$

is the complex conjugate of $B_{I}^{+}$. Comparing the $\mathbf{z}$-independent terms in (5.34) and (5.35) establishes the identity

$$
\begin{aligned}
\mathrm{i}\left(B_{I}^{+}-B_{I}^{-}\right)= & c_{I}^{[+]} \oint_{0} \frac{\mathrm{d} \mathbf{z}^{\prime}}{2 \pi \mathrm{i} \mathbf{z}^{\prime}} \log \mathbf{z}^{\prime} \xi_{[+]}^{0}-c_{I}^{[-]} \oint_{\infty} \frac{\mathrm{d} \mathbf{z}^{\prime}}{2 \pi \mathrm{i} \mathbf{z}^{\prime}} \log \frac{\mathbf{z}^{\prime}}{\xi_{[-]}^{0}} \\
& +\sum_{j} \oint_{\tilde{C}_{j}} \frac{\mathrm{d} \mathbf{z}^{\prime}}{2 \pi \mathrm{i} \mathbf{z}^{\prime}} \tilde{T}_{I}^{[+j]} .
\end{aligned}
$$

Eliminating $B_{I}^{-}$in (5.35) in favor of $B_{I}=B_{I}^{+}+B_{I}^{-}$leads to

$$
\begin{aligned}
\tilde{\xi}_{I}^{[+]}(\mathbf{z})= & \frac{\mathrm{i}}{2} B_{I}-\frac{1}{2} c_{I}^{[+]}\left[\oint_{0} \frac{\mathrm{d} \mathbf{z}^{\prime}}{2 \pi \mathrm{i} \mathbf{z}^{\prime}} \log \left(\mathbf{z}^{\prime} \xi_{[+]}^{0}\right)+\oint_{\infty} \frac{\mathrm{d} \mathbf{z}^{\prime}}{2 \pi \mathrm{i} \mathbf{z}^{\prime}} \log \left(\xi_{[-]}^{0} / \mathbf{z}^{\prime}\right)\right] \\
& +\frac{1}{2} \sum_{j} \oint_{\tilde{C}_{j}} \frac{\mathrm{d} \mathbf{z}^{\prime}}{2 \pi \mathrm{i} \mathbf{z}^{\prime}} \frac{\mathbf{z}^{\prime}+\mathbf{z}}{\mathbf{z}^{\prime}-\mathbf{z}} \tilde{T}_{I}^{[+j]}+c_{I}^{[+]} \log \mathbf{z} .
\end{aligned}
$$


Using the fact that $\xi_{[+]}^{0} \sim Y_{+}^{0} / \mathbf{z}^{\prime}$ and $\xi_{[-]}^{0} \sim Y_{-}^{0} \mathbf{z}^{\prime}$ at $\mathbf{z}^{\prime}=0$ and $\infty$, we finally obtain

$$
\tilde{\xi}_{I}^{[i]}(\mathbf{z})=\frac{\mathrm{i}}{2} B_{I}+\frac{1}{2} \sum_{j} \oint_{\tilde{C}_{j}} \frac{\mathrm{d} \mathbf{z}^{\prime}}{2 \pi \mathrm{i} \mathbf{z}^{\prime}} \frac{\mathbf{z}^{\prime}+\mathbf{z}}{\mathbf{z}^{\prime}-\mathbf{z}} \tilde{T}_{I}^{[+j]}+c_{I}^{[+]} \log \left(\mathbf{z} \sqrt{\frac{Y_{-}^{0}}{Y_{+}^{0}}}\right)
$$

for $i=+$, and in fact also for any $i$. This relation generalizes (3.53) to the perturbed case. Taken together, (5.31) and (5.39) give the contact twistor lines of the deformed twistor space in terms of the perturbation $H_{(1)}^{[i j]}$, which is considered as a function of the undeformed twistor lines $\breve{\xi}_{[i]}^{\Lambda}, \breve{\xi}_{I}^{[i]}$ given in (3.20) and (3.53). In order to make contact with the construction in Sect. 3.3, one should recall that $Y_{+}^{\Lambda}=\mathcal{R} Z^{\Lambda}$, where

$$
Z^{\Lambda}=\oint_{0} \frac{\mathrm{d} \mathbf{z}}{2 \pi \mathrm{i} \mathbf{z}} \frac{\xi_{[+]}^{\Lambda}}{\xi_{[+]}^{0}}, \quad \mathcal{R}=\oint_{0} \frac{\mathrm{d} \mathbf{z}}{2 \pi \mathrm{i}} \xi_{[+]}^{0},
$$

in such a way that $Z^{0}=1$.

To obtain the perturbed quaternionic-Kähler metric, we should also calculate the leading Laurent coefficients of the contact potentials $\Phi_{[ \pm]}$given in (2.84). It turns out that one can actually compute the full contact potentials, using the gluing conditions

$$
e^{-\Phi_{[i]}}-e^{-\Phi_{[j]}}=e^{-\phi} \partial_{\tilde{\xi}_{b}^{[j]}} \hat{H}_{(1)}^{[i j]},
$$

where $\phi$ is defined by (3.38) in the unperturbed geometry. These conditions follow from the gluing conditions for $\tilde{v}_{[i]}^{b}$ in (2.69), using the results for the transition functions $\hat{f}_{i j}^{2}$ (5.21) and the unperturbed $\tilde{v}_{[i]}^{b}$ (3.45). Repeating again the same steps as above, one easily arrives at

$$
e^{\Phi_{[i]}}=e^{\phi}\left(1+\frac{1}{2} \sum_{j} \oint_{\tilde{C}_{j}} \frac{\mathrm{d} \mathbf{z}^{\prime}}{2 \pi \mathrm{i} \mathbf{z}^{\prime}} \frac{\mathbf{z}^{\prime}+\mathbf{z}}{\mathbf{z}^{\prime}-\mathbf{z}} \partial_{\tilde{\xi}_{b}^{[j]}} \hat{H}_{(1)}^{[0 j]}\left(\mathbf{z}^{\prime}\right)\right),
$$

where $\phi$ is defined in the perturbed case as

$$
\phi \equiv \operatorname{Re} \Phi_{[+]}(\mathbf{z}=0)=\frac{1}{2}\left(\phi_{[+]}^{0}+\phi_{[-]}^{0}\right) .
$$

Note that this definition coincides with (3.38) in the unperturbed case. Using (2.84), the leading Laurent coefficient of the contact potentials are given by

$$
\begin{aligned}
& e^{\phi_{[+]}^{0}}=\frac{1}{2} Y_{+}^{\Lambda} \sum_{j} \oint_{\tilde{C}_{j}} \frac{d \mathbf{z}}{2 \pi \mathrm{i} \mathbf{z}^{2}} \tilde{T}_{\Lambda}^{[0 j]}+\frac{1}{2} c_{\Lambda}^{[+]}\left(A^{\Lambda}+\frac{1}{2} \sum_{j} \oint_{\tilde{C}_{j}} \frac{d \mathbf{z}}{2 \pi \mathrm{i} \mathbf{z}} T_{[0 j]}^{\Lambda}\right)+\frac{1}{2} c_{b}^{[+]}, \\
& e^{\phi_{[-]}^{0}}=-\frac{1}{2} Y_{-}^{\Lambda} \sum_{j} \oint_{\tilde{C}_{j}} \frac{d \mathbf{z}}{2 \pi \mathrm{i}} \tilde{T}_{\Lambda}^{[0 j]}-\frac{1}{2} c_{\Lambda}^{[-]}\left(A^{\Lambda}-\frac{1}{2} \sum_{j} \oint_{\tilde{C}_{j}} \frac{d \mathbf{z}}{2 \pi \mathrm{i} \mathbf{z}} T_{[0 j]}^{\Lambda}\right)-\frac{1}{2} c_{b}^{[-]} .
\end{aligned}
$$

Inserting in (5.43) we therefore obtain

$$
e^{\phi}=\frac{1}{4} \sum_{j} \oint_{\tilde{C}_{j}} \frac{d \mathbf{z}}{2 \pi \mathrm{i} \mathbf{z}}\left(\mathbf{z}^{-1} Y_{+}^{\Lambda}-\mathbf{z} Y_{-}^{\Lambda}\right) \tilde{T}_{\Lambda}^{[0 j]}+\frac{1}{4} c_{I}^{[+-]} A^{I},
$$


and the full contact potentials via (5.42). As a useful consistency check, note that the difference of (5.44) can be rewritten, after some considerable work, as

$$
\phi_{[+]}^{0}-\phi_{[-]}^{0}=\sum_{j} \oint_{\tilde{C}_{j}} \frac{\mathrm{d} \mathbf{z}}{2 \pi \mathrm{i} \mathbf{z}} \partial_{\tilde{\xi}_{b}^{[j]}} \hat{H}_{(1)}^{[0 j]}
$$

consistently with (5.42). Altogether these results allow us to extract the metric following the procedure outlined at the end of Sect. 2.5.

It is straightforward to relate this contact construction on $\mathcal{Z}_{\mathcal{M}}$ to the symplectic construction on $\mathcal{Z}_{\mathcal{S}}$. For this purpose, one needs to apply the change of variable (2.61), where $\zeta_{ \pm}$denote the location of the zeros of the perturbed section $v^{b}$, to all contour integrals in the $\mathbf{z}$ plane. Under this change of variable, the integration measure becomes

$$
\frac{\mathrm{d} \mathbf{z}}{2 \pi \mathrm{i} \mathbf{z}}=\frac{\left(\zeta_{+}-\zeta_{-}\right)}{\left(\zeta-\zeta_{+}\right)\left(\zeta-\zeta_{-}\right)} \frac{\mathrm{d} \zeta}{2 \pi \mathrm{i}}
$$

Its expression to the first order in deformation can be found in (C.9). However, in some cases it can be simplified. For example, integrated against a function which is regular at $\zeta_{ \pm}$, this may be rewritten as

$$
\frac{\mathrm{d} \mathbf{z}}{2 \pi \mathrm{i} \mathbf{z}}=\frac{r_{ \pm}^{\mathrm{b}} \mathrm{d} \zeta}{2 \pi \mathrm{i} f_{0 \pm}^{2} \nu_{[ \pm]}^{\mathrm{b}}}, \quad \frac{1}{r_{ \pm}^{\mathrm{b}}} \equiv \oint_{C_{+}} \frac{\mathrm{d} \zeta}{2 \pi \mathrm{i} f_{0 \pm}^{2} \nu_{[ \pm]}^{b}},
$$

where the factor $r_{ \pm}^{b}$ ensures that the residue at $\zeta=\zeta_{ \pm}$is equal to one. Integrating a function with a simple pole at $\zeta_{ \pm},(5.48)$ must be generalized to

$$
\frac{\mathrm{d} \mathbf{z}}{2 \pi \mathrm{i} \mathbf{z}}=\frac{\mathrm{d} \zeta}{2 \pi \mathrm{i}}\left[\frac{r_{ \pm}^{\mathrm{b}}}{f_{0 \pm}^{2} \nu_{[ \pm]}^{\mathrm{b}}}+\left(\frac{1}{\zeta_{-}-\zeta_{+}}+\frac{s_{ \pm}^{\mathrm{b}}}{r_{ \pm}^{\mathrm{b}}}\right)\right],
$$

where $s_{ \pm}^{b}$ is the second coefficient in the Taylor expansion

$$
f_{0 \pm}^{2} v_{[ \pm]}^{b}=r_{ \pm}^{b}\left(\zeta-\zeta_{ \pm}\right)+s_{ \pm}^{b}\left(\zeta-\zeta_{ \pm}\right)^{2}+\cdots
$$

Note that the correction term in round brackets in (5.49) vanishes in the case where $v^{b}$ remains a global $\mathcal{O}(2)$ section. In this way, we may rewrite the invariant coordinates introduced above as follows:

$$
\begin{aligned}
& Y_{ \pm}^{\Lambda} \equiv r_{ \pm}^{b} \oint_{C_{ \pm}} \frac{\mathrm{d} \zeta}{2 \pi \mathrm{i} f_{0 \pm}^{2}} \frac{v_{[ \pm]}^{\Lambda}}{\left(v_{[ \pm]}^{\mathrm{b}}\right)^{2}} \mathbf{z}^{ \pm 1} \\
& A_{ \pm}^{\Lambda} \equiv \pm \oint_{C_{ \pm}} \frac{\mathrm{d} \zeta}{2 \pi \mathrm{i}}\left[\frac{r_{ \pm}^{b}}{f_{0 \pm}^{2} v_{[ \pm]}^{b}}+\left(\frac{1}{\zeta_{-}-\zeta_{+}}+\frac{s_{ \pm}^{b}}{r_{ \pm}^{b}}\right)\right] \frac{v_{[ \pm]}^{\Lambda}}{v_{[ \pm]}^{b}} \\
& B_{I}^{ \pm} \equiv \mp \mathrm{i} r_{ \pm}^{b} \oint_{C_{ \pm}} \frac{\mathrm{d} \zeta}{2 \pi \mathrm{i} f_{0 \pm}^{2} v_{[+]}^{b}}\left(\mu_{I}^{[ \pm]}+c_{I}^{[ \pm]} \log v_{[ \pm]}^{0}\right)
\end{aligned}
$$

From the computation of the deformed hyperkähler potential (C.22) in Appendix C, one may check that the relation (3.38) continues to hold after perturbation, provided $\phi$ 
is defined by (5.43) and $r^{b}=\left(r_{+}^{b}+r_{-}^{b}\right) / 2$. From the general equation (2.78), this implies that

$$
r^{b}=\left|v^{b} \hat{f}_{0+}^{2}\right| \frac{1+z \bar{z}}{|z|} e^{\operatorname{Re}\left[\Phi_{[+]}\left(x^{\mu}, z\right)-\phi_{[+]}^{0}\left(x^{\mu}\right)\right]} .
$$

We have not attempted to check this relation directly.

In [19], we shall apply this general framework to the hypermultiplet moduli space in compactifications of type II string theory on a Calabi-Yau three-fold.

Acknowledgements. We are grateful to A. Neitzke for discussions and former collaboration on related topics. The research of S.A. is supported by CNRS and by the contract ANR-05-BLAN-0029-01. The research of B.P. is supported in part by ANR(CNRS-USAR) contract no.05-BLAN-0079-01. F.S. acknowledges financial support from the ANR grant BLAN06-3-137168. S.V. thanks the Federation de Recherches "Interactions Fondamentales" and LPTHE at Jussieu for hospitality and financial support. Part of this work is also supported by the EU-RTN network MRTN-CT-2004-005104 "Constituents, Fundamental Forces and Symmetries of the Universe".

Open Access This article is distributed under the terms of the Creative Commons Attribution Noncommercial License which permits any noncommercial use, distribution, and reproduction in any medium, provided the original author(s) and source are credited.

\section{A. Infinitesimal $S U$ (2) Transformations}

In this appendix, we study the infinitesimal action of $S U(2)$ on the local sections introduced in the main text. We parametrize the Lie algebra of $S U(2)$ by $\epsilon_{ \pm}=\left(\epsilon_{\mp}\right)^{*}$ and $\epsilon_{3}=\left(\epsilon_{3}\right)^{*}$ such that

$$
\left(\begin{array}{cc}
\alpha & \beta \\
-\bar{\beta} & \bar{\alpha}
\end{array}\right)=\left(\begin{array}{cc}
1-\frac{\mathrm{i}}{2} \epsilon_{3} & \epsilon_{+} \\
-\epsilon_{-} & 1+\frac{\mathrm{i}}{2} \epsilon_{3}
\end{array}\right)+\mathcal{O}\left(\epsilon^{2}\right) .
$$

The infinitesimal action of $S U(2)$ on $\pi^{A^{\prime}}, \bar{\pi}_{A^{\prime}}$ is given in (2.4),

$$
\delta\left(\begin{array}{cc}
\pi^{1} & \pi^{2} \\
-\bar{\pi}_{2} & \bar{\pi}_{1}
\end{array}\right)=\left(\begin{array}{cc}
\frac{i}{2} \epsilon_{3} & -\epsilon_{+} \\
\epsilon_{-} & -\frac{i}{2} \epsilon_{3}
\end{array}\right) \cdot\left(\begin{array}{cc}
\pi^{1} & \pi^{2} \\
-\bar{\pi}_{2} & \bar{\pi}_{1}
\end{array}\right) .
$$

The finite action of $S U(2)$ on $\mathcal{O}(2 n)$ sections was discussed in Sect. 2.4. At the infinitesimal level, (2.41) reduces to

$$
\delta \zeta \equiv \zeta^{\prime}-\zeta=\epsilon_{+}-\mathrm{i} \epsilon_{3} \zeta+\epsilon_{-} \zeta^{2}+\mathcal{O}\left(\epsilon^{2}\right) .
$$

In the patch $i=0$, the $\mathcal{O}(2 n)$ transformation rule (2.44) then leads to

$$
\begin{aligned}
\delta v_{[0]}^{I}(\zeta) & \equiv v_{[0]}^{\prime I}(\zeta)-v_{[0]}^{I}(\zeta) \\
& =\left[\epsilon_{+} \partial_{\zeta}-\mathrm{i} \epsilon_{3}\left(\zeta \partial_{\zeta}-n\right)+\epsilon_{-}\left(\zeta^{2} \partial_{\zeta}-2 n \zeta\right)\right] v_{[0]}^{I}(\zeta)+\mathcal{O}\left(\epsilon^{2}\right)
\end{aligned}
$$

Thus, the Taylor coefficients of $\nu_{[0]}=\sum_{m} v_{m}^{I} \zeta^{m}$ around $\zeta=0$ vary under an infinitesimal $S U(2)$ action by

$$
\delta v_{m}^{I}=(m+1) v_{m+1}^{I} \epsilon_{+}-\mathrm{i}(m-n) v_{m}^{I} \epsilon_{3}+(m-2 n-1) v_{m-1}^{I} \epsilon_{-} .
$$

The variation of $\mu_{I}^{[0]}$ and its Laurent coefficients $\mu_{I, m}$ is obtained by replacing $v^{I} \rightarrow$ $\mu_{I}, n \rightarrow 1-n$ in these expressions. 
In an arbitrary patch $\mathcal{U}_{i}$, the $S U(2)$ action (2.44) is most easily expressed in terms of $f_{i 0}^{-2 n}(\zeta) v_{[i]}^{I}(\zeta)$, which formally transforms in the same way as $v_{[0]}^{I}(\zeta)$. Similarly, the $S U(2)$ action (2.54) is most easily stated in terms of $f_{i 0}^{-2}(\zeta) \exp \left(-\mu^{[i]} / c_{I}^{[i]}\right)$, which also formally transforms in the same way as $v_{[0]}^{I}(\zeta)$. After the gauge transformation (3.13), the transformation rules of $\mu_{I}^{[i]}$ are changed to

$$
\begin{aligned}
\delta \mu_{T ; I}^{[i]}(\zeta)= & \left(\epsilon_{+}-\mathrm{i} \epsilon_{3} \zeta+\epsilon_{-} \zeta^{2}\right) \partial_{\zeta} \mu_{T ; I}^{[i]}(\zeta)+\left(\frac{\epsilon_{+}}{\zeta}-\mathrm{i} \epsilon_{3}+\epsilon_{-} \zeta\right) c_{I}^{[0]} \\
& -\left(\frac{\epsilon_{+}}{\zeta}-\epsilon_{-} \zeta\right) c_{I}^{[i]}
\end{aligned}
$$

consistently with the fact that (3.18) transforms like a non-anomalous $\mathcal{O}(0)$ section.

The variation (A.5) applies for the Laurent coefficients of any local section of $\mathcal{O}(2 n)$. In particular, one may consider a homogeneous function $G\left(v_{\alpha}\right)$ of $\mathcal{O}\left(2 n_{\alpha}\right)$ multiplets $v_{\alpha}$, of homogeneity degree $n$ when each $v_{\alpha}$ are scaled with homogeneity degree $n_{\alpha}$ :

$$
\sum_{\alpha} n_{\alpha} v_{\alpha} \partial_{\nu_{\alpha}} G=n G
$$

Then, for an arbitrary contour $\Gamma$ (not necessarily surrounding the origin), the $S U(2)$ variation of the integrals

$$
G_{m} \equiv \oint_{\Gamma} \frac{\mathrm{d} \zeta}{2 \pi \mathrm{i} \zeta^{m+1}} G\left(v_{\alpha}\right)
$$

is given by

$$
\delta G_{m}=(m+1) G_{m+1} \epsilon_{+}-\mathrm{i}(m-n) G_{m} \epsilon_{3}+(m-1-2 n) G_{m-1} \epsilon_{-},
$$

as one can check from explicit calculation. In particular, for $n=m=-1$, we recover the remark in [39], according to which a contour integral of a section of $\mathcal{O}(-2)$ is $S U(2)$ invariant. For $n=-3 / 2$, the contour integral of a section of $\mathcal{O}(-3)$ with $m=-1,-2$ instead produces a $S U(2)$ doublet. These observations are central to the superconformal quotient discussed in Sect. 3.2.

\section{B. An alternative Formulation for Hypermultiplet Moduli Spaces}

In this appendix we explain the relation between the formulation of the hypermultiplet space used in Sect. 4, and the one introduced in [28] using a different contour prescription, and establish their equivalence up to a local symplectomorphism.

Aiming for a Lagrangian $\mathcal{L}^{\prime}$ whose limit $v^{b} \rightarrow 0$ is regular, [28] considered the contour integral

$$
\mathcal{L}^{\prime}(v, \bar{v}, x)=\operatorname{Im} \oint_{C} \frac{\mathrm{d} \zeta}{2 \pi \mathrm{i} \zeta}\left(\frac{F\left(\eta^{\Lambda}\right)}{\eta^{b}}+4 \mathrm{i} c \eta^{b} \log \eta^{b}\right),
$$


where the contour $C$ appearing in (4.1) encircles the poles $\zeta=0, \zeta_{+}$in the counter-clockwise direction and the logarithmic branch cuts connect $0, \zeta_{+}$and $\zeta_{-}, \infty$, respectively. The resulting Lagrangian

$$
\begin{aligned}
\mathcal{L}^{\prime}= & \frac{1}{r^{b}} \operatorname{Im} F\left(\eta_{+}\right)-x^{b} \operatorname{Im} \frac{F(v)}{\left(v^{b}\right)^{2}}+x^{\Lambda} \operatorname{Im} \frac{F_{\Lambda}(v)}{v^{b}} \\
& +4 c\left(x^{b}-r^{b}+x^{b} \log \frac{x^{b}+r^{b}}{2}\right)
\end{aligned}
$$

differs from (4.3), (4.14) by terms linear in $x^{I}$ only and therefore describes the same metric. In terms of our general discussion, the contour prescription (B.1) arises from the transition functions

$$
\begin{aligned}
& H^{\prime[0+]}=0, \quad H^{\prime}[0 i]=\frac{\mathrm{i}}{2} \frac{F(\eta)}{\eta^{\mathrm{b}}}, \\
& H^{\prime[0-]}=H^{\prime[0 \infty]}=\frac{\mathrm{i}}{2} \frac{F(\eta)-\bar{F}(\eta)}{\eta^{\mathrm{b}}}-4 c \eta^{\mathrm{b}} \log \eta^{\mathrm{b}},
\end{aligned}
$$

where $i$ labels the patches where $F(\eta)$ is singular. These transition functions are related to the ones given in (4.2), (4.15) by the gauge transformation generated by

$$
\begin{aligned}
G^{[0]} & =\frac{\mathrm{i}}{2} \frac{F(\eta)}{\eta^{b}}-2 c \eta^{\mathrm{b}} \log \left(\eta^{\mathrm{b}} \zeta\right), \quad G^{[\infty]}=\frac{\mathrm{i}}{2} \frac{\bar{F}(\eta)}{\eta^{b}}+2 c \eta^{b} \log \left(\eta^{\mathrm{b}} / \zeta\right), \\
G^{[+]} & =G^{[-]}=G^{[i]}=-2 c \eta^{b} \log (\zeta) .
\end{aligned}
$$

The new non-vanishing quasi-homogeneity coefficients (3.16) are

$$
c_{\mathrm{b}}^{[0]}=c_{\mathrm{b}}^{[+]}=-2 c, \quad c_{\mathrm{b}}^{[\infty]}=c_{\mathrm{b}}^{[-]}=2 c .
$$

Note that in contrast to the description in Sect. 4.1, the coefficient $c_{b}^{[0]}$ does not vanish, corresponding to the Lagrangian (B.2) being quasi-homogeneous.

We now discuss the twistor lines arising from this new contour prescription. The global $\mathcal{O}(2)$ sections $\eta(\zeta)$ are unchanged while the gauge transformation (B.4) induces

$$
\begin{aligned}
& \mu_{\Lambda}^{\prime[0]}(\zeta)=\mu_{\Lambda}^{[0]}(\zeta)-\frac{\mathrm{i}}{2} \frac{F_{\Lambda}(\eta)}{\eta^{\mathrm{b}}} \\
& \mu_{\mathrm{b}}^{\prime[0]}(\zeta)=\mu_{\mathrm{b}}^{[0]}(\zeta)+\frac{\mathrm{i}}{2} \frac{F(\eta)}{\left(\eta^{b}\right)^{2}}+2 c\left(\log \left(\eta^{\mathrm{b}} \zeta\right)+1\right)
\end{aligned}
$$

Consequently, the coordinates $w_{I}$ become

$$
w_{\Lambda}^{\prime}=w_{\Lambda}-\frac{\mathrm{i}}{2} \frac{F_{\Lambda}(v)}{v^{b}}, \quad w_{b}^{\prime}=w_{b}+\frac{\mathrm{i}}{2} \frac{F(v)}{\left(v^{b}\right)^{2}}+2 c\left(\log \left(v^{b}\right)+1\right) .
$$

The corresponding map between the coordinates $\varrho_{I}$ and $\varrho_{I}^{\prime}$ is readily obtained using $\varrho_{I}=-\mathrm{i}\left(w_{I}-\bar{w}_{I}\right)$. Furthermore, the base coordinates (3.36)-(3.39) are identical,

$$
e^{\phi^{\prime}}=e^{\phi}, \quad Z^{\prime a}=Z^{a}, \quad A^{\prime \Lambda}=A^{\Lambda}, \quad B_{I}^{\prime}=B_{I},
$$

where the last equality follows from (3.39) upon a brief computation. Taking into account also that only $c_{I}^{[+-]}$, which are equal in the two formulations, contribute to the general expressions (3.53), this, in turn, implies that both contour prescriptions give rise to the same twistor lines (4.23). 


\section{Deformed Superconformal Quotient}

In this appendix we generalize the superconformal quotient procedure of Sect. 3.2 to include deformations. While conceptually straightforward, this procedure is toilsome compared to the contact geometry approach of Sect. 5.2. Nevertheless, we include it here for completeness, as it provides useful consistency checks on our formalism,

Coordinates on the deformed base. As in the undeformed case described in Sect. 3.2, $S U$ (2) invariant functions on the Swann bundle $\mathcal{S}$ can be obtained by contour-integrating $\mathcal{O}(-2)$ sections on $\mathcal{Z}_{\mathcal{S}}$. In the presence of deformations, the global sections $\eta^{I}$ in (3.36) must be replaced by the deformed local sections $v_{[+]}^{I}$, leading to the definitions ${ }^{26}$

$$
\begin{gathered}
\frac{1}{r^{b}} \equiv \operatorname{Re} \oint_{C_{+}} \frac{\mathrm{d} \zeta}{2 \pi \mathrm{i} f_{0+}^{2}} \frac{1}{v_{[+]}^{\mathrm{b}}}, \quad \mathcal{A}^{\Lambda} \equiv r^{\mathrm{b}} \operatorname{Re} \oint_{C_{+}} \frac{\mathrm{d} \zeta}{2 \pi \mathrm{i} f_{0+}^{2}} \frac{v_{[+]}^{\Lambda}}{\left(v_{[+]}^{b}\right)^{2}} \\
\mathcal{Z}^{a} \equiv r^{\mathrm{b}} \oint_{C_{+}} \frac{\mathrm{d} \zeta}{2 \pi \mathrm{i} f_{0+}^{2}} \frac{v_{[+]}^{a}}{v_{[+]}^{\mathrm{b}} v_{[+]}^{0}}, \quad \mathcal{B}_{I} \equiv 2 r^{\mathrm{b}} \operatorname{Im} \oint_{C_{+}} \frac{\mathrm{d} \zeta}{2 \pi \mathrm{i} f_{0+}^{2}} \frac{\mu_{I}^{[+]}+c_{I}^{[+]} \log v_{[+]}^{0}}{v_{[+]}^{b}}
\end{gathered}
$$

To first order in the deformation, this gives

$$
\begin{aligned}
r^{b}= & \breve{r}^{b}+\frac{1}{2}\left(\hat{v}_{\zeta,+}^{b}-\hat{v}_{\zeta,-}^{b}\right)+\frac{\bar{v}^{b}}{r^{b}}\left(\hat{v}_{+}^{b}+\hat{v}_{-}^{b}\right), \\
\mathcal{Z}^{a}= & \breve{Z}^{a}+\frac{\hat{v}_{+}^{a}-\breve{A}^{a} \hat{v}_{+}^{b}}{\zeta_{+} \eta_{+}^{0}}-\frac{\hat{v}_{+}^{0}-\breve{A}^{0} \hat{v}_{+}^{b}}{\zeta_{+} \eta_{+}^{0}} \breve{Z}^{a}, \\
\mathcal{A}^{\Lambda}= & \breve{A}^{\Lambda}+\frac{1}{2 r^{b}}\left(\hat{v}_{\zeta,+}^{\Lambda}-\hat{v}_{\zeta,-}^{\Lambda}+\frac{2 \bar{v}^{b}}{r^{b}}\left(\hat{v}_{+}^{\Lambda}+\hat{v}_{-}^{\Lambda}\right)\right) \\
& -\frac{1}{\left(r^{b}\right)^{2}} \operatorname{Re}\left[\zeta_{+} \eta_{+}^{\Lambda} \hat{v}_{\zeta \zeta,+}^{b}+\left(2 r^{b} \breve{A}^{\Lambda}-x^{\Lambda}+2 \bar{v}^{\Lambda} \zeta_{+}\right) \hat{v}_{\zeta,+}^{b}+2\left(2 \bar{v}^{b} \breve{A}^{\Lambda}-\bar{v}^{\Lambda}\right) \hat{v}_{+}^{b}\right],
\end{aligned}
$$

where ${ }^{`}$ marks the unperturbed quantities defined in (3.36) and we introduced

$$
\hat{v}_{ \pm}^{I}=f_{0 \pm}^{2} \hat{v}_{[ \pm]}^{I}\left(\zeta_{ \pm}\right), \quad \hat{v}_{\zeta, \pm}^{I}=\partial_{\zeta}\left(f_{0 \pm}^{2} \hat{v}_{[ \pm]}^{I}\right)\left(\zeta_{ \pm}\right), \quad \hat{v}_{\zeta \zeta, \pm}^{I}=\partial_{\zeta}^{2}\left(f_{0 \pm}^{2} \hat{v}_{[ \pm]}^{I}\right)\left(\zeta_{ \pm}\right)
$$

We omitted the expansion of $\mathcal{B}_{I}$ since it will not be needed. The $S U(2)$ invariant $\mathcal{R}$ defined in (3.40) may also be extended to the deformed case as

$$
\begin{aligned}
\mathcal{R}= & \breve{\mathcal{R}}\left[1+\frac{1}{2}\left(\frac{\hat{v}_{+}^{0}-\breve{A}^{0} \hat{v}_{+}^{b}}{\zeta_{+} \eta_{+}^{0}}+\frac{\hat{v}_{-}^{0}-\breve{A}^{0} \hat{v}_{-}^{b}}{\zeta_{-} \eta_{-}^{0}}\right)\right. \\
& \left.-\frac{1}{2 r^{b}}\left(\hat{v}_{\zeta,+}^{b}-\hat{v}_{\zeta,-}^{b}+\frac{2 \bar{v}^{b}}{r^{b}}\left(\hat{v}_{+}^{b}+\hat{v}_{-}^{b}\right)\right)\right] .
\end{aligned}
$$

Using the formulae given at the end of this appendix, one can check explicitly that the above expressions are indeed $S U(2)$ invariant.

$\overline{26}$ Note that $d \zeta / f_{0+}^{2}=d \zeta^{[+]}$is the natural integration measure in the patch $\mathcal{U}_{+}$. 
Coordinates on the $\mathbb{C}^{2} / \mathbb{Z}_{2}$ fiber. The coordinates $\pi^{A^{\prime}}$ on the fiber of $\mathcal{S}$, (3.41) can be similarly generalized to the deformed case as follows:

$$
\begin{aligned}
\pi^{1} & =C \int_{C_{+}} \frac{\mathrm{d} \zeta}{2 \pi \mathrm{i}} \frac{\zeta}{f_{0+}^{2} \nu_{[+]}^{b}\left(f_{0+}^{2} \nu_{[+]}^{0}\right)^{1 / 2}} \\
& =\breve{\pi}^{1}\left[1-\frac{\hat{v}_{+}^{0}}{2 \zeta_{+} \eta_{+}^{0}}-\frac{\hat{v}_{\zeta,+}^{b}}{r^{b}}-\frac{\hat{v}_{+}^{b}}{2 r^{b}}\left(\frac{4 \bar{v}^{b}}{r^{b}}+\frac{x^{0}+2 v^{0} / \zeta_{+}}{\zeta_{+} \eta_{+}^{0}}\right)\right], \\
\pi^{2} & =-\bar{C} \int_{C_{-}} \frac{\mathrm{d} \zeta}{2 \pi \mathrm{i}} \frac{\zeta}{f_{0-}^{2} \nu_{[-]}^{b}\left(-f_{0-}^{2} \nu_{[-]}^{0}\right)^{1 / 2}} \\
& =\breve{\pi}^{2}\left[1-\frac{\hat{v}_{-}^{0}}{2 \zeta_{-} \eta_{-}^{0}}+\frac{\hat{v}_{\zeta,-}^{b}}{r^{b}}-\frac{\hat{v}_{-}^{b}}{2 r^{b}}\left(\frac{4 \bar{v}^{b}}{r^{b}}-\frac{x^{0}+2 v^{0} / \zeta_{-}}{\zeta_{-} \eta_{-}^{0}}\right)\right] .
\end{aligned}
$$

The conjugate variables $\bar{\pi}_{A^{\prime}}$ can be obtained from (C.5) using

$$
\overline{\zeta_{+}}=-1 / \zeta_{-}, \quad \overline{\eta_{+}^{\Lambda}}=\eta_{-}^{\Lambda}, \quad \overline{\hat{v}_{+}^{I}}=-\hat{v}_{-}^{I} / \zeta_{-}^{2}, \quad \overline{\hat{v}_{\zeta,+}^{I}}=-\hat{v}_{\zeta,-}^{I}+2 \hat{v}_{-}^{I} / \zeta_{-} .
$$

In particular, one has very simple relations

$$
\zeta_{+} \equiv-\frac{\pi^{1}}{\bar{\pi}_{2}}=\breve{\zeta}_{+}\left(1-\frac{\hat{v}_{+}^{b}}{r^{b} \zeta_{+}}\right), \quad \zeta_{-} \equiv \frac{\pi^{2}}{\bar{\pi}_{1}}=\breve{\zeta}_{-}\left(1+\frac{\hat{v}_{-}^{b}}{r^{b} \zeta_{-}}\right),
$$

whereas the variable $z=\pi^{1} / \pi^{2}$ parametrizing the fiber of $\mathcal{Z}_{\mathcal{M}}$ is given by

$$
\begin{aligned}
z=\breve{z} & {\left[1-\frac{\hat{v}_{+}^{0}}{2 \zeta_{+} \eta_{+}^{0}}+\frac{\hat{v}_{-}^{0}}{2 \zeta_{-} \eta_{-}^{0}}-\frac{\hat{v}_{\zeta,+}^{b}+\hat{v}_{\zeta,-}^{b}}{r^{b}}\right.} \\
& \left.-\frac{\hat{v}_{+}^{b}}{2 r^{b}}\left(\frac{4 \bar{v}^{b}}{r^{b}}+\frac{x^{0}+2 v^{0} / \zeta_{+}}{\zeta_{+} \eta_{+}^{0}}\right)+\frac{\hat{v}_{-}^{b}}{2 r^{b}}\left(\frac{4 \bar{v}^{b}}{r^{b}}-\frac{x^{0}+2 v^{0} / \zeta_{-}}{\zeta_{-} \eta_{-}^{0}}\right)\right] .
\end{aligned}
$$

To first order in the perturbation, the two quantities in (C.7) provide the zeros $\zeta_{ \pm}$of the deformed section $v^{b}$, and (2.57),(2.61) continue to hold. Using the explicit expressions (C.5) for $\pi^{A^{\prime}}$, one finds

$$
\frac{\mathrm{d} \mathbf{z}}{\mathbf{z}}=\left[\frac{r^{b}}{\zeta \eta^{b}}-\frac{1}{r^{b}}\left(\frac{\hat{v}_{+}^{b}}{\left(\zeta-\zeta_{+}\right)^{2}}+\frac{\hat{v}_{-}^{b}}{\left(\zeta-\zeta_{-}\right)^{2}}\right)\right] \mathrm{d} \zeta
$$

Relation to the contact geometric approach. Using this relation, one may relate the coordinates defined here to those defined in Sect. 5.2:

$$
\begin{aligned}
Y_{+}^{\Lambda} & =\mathcal{R} \mathcal{Z}^{\Lambda}\left(1-3 \mathrm{i} \sum_{j} \int_{\tilde{C}_{j}} \frac{\mathrm{d} \breve{\mathbf{z}}}{2 \pi \mathrm{i} \breve{\mathbf{z}}} \partial_{\rho_{b}} H_{(1)}^{[0 j]}(\breve{\xi}(\breve{\mathbf{z}}), \rho(\breve{\mathbf{z}}))\right) \\
A^{\Lambda} & =\mathcal{A}^{\Lambda}+\mathrm{i} \breve{\mathcal{R}} \sum_{j} \int_{\tilde{C}_{j}} \frac{\mathrm{d} \breve{\mathbf{z}}}{2 \pi \mathrm{i} \breve{\mathbf{z}}}\left(\breve{\mathbf{z}}^{-1} Z^{\Lambda}+\breve{\mathbf{z}} \bar{Z}^{\Lambda}\right) \partial_{\rho_{b}} H_{(1)}^{[0 j]}(\breve{\xi}(\breve{\mathbf{z}}), \rho(\breve{\mathbf{z}})), \\
r^{b} & =\frac{1}{2}\left(r_{+}^{b}+r_{-}^{b}\right), \quad B_{I}=\mathcal{B}_{I},
\end{aligned}
$$


where

$$
\begin{aligned}
\breve{\xi}^{\Lambda}(\breve{\mathbf{z}})= & \breve{A}^{\Lambda}+\breve{\mathcal{R}}\left(\breve{\mathbf{z}}^{-1} \breve{Z}^{\Lambda}-\breve{\mathbf{z}}^{\Lambda}\right) \\
\rho_{I}(\breve{\mathbf{z}})= & \breve{B}_{I}-\mathrm{i} \sum_{j} \int_{\tilde{C}_{j}} \frac{\mathrm{d} \breve{\mathbf{z}}^{\prime}}{2 \pi \mathrm{i} \breve{\mathbf{z}}^{\prime}} \frac{\breve{\mathbf{z}}^{\prime}+\breve{\mathbf{z}}}{\breve{\mathbf{z}}^{\prime}-\breve{\mathbf{z}}} H_{I}^{[i j]}\left(\breve{\xi}\left(\breve{\mathbf{z}}^{\prime}\right)\right) \\
& -\mathrm{i}\left(H_{I}^{[i 0]}(\breve{\xi}(\breve{\mathbf{z}}))+H_{I}^{[i \infty]}(\breve{\xi}(\breve{\mathbf{z}}))\right)-\mathrm{i} c_{I}^{[+-]} \log \breve{\mathbf{z}}
\end{aligned}
$$

parametrize the unperturbed twistor lines and $\breve{\mathbf{z}}$ is related to $\zeta$ through the (undeformed) relation $(2.61)$,

$$
\breve{\mathbf{z}}=-\frac{1}{\bar{z}} \frac{\zeta-\breve{\zeta}_{+}}{\zeta-\breve{\zeta}_{-}} .
$$

With these definitions and relations, it is tedious but straightforward to compute the deformed complex coordinate $\xi^{\Lambda}=u^{\Lambda} / u^{b}$ on $\mathcal{Z}$ in terms of the coordinates on $\mathcal{M} \times \mathbb{C} P^{1}$,

$$
\xi^{\Lambda}=A^{\Lambda}+z^{-1} Y_{+}^{\Lambda}-z Y_{-}^{\Lambda}+\mathrm{i} \sum_{j} \int_{\tilde{C}_{j}} \frac{\mathrm{d} \breve{\mathbf{z}}}{2 \pi \mathrm{i} \breve{\mathbf{z}}} \frac{\breve{\mathbf{z}}+z}{\breve{\mathbf{z}}-z}\left[\partial_{\rho_{\Lambda}} H_{(1)}^{[0 j]}-\breve{\xi}^{\Lambda}(\breve{\mathbf{z}}) \partial_{\rho_{b}} H_{(1)}^{[0 j]}\right] .
$$

Taking into account the relation

$$
\rho_{I}=-2 \mathrm{i} \breve{\xi}_{I}^{[i]}-\mathrm{i}\left(H_{I}^{[i 0]}(\breve{\xi})+H_{I}^{[i \infty]}(\breve{\xi})\right)
$$

one verifies that this expression coincides with the result (5.31) from contact geometry, at $\zeta=0, \mathbf{z}=z$. A similar derivation of $\tilde{\xi}_{I}^{[i]}$ in (5.39) ought to be possible but we have not attempted to carry it through.

Deformed hyperkähler potential. Having defined an appropriate set of coordinates on the Swann bundle, we now generalize the representation (3.56) for the hyperkähler potential to the deformed case, and relate it to the contact potential $\Phi_{[+]}$of contact geometry. For this purpose, we note that the hyperkähler potential (5.12) can be written as

$$
\begin{aligned}
\chi= & \left(1+\hat{v}_{0}^{I} \partial_{v^{I}}+\overline{\hat{v}}_{0}^{I} \partial_{\bar{v}^{I}}\right) \oint_{C} \frac{\mathrm{d} \zeta}{2 \pi \mathrm{i} \zeta}\left[1-x^{I}\left(\partial_{\eta^{I}}+\partial_{x^{I}} \rho_{J} \partial_{\rho_{J}}\right)\right] \\
& \times\left(H(\eta)+H_{(1)}(\eta, \rho)\right),
\end{aligned}
$$

where we omitted the summation over patches and used the relation (5.11) between the undeformed and deformed complex coordinates $v^{I}$ and $u^{I} \equiv v^{I}+\hat{v}_{0}^{I}$. Following the same steps (3.30) which led to the representation (3.56), one finds

$$
\begin{aligned}
& \chi=\left(1+\hat{v}_{0}^{I} \partial_{v^{I}}+\overline{\hat{v}}_{0}^{I} \partial_{\bar{v}^{I}}\right)\left[r^{\mathrm{b}} \breve{\mathcal{R}} \oint_{\tilde{C}} \frac{\mathrm{d} \breve{\mathbf{z}}}{2 \pi \mathrm{i} \breve{\mathbf{z}}}\left(\breve{\mathbf{z}}^{-1} \breve{Z}^{\Lambda}-\breve{\mathbf{z}} \breve{Z}^{\Lambda}\right)\left(H_{\Lambda}+H_{(1) \Lambda}\right)(\breve{\xi}(\breve{\mathbf{z}}), \rho(\breve{\mathbf{z}}))\right. \\
& \left.+r^{\mathrm{b}} c_{I}^{[+-]} \breve{A}^{I}\right]-\oint_{\tilde{C}} \frac{\mathrm{d} \zeta}{2 \pi \mathrm{i} \zeta}\left[\left(x^{I} \partial_{x^{I}}-\frac{v^{b} \zeta^{-1}+\bar{v}^{b} \zeta}{\eta^{b}} \zeta \partial_{\zeta}\right) \rho_{J}\right] \partial_{\rho_{J}} H_{(1)},
\end{aligned}
$$

where $\breve{\xi}^{\Lambda}(\breve{\mathbf{z}})$ and $\rho(\breve{\mathbf{z}})$ are given in (C.11). 
The unperturbed quantities $\breve{A}^{\Lambda}, \breve{Z}^{\Lambda}$, etc., are not $S U$ (2) invariant. To arrive at the desired form of the hyperkähler potential, we replace them in the first term of (C.16) by their deformed $\mathrm{SU}(2)$ invariant counterparts and collect the remaining terms which are all of order $\mathcal{O}\left(H_{(1)}\right)$. The first term in (C.16) then reads

$$
r^{\mathrm{b}} \mathcal{R} \oint_{\tilde{C}} \frac{\mathrm{d} \breve{\mathbf{z}}}{2 \pi \mathrm{i} \breve{\mathbf{z}}}\left(\breve{\mathbf{z}}^{-1} \mathcal{Z}^{\Lambda}-\breve{\mathbf{z}} \overline{\mathcal{Z}}^{\Lambda}\right)\left(H_{\Lambda}+H_{(1) \Lambda}\right)\left(\xi_{(0)}(\breve{\mathbf{z}}), \rho(\breve{\mathbf{z}})\right)+r^{\mathrm{b}} c_{I}^{[+-]} A^{I},
$$

where

$$
\xi_{(0)}^{\Lambda}(\breve{\mathbf{z}}) \equiv A^{\Lambda}+\breve{\mathbf{z}}^{-1} Y_{+}^{\Lambda}-\breve{\mathbf{z}} Y_{-}^{\Lambda} .
$$

The remaining terms are of three types: (i) the second term in (C.16), (ii) the terms coming from the derivatives with respect to $v^{I}$ and $\bar{v}^{I}$ in the first term in (C.16) and (iii) the terms coming from the difference between deformed and undeformed invariants in (C.17). Altogether they should combine in an invariant expression written as a contour integral of a $\mathcal{O}(-2)$ section. After a long calculation, one obtains

$$
\begin{aligned}
\chi= & r^{\mathrm{b}} \mathcal{R} \oint_{\tilde{C}} \frac{\mathrm{d} \breve{\mathbf{z}}}{2 \pi \mathrm{i} \breve{\mathbf{z}}}\left(\breve{\mathbf{z}}^{-1} \mathcal{Z}^{\Lambda}-\breve{\mathbf{z}} \overline{\mathcal{Z}}^{\Lambda}\right)\left(H_{\Lambda}^{[0 j]}+H_{(1) \Lambda}^{[0 j]}\right)+r^{\mathrm{b}} c_{I}^{[+-]} A^{I} \\
- & \mathrm{i} r^{\mathrm{b}} \mathcal{R} \oint_{\tilde{C}} \frac{\mathrm{d} \breve{\mathbf{z}}}{2 \pi \mathrm{i} \breve{\mathbf{z}}}\left(\breve{\mathbf{z}}^{-1} \mathcal{Z}^{\Sigma}-\breve{\mathbf{z}} \overline{\mathcal{Z}}^{\Sigma}\right)\left(H_{\Lambda \Sigma}^{[j 0]}+H_{\Lambda \Sigma}^{[j \infty]}\right) \\
& \left(\partial_{\rho_{\Lambda}} H_{(1)}^{[0 j]}-\xi_{(0)}^{\Lambda} \partial_{\rho_{b}} H_{(1)}^{[0 j]}\right) \\
+ & \mathrm{i} r^{\mathrm{b}} \mathcal{R} \oint_{\tilde{C}} \frac{\mathrm{d} \breve{\mathbf{z}}^{\prime}}{2 \pi \mathrm{i} \breve{\mathbf{z}}^{\prime}} \oint_{\tilde{C}} \frac{\mathrm{d} \breve{\mathbf{z}}}{2 \pi \mathrm{i} \breve{\mathbf{z}}} \frac{\breve{\mathbf{z}}^{\prime}+\breve{\mathbf{z}}}{\breve{\mathbf{z}}^{\prime}-\breve{\mathbf{z}}}\left(\breve{\mathbf{z}}^{-1} \mathcal{Z}^{\Sigma}-\breve{\mathbf{z}} \overline{\mathcal{Z}}^{\Sigma}\right) H_{\Lambda \Sigma}^{[i j]} \\
& \left.\left(\partial_{\rho_{\Lambda}} H_{(1)}^{[0 i]}-\xi_{(0)}^{\Lambda} \breve{\mathbf{z}}^{\prime}\right) \partial_{\rho_{b}} H_{(1)}^{[0 i]}\right),
\end{aligned}
$$

where $H_{\Lambda \Sigma}^{[i j]}$ are functions of $\xi_{(0)}(\breve{\mathbf{z}})$, whereas $H_{(1)}^{[0 i]}$ are functions of $\xi_{(0)}(\breve{\mathbf{z}})$ and $\rho(\breve{\mathbf{z}})$ (or, in the last term, functions of $\breve{\mathbf{z}}^{\prime}$ ).

This expression can be further simplified. First, taking into account (C.14) and

$$
H_{b}^{[i j]}(\xi)=\hat{H}^{[i j]}(\xi)-\xi^{\Lambda} \hat{H}_{\Lambda}^{[i j]}(\xi)+c_{\Lambda}^{[i j]} \xi^{\Lambda}+c_{b}^{[i j]},
$$

it is easy to check that the first and third terms in (C.19) combine into one with the derivatives of the transition functions replaced by $\tilde{T}_{\Lambda}^{[0 j]}\left(\xi_{(0)}, \tilde{\xi}^{[j]}\right)$ (5.23). Moreover, the last term in (C.19) can be rewritten as

$$
\begin{aligned}
& r^{\mathrm{b}} \mathcal{R} \sum_{j} \int_{\tilde{C}_{j}} \frac{\mathrm{d} \breve{\mathbf{z}}}{2 \pi \mathrm{i} \breve{\mathbf{z}}}\left(\breve{\mathbf{z}}^{-1} \mathcal{Z}^{\Sigma}-\breve{\mathbf{z}} \overline{\mathcal{Z}}^{\Sigma}\right) H_{\Lambda \Sigma}^{[0 j]} \\
& \times\left[-T_{[0 j]}^{\Lambda}+\frac{1}{2} \sum_{i} \int_{\tilde{C}_{i}} \frac{\mathrm{d} \breve{\mathbf{z}}^{\prime}}{2 \pi \mathrm{i} \breve{\mathbf{z}}^{\prime}} \frac{\breve{\mathbf{z}}^{\prime}+\breve{\mathbf{z}}}{\breve{\mathbf{z}}^{\prime}-\breve{\mathbf{z}}} T_{[0 i]}^{\Lambda}\right],
\end{aligned}
$$

where for $i=j$ the variable $\breve{\mathbf{z}}^{\prime}$ lies inside the contour for $\breve{\mathbf{z}}$ and the first term appears since in (C.19) the situation was opposite. Then the expression in the square brackets is just $\xi_{[0]}^{\Lambda}(\breve{\mathbf{z}})-\xi_{(0)}^{\Lambda}(\breve{\mathbf{z}})$ for $\breve{\mathbf{z}} \in \mathcal{U}_{j}$. Finally, $Y^{\Lambda}$ and $\mathcal{R} \mathcal{Z}^{\Lambda}$ differ by a phase factor (see 
(C.10)) which can be absorbed into a redefinition of the integration variable $\breve{\mathbf{z}}$. As a result, the hyperkähler potential can be written more compactly as

$$
\chi=r^{\mathrm{b}} \sum_{j} \oint_{\tilde{C}_{j}} \frac{\mathrm{d} \breve{\mathbf{z}}}{2 \pi \mathrm{i} \breve{\mathbf{z}}}\left(\breve{\mathbf{z}}^{-1} Y_{+}^{\Lambda}-\breve{\mathbf{z}} Y_{-}^{\Lambda}\right) \tilde{T}_{\Lambda}^{[0 j]}\left(\xi_{[0]}, \tilde{\xi}^{[j]}\right)+r^{\mathrm{b}} c_{I}^{[+-]} A^{I}
$$

Comparing with the contact potential (5.45), one finds that the relation (3.38) continues to hold in the perturbed case.

Deformed $S U$ (2) transformations. The $S U(2)$ invariance of the quantities defined in this appendix can be checked using the following transformation rules, which follow from the general discussion in Appendix A:

$$
\begin{aligned}
& \delta v^{I}=\mathrm{i} \epsilon_{3} v^{I}+\epsilon_{+} x^{I}-\frac{3}{2} \epsilon_{-} \overline{\hat{v}}_{3}^{I}, \quad \delta \bar{v}^{I}=-\mathrm{i} \epsilon_{3} \bar{v}^{I}+\epsilon_{-} x^{I}-\frac{3}{2} \epsilon_{+} \hat{v}_{3}^{I}, \\
& \delta x^{I}=-2\left(\epsilon_{-} v^{I}+\epsilon_{+} \bar{v}^{I}\right), \quad \delta \varrho_{I}=\mathrm{i}\left(\epsilon_{-} \mathcal{L}_{\bar{u}^{I}}-\epsilon_{+} \mathcal{L}_{u^{I}}\right)+\epsilon_{3} c_{I}, \\
& \delta w_{I}=\epsilon_{+} \mathcal{L}_{u^{I}}+\frac{\mathrm{i}}{2} \epsilon_{3} c_{I}, \quad \delta \bar{w}_{I}=\epsilon_{-} \mathcal{L}_{\bar{u}^{I}}-\frac{\mathrm{i}}{2} \epsilon_{3} c_{I}, \\
& \delta \hat{v}_{0}^{I}=\mathrm{i} \epsilon_{3} \hat{v}_{0}^{I}+i \epsilon_{+} \mathcal{L}_{\varrho_{I}}+\frac{3}{2} \epsilon_{-} \overline{\hat{v}}_{3}^{I}, \\
& \delta \hat{v}_{ \pm}^{I}=\left(\mathrm{i} \epsilon_{3}-2 \epsilon_{-} \zeta_{ \pm}\right) \hat{v}_{ \pm}^{I}-\frac{3}{2}\left(\epsilon_{+} \zeta_{ \pm}^{2} \hat{v}_{3}^{I}-\epsilon_{-} \overline{\hat{v}}_{3}^{I}\right), \\
& \delta \hat{v}_{\zeta, \pm}^{I}=-2 \epsilon_{-} \hat{v}_{ \pm}^{I}-3 \epsilon_{+} \zeta_{ \pm} \hat{v}_{3}^{I}, \\
& \delta \hat{v}_{\zeta \zeta, \pm}^{I}=-\left(i \epsilon_{3}-2 \epsilon_{-} \zeta_{ \pm}\right) \hat{v}_{\zeta \zeta, \pm}^{I}-2 \epsilon_{-} \hat{v}_{\zeta, \pm}^{I}-3 \epsilon_{+} \hat{v}_{3}^{I},
\end{aligned}
$$

where

$$
\hat{v}_{0}^{I}=\oint_{C} \frac{\mathrm{d} \zeta}{2 \pi} H_{(1)}^{I}, \quad \hat{v}_{3}^{I}=\oint_{C} \frac{\mathrm{d} \zeta}{\pi \zeta^{3}} H_{(1)}^{I}, \quad \overline{\hat{v}}_{3}^{I}=-\oint_{C} \frac{\mathrm{d} \zeta}{\pi \zeta^{-1}} H_{(1)}^{I}
$$

are Laurent coefficients of the deformation $\hat{v}_{0}^{I}$ given in (5.7) (as usual, we omitted the sum over contours).

The following properties, valid in the absence of perturbations, are also useful:

$$
\begin{aligned}
\delta \zeta_{ \pm} & =-\epsilon_{+}-\epsilon_{-} \zeta_{ \pm}^{2}+\mathrm{i} \epsilon_{3} \zeta_{ \pm}, \\
\delta \eta_{ \pm}^{\Lambda} & =-\left(\epsilon_{+} \bar{\zeta}_{\mp}+\epsilon_{-} \zeta_{ \pm}\right) \eta_{ \pm}^{\Lambda}, \\
\delta z & =\frac{1+z \bar{z}}{|z|} \sqrt{\frac{\bar{v}^{b}}{v^{b}}} z \epsilon_{+}, \quad \delta \bar{z}=\frac{1+z \bar{z}}{|z|} \sqrt{\frac{v^{b}}{\bar{v}^{b}}} \bar{z} \epsilon_{-}, \\
\delta \rho_{I} & =\left(\epsilon_{+}+\epsilon_{-} \zeta^{2}-\mathrm{i} \epsilon_{3} \zeta\right) \partial_{\zeta} \rho_{I} .
\end{aligned}
$$




\section{References}

1. Alexandrov, S., Pioline, B., Saueressig, F., Vandoren, S.: Linear perturbations of Hyperkähler metrics. Lett. Math. Phys. 87, 225 (2009)

2. Swann, A.: Hyper-Kähler and quaternionic Kähler geometry. Math. Ann. 289(3), 421-450 (1991)

3. de Wit, B., Kleijn, B., Vandoren, S.: Superconformal hypermultiplets. Nucl. Phys. B568, 475-502 (2000)

4. de Wit, B., Roček, M., Vandoren, S.: Hypermultiplets, hyperkähler cones and quaternion-Kähler geometry. JHEP 02, 039 (2001)

5. Bergshoeff, E.A., Cucu, S., de Wit, T., Gheerardyn, J., Van Proeyen, A., Vandoren, S.: The map between conformal hypercomplex/hyper-Kähler and quaternionic(-Kähler) geometry. Commun. Math. Phys. 262, 411-457 (2006)

6. de Wit, B., Roček, M., Vandoren, S.: Gauging isometries on hyperkaehler cones and quaternion- kaehler manifolds. Phys. Lett. B511, 302-310 (2001)

7. Ketov, S.V.: Superconformal hypermultiplets in superspace. Nucl. Phys. B582, 95-118 (2000)

8. Kuzenko, S.M.: On superconformal projective hypermultiplets. JHEP 12, 010 (2007)

9. Kuzenko, S.M., Lindström, U., Roček, M., Tartaglino-Mazzucchelli, G.: 4D $\mathcal{N}=2$ Supergravity and Projective Superspace. JHEP 0809, 051 (2008)

10. de Wit, B., Saueressig, F.: Off-shell $\mathcal{N}=2$ tensor supermultiplets. JHEP 09, 062 (2006)

11. de Wit, B., Saueressig, F.: Tensor supermultiplets and toric quaternion-Kaehler geometry. Fortsch. Phys. 55, 699-704 (2007)

12. Salamon, S.M.: Quaternionic Kähler manifolds. Invent. Math. 67(1), 143-171 (1982)

13. LeBrun, C.: Quaternionic-Kähler manifolds and conformal geometry. Math. Ann. 284(3), 353-376 (1989)

14. LeBrun, C.: Fano manifolds, contact structures, and quaternionic geometry. Internat. J. Math. 6(3), 419-437 (1995)

15. LeBrun, C.: A Rigidity Theorem for Quaternionic-Kahler Manifolds. Proc. Amer. Math. Soc. 103(4), 1205-1208 (1988)

16. LeBrun, C., Salamon, S.: Strong rigidity of positive quaternion-Kähler manifolds. Invent. Math. 118(1), 109-132 (1994)

17. Neitzke, A., Pioline, B., Vandoren, S.: Twistors and black holes. JHEP 04, 038 (2007)

18. Bagger, J., Witten, E.: Matter couplings in $\mathcal{N}=2$ supergravity. Nucl. Phys. B222, 1 (1983)

19. Alexandrov, S., Pioline, B., Saueressig, F., Vandoren, S.: D-instantons and twistors. JHEP 0903, 044 (2009)

20. Boyer, C.P., Galicki, K.: 3-Sasakian manifolds. Surv. Diff. Geom. 7, 123-184 (1999)

21. Atiyah, M.F., Hitchin, N.J., Singer, I.M.: Self-duality in four-dimensional Riemannian geometry. Proc. Roy. Soc. London Ser. A 362(1711), 425-461 (1978)

22. Hitchin, N.J., Karlhede, A., Lindström, U., Roček, M.: Hyperkähler metrics and supersymmetry. Commun. Math. Phys. 108, 535 (1987)

23. Hitchin, N.: The self-duality equations on a Riemann surface. Proc. London Math. Soc. (3) 55(1), 59$126(1987)$

24. Ivanov, I.T., Roček, M.: Supersymmetric sigma models, twistors, and the Atiyah-Hitchin metric. Commun. Math. Phys. 182, 291-302 (1996)

25. Lindström, U., Roček, M.: Properties of Hyperkähler manifolds and their twistor spaces. Commun. Math. Phys. 293, 257-278 (2010)

26. Galicki, K.: A generalization of the momentum mapping construction for quaternionic Kähler manifolds. Commun. Math. Phys. 108(1), 117-138 (1987)

27. Neitzke, A.: Private communication

28. Alexandrov, S.: Quantum covariant c-map. JHEP 05, 094 (2007)

29. Cecotti, S., Ferrara, S., Girardello, L.: Geometry of type II superstrings and the moduli of superconformal field theories. Int. J. Mod. Phys. A4, 2475 (1989)

30. Ferrara, S., Sabharwal, S.: Quaternionic manifolds for type II superstring vacua of Calabi-Yau spaces. Nucl. Phys. B332, 317 (1990)

31. de Wit, B., Van Proeyen, A.: Potentials and symmetries of general gauged $\mathcal{N}=2$ supergravity: Yang-Mills models. Nucl. Phys. B245, 89 (1984)

32. Cremmer, E., de Wit, B., Derendinger, J.P., Ferrara, S., Girardello, L., Kounnas, C., Van Proeyen, A.: Vector multiplets coupled to $\mathcal{N}=2$ supergravity: SuperHiggs effect, flat potentials and geometric structure. Nucl. Phys. B250, 385 (1985)

33. Roček, M., Vafa, C., Vandoren, S.: Hypermultiplets and topological strings. JHEP 02, 062 (2006)

34. Roček, M., Vafa, C., Vandoren, S.: Quaternion-Kähler spaces, hyperkähler cones, and the c-map. http:// arXiv.org/abs/math/0603048v3[math.DG], 2006

35. Antoniadis, I., Ferrara, S., Minasian, R., Narain, K.S.: $R^{4}$ couplings in M- and type II theories on Calabi-Yau spaces. Nucl. Phys. B507, 571-588 (1997) 
36. Robles-Llana, D., Saueressig, F., Vandoren, S.: String loop corrected hypermultiplet moduli spaces. JHEP 03, 081 (2006)

37. Antoniadis, I., Minasian, R., Theisen, S., Vanhove, P.: String loop corrections to the universal hypermultiplet. Class. Quant. Grav. 20, 5079-5102 (2003)

38. Günther, H., Herrmann, C., Louis, J.: Quantum corrections in the hypermultiplet moduli space. Fortsch. Phys. 48, 119-123 (2000)

39. Ionas, R.A.: Elliptic constructions of Hyperkähler metrics II: The quantum mechanics of a Swann bundle. http://arXiv.org/abs/0712.3600v1[math.DG], 2007

Communicated by N.A. Nekrasov 